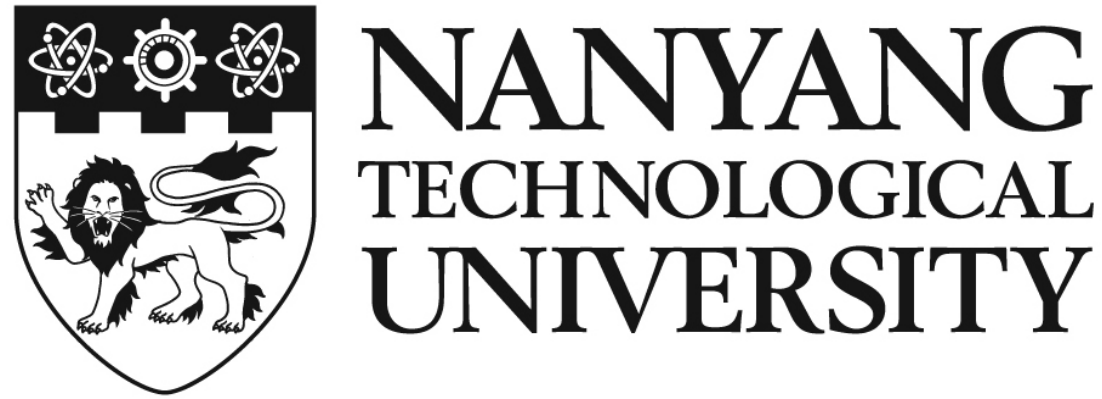

\title{
ON-LINE HUMAN ACTIVITY RECOGNITION IN VIDEO STREAMS
}

I
Ð
O

HU BO

$\stackrel{N}{\circ}$

SCHOOL OF ELECTRICAL \& ELECTRONIC

ENGINEERING

2017 



\section{ON-LINE HUMAN ACTIVITY RECOGNITION IN VIDEO STREAMS}

I
망

HU BO

School of Electrical \& Electronic Engineering

A thesis submitted to the Nanyang Technological University in partial fulfilment of the requirement for the degree of Master of Engineering 



\section{Acknowledgments}

Firstly, I would like to express my most sincere gratitude to my supervisor, Professor Junsong Yuan for his full support, patient instruction, and encouragement. He guided me into the attractive research realm of computer vision and machine learning. His open-mindedness and conscientiousness make a profound impact on my research work. I would also like to express my special appreciation to my former supervisor Professor Hong Cheng from University of Electronic Science and Technology of China (UESTC). It was his warm recommendation that provided me with the precious opportunity for becoming a master student under Professor Junsong Yuan's supervision in Nanyang Technological University (NTU).

Besides, my gratefulness should also go to all of the excellent members in my research group. Their brilliant thoughts inspired me a lot on the research and encourage me to face the challenges. I would like to thank my family, my friends and my beloved girlfriend. Life is enjoyable with your company.

Lastly, a sincere the School of Electrical \& Electronic Engineering, NTU, for providing me with the nice environment and equipment for my research work. 



\section{Contents}

Acknowledgments $\quad$ i

Contents

Summary vii

$\begin{array}{ll}\text { List of abbreviations ix } & \text { ix }\end{array}$

List of tables $\quad$ xi

List of figures $\quad$ xiii

1 Introduction $\quad 1$

1.1 Background .......................... 1

1.2 Motivation .......................... 2

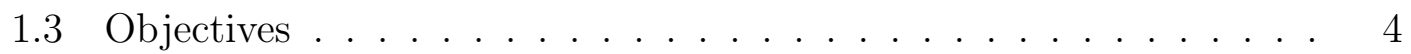

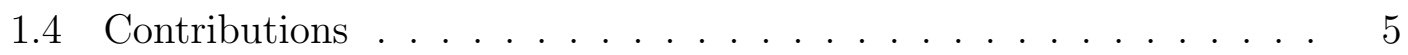

1.5 Organization of the Report . . . . . . . . . . . . . . . 6

2 Literature Review $\quad 9$

2.1 Low-level Features . . . . . . . . . . . . . . . . . . . . . . . 9

2.2 Middle-level Representation . . . . . . . . . . . . . . . . 11

2.3 Off-line Human Action Recognition . . . . . . . . . . . . . . 13 
2.4 On-line Human Action Recognition . . . . . . . . . . . . . . . 15

2.5 Human Action Prediction . . . . . . . . . . . . . . . . . . 16

2.6 Human Activity Recognition . . . . . . . . . . . . . . . . . . . . . . 17

2.7 Anomaly Detection and Alarm Generation . . . . . . . . . . . . 18

3 On-line Action Recognition $\quad 21$

3.1 Introduction . . . . . . . . . . . . . . . . . . . 21

3.2 Multiple Assignments for BoW Representation . . . . . . . . . . . . 23

3.2 .1 Model . . . . . . . . . . . . . . . . . . . . 23

3.2.2 Toy Problem . . . . . . . . . . . . . . . . . . . . . . 24

3.3 Discriminative Action States Discovery . . . . . . . . . . . . . 27

3.3.1 Notations ...................... . . 27

3.3.2 Discover Single Action State . . . . . . . . . . . . . . . 27

3.3.3 Discover Multiple Action States . . . . . . . . . . . . . . 30

3.3.4 Testing Stage . . . . . . . . . . . . . . . . . . . 31

3.3 .5 Optimization . . . . . . . . . . . . . . 33

3.4 Experiments . . . . . . . . . . . . . . . . . 35

3.4.1 Experiment settings . . . . . . . . . . . 36

3.4 .2 On-line Human Action Recognition . . . . . . . . . . . . . 38

3.4.3 Human Action Prediction . . . . . . . . . . . . . . . 43

3.5 Real-time Implementation . . . . . . . . . . . . . . . . . . . . . 45

3.6 Concluding Remarks . . . . . . . . . . . . . . . . . . . . . . 47

4 On-line Activity Recognition and Anomaly Detection 49

4.1 Introduction . . . . . . . . . . . . . . . . . . . . . . . 49

4.2 Statistical Activity Model . . . . . . . . . . . . . . . . . 51

4.2 .1 Notations . . . . . . . . . . . . . . . . 51 
4.2.2 Joint Activity Recognition and Alarm Generation . . . . . . 51

4.2.3 Parameters Estimation . . . . . . . . . . . . . . . 60

4.2 .4 Inference . . . . . . . . . . . . . . . . . 62

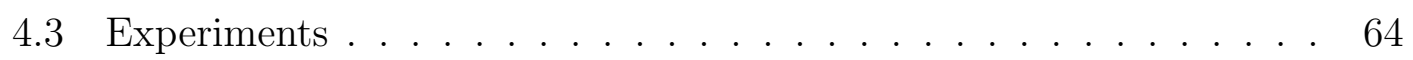

4.3 .1 Settings . . . . . . . . . . . . . . . . 64

4.3.2 Experiment on Action State Segmentation . . . . . . . . . 67

4.3.3 Experiment on Activity Recognition . . . . . . . . . . . . 69

4.3.4 Experiment on Alarm Generation . . . . . . . . . . . 70

4.4 Concluding Remarks . . . . . . . . . . . . . . . . . . 72

5 Conclusions and Future Work $\quad 73$

5.1 Conclusions . . . . . . . . . . . . . . . . 73

5.2 Future Work ......................... 74

5.2.1 Real-world Testing ................ . . 75

5.2.2 Adaptive Action States Discovery . . . . . . . . . . . 75

5.2.3 On-line Multi-person Action and Activity Recognition . . . 75

$\begin{array}{ll}\text { List of publications } & 77\end{array}$

$\begin{array}{lr}\text { Bibliography } & 79\end{array}$ 



\section{Summary}

Human action and activity recognition have been playing an important role in computer vision. On-line action and activity recognition, which is defined as recognizing ongoing actions or activities in untrimmed videos, is a necessity of a real-time recognition system that can be used in many real world scenarios such as abnormal action detection by video surveillance and robotics. Despite the progress made in recent years, on-line action and activity recognition at a real-time speed still remains a challenging problem. In the task of on-line action recognition, decisions are made based on partial observation of the actions. Thus the testing video is represented by a sequence of frame-level descriptors. Due to the fact that local features from a small part of the video are not sufficient to generate frame-level descriptors, these frame-level descriptors may become noisier. Besides, frame-level descriptors from one action category contain large intra-class variation since the appearance and motion of an action are varying temporally and each of these descriptors comes from a small part of the action called action state. Human activities, such as cooking a dish, are much longer and more complex than actions. On-line activity recognition is more difficult since the positions of action states of an activity are not provided. Hence, the method of on-line activity recognition should parse the action states and activities in the testing video and recognize them at the same time. Another challenge is that shared action states in multiple activity categories may heavily weaken the recognition confidence and result in incorrect activity classification. These challenges make on-line activity recognition 
a difficult problem.

Motivated by the above observations, in this thesis, we present a systematic study to address these challenging problems. Firstly, the standard Bag-of-Words (BoW) representation is improved by assigning each local feature vector to multiple nearest neighbor codes in the codebook to suppress the noise. Then we introduce a Discriminative Action States Discovery (DASD) method for on-line action recognition. In the proposed approach, the positive sample set is treated as multiple patterns called action states. To solve the problem caused by the large intra-class variation, DASD method discovers different distributions of frame-level descriptors from each action category while training classifiers for the action states. The action state models are effectively learned by clustering the positive samples and optimizing the decision boundary of each state simultaneously. Lastly, a Statistical Activity Model (SAM) is proposed for the task of on-line activity recognition and anomaly detection. This model employs DASD algorithm to parse the positions of action states in the untrimmed testing video and captures temporal dependencies between action states to recognize human activities. Furthermore, alarms are generated when the action states are incorrect according to the activity. The on-line inference of SAM is solved by on-line dynamic programming.

The proposed DASD model is evaluated on the tasks of on-line action recognition and action prediction. Experimental results show that our methods not only outperform the baseline methods and state-of-the-art methods for on-line action recognition. The SAM algorithm is evaluated by the experiments on on-line action state segmentation, activity recognition, and anomaly detection, where our method achieve the best performance. Last but not least, the proposed methods recognize actions and activities in an on-line processing manner with the real-time speed. 


\title{
List of Abbreviations
}

\author{
BoW Bag of Words \\ CRF Conditional Random Field \\ CNN Convolution Neural Network \\ C3D 3D Convolution Neural Network \\ DBN Dynamic Bayesian Network \\ DTF Dense Trajectory Feature \\ DASD Discriminative Action State Discovery \\ GMM Gaussian Mixture Model \\ HCI Human Computer Interaction \\ HMM Hidden Markov Model \\ HoG Histogram of Gradient \\ HoF Histogram of Optical Flow \\ ITF Improved Trajectory Feature \\ IoU Intersection over Union \\ k-NN $\quad$ k Nearest Neighbor \\ LOO Leave-One-Out \\ MEMM Maximum-Entropy Markov Model \\ MBH Motion Boundary Histogram \\ NN Nearest Neighbor \\ RBF Radial Based Function \\ SAM Statistical Activity Model \\ STIP Spatial-Temporal Interest Point \\ SVM Support Vector Machine \\ VLAD Vector of Locally Aggregated Descriptors
}





\section{List of Tables}

2.1 Comparison of BoW, VLAD, and Fisher Vector. . . . . . . . . . . 12

2.2 Comparison of the task of off-line action recognition, on-line action recognition, and action prediction. . . . . . . . . . . . 16

3.1 Results of Multi-assignment BoW on privately collected dataset . . 38

3.2 Names of 20-class in the subset of UCF-101. . . . . . . . . . . . . 40

3.3 Experiment results on different features employed by our model. . . 41

3.4 Comparison of time cost (ms) of different descriptors and classifiers. 46

3.5 Speed for every step in the testing stage. . . . . . . . . . . . . . . 46

4.1 Warm up activities and the contained action states in WarmUp2 dataset . . . . . . . . . . . . . . . . . 6 64

4.2 Average IoU of our approach and baselines in the experiment on action states segmentation. . . . . . . . . . . . . 67

4.3 Mean accuracy of our approach and baselines in the experiment on activity recognition. . . . . . . . . . . . . . . . 69

4.4 Average precision of 3 types of alarms generation. . . . . . . . 71 



\section{List of Figures}

1.1 The comparison between off-line action recognition and on-line action recognition. . . . . . . . . . . . . . . . 3

1.2 The structure of this thesis. . . . . . . . . . . . . . 6

2.1 C3D network architecture $[1] . \ldots \ldots \ldots$

2.2 The diagram of off-line action recognition. . . . . . . . . . . . . 14

2.3 The diagram of on-line action recognition. . . . . . . . . . . 15

2.4 Co-occurrence graph in $[2] \ldots \ldots \ldots \ldots$. . . . . . . . . . . . . 17

2.5 Illustration of the principle of alarm generation. $\ldots \ldots . . \ldots 18$

3.1 Illustration of the DASD. . . . . . . . . . . . . . . 22

3.2 Illustration of Multi-assignment BoW representation. . . . . . . . . 23

3.3 Results on the toy problem. . . . . . . . . . . . 26

3.4 Illustration for the ignoring mechanism. . . . . . . . . . . . . . 29

3.5 Framework of the proposed on-line human action recognition system based on DASD. . . . . . . . . . . . . . . . . . . 32

3.6 Some examples of our privately collected dataset. . . . . . . . . . . 37

3.7 Accuracy for different numbers of action states $K$ on privately collect dataset. . . . . . . . . . . . . . . . . . 39

3.8 Some examples of the result on privately collect dataset. . . . . . . 40

3.9 Accuracy of the 20-class of UCF-101. . . . . . . . . . . . . 41 
3.10 Accuracy for different numbers of action states $K$ on UCF-101. . . 42

3.11 Confusion matrices of [3] (left) and Discriminative Action States Discovery (right). . . . . . . . . . . . . . . . 43

3.12 Results of recognition accuracy based on different observation ratio on UT-Interaction dataset. . . . . . . . . . . . . . . . . . . . . . . 45

4.1 The diagram of joint action state segmentation, activity recognition, and alarm generation. . . . . . . . . . . . . . . 50 50

4.2 Comparison between graphs w/o loops. Left: graph without loops; Right: graph with loop $2 \rightarrow 3 \rightarrow 2$. . . . . . . . . . . 58

4.3 Illustration of the Statistical Activity Model from the perspective of graphic model. . . . . . . . . . . . . . . . 60

4.4 Some frame examples of WarmUp2 dataset. . . . . . . . . . . . . 65

4.5 Average IoU in activity categories of our approach and baselines. . . 67

4.6 An example of the output probabilities of action states by different methods. . . . . . . . . . . . . . . . . . . 68

4.7 Recognition accuracy in activity categories of our approach and the baselines. . . . . . . . . . . . . . . . . . 70

4.8 The curve of precision and recall on alarm generation for abnormal action states by different methods. . . . . . . . . . . . . 71 


\section{Chapter 1}

\section{Introduction}

\subsection{Background}

Over the last decade, with the development of camera technology and cameraequipped mobile devices, recognizing human actions and activities in videos are of great importance for automatic analysis of the huge amount of videos. Although researchers have achieved great progress on the task of human action and activity recognition, most of the state-of-the-art approaches recognize actions or activities through the off-line process: given a trimmed video that contains one action or activity, determine which class it belongs to based on the full observation of the video. Different from the off-line action and activity recognition, on-line recognition is defined as recognizing ongoing actions or activities in an untrimmed video, which means that the labels should be given when the video is still playing. The details of the difference of off-line recognition and on-line recognition will be explained in Section 2.5.

On-line action recognition algorithm is a necessity of the real-time action recognition system that can be used in many real world scenarios. For example, in the video surveillance system, it is helpful to detect the abnormal actions in a public 
place in time, such as leaving a suspicious bag on the floor $[4,5]$. For a health care system, detecting the action of falling down or other dangers of a senior person at home enables a timely rescue. In interactive video games, which is based on Human Computer Interaction (HCI) technology [6], on-line action recognition allows the real-time feedback to the players and enhances the play experience. Differently, activities are more complex than actions and usually consist of a sequence of action states with a flexible order. For example, in the activity of making a cup of milk tea with the tea bag, there are three main action states: put the tea bag into the cup, pour the hot water into the cup, and pour the milk into the cup. The order of the three action states is flexible but not random: one may pour the milk before the hot water, however, putting in the tea bag should be the first state. On-line activity recognition is useful for the intelligent assistant system. For instance, recognizing the activities of manufacture will help the inspectors to check if the product is assembled in a correct way. Similarly, on-line activity recognition can also supervise the training of soldiers or assist people to do workout or cook. Despite many previous works, efficient and robust on-line action and activity recognition still remain challenging problems.

\subsection{Motivation}

For the off-line action or activity recognition task, many works show good performance. Despite the success of these methods, the extension from off-line recognition to on-line recognition is not straightforward. The main reason is that in online recognition, the decisions are made based on partial observation of the testing video. To represent a video in off-line action recognition, video-level descriptors are computed based on local feature vectors of the whole video. Different from off-line action recognition, in the on-line action recognition task, a video is usually represented by a series of frame-level descriptors where each descriptor corresponds to 


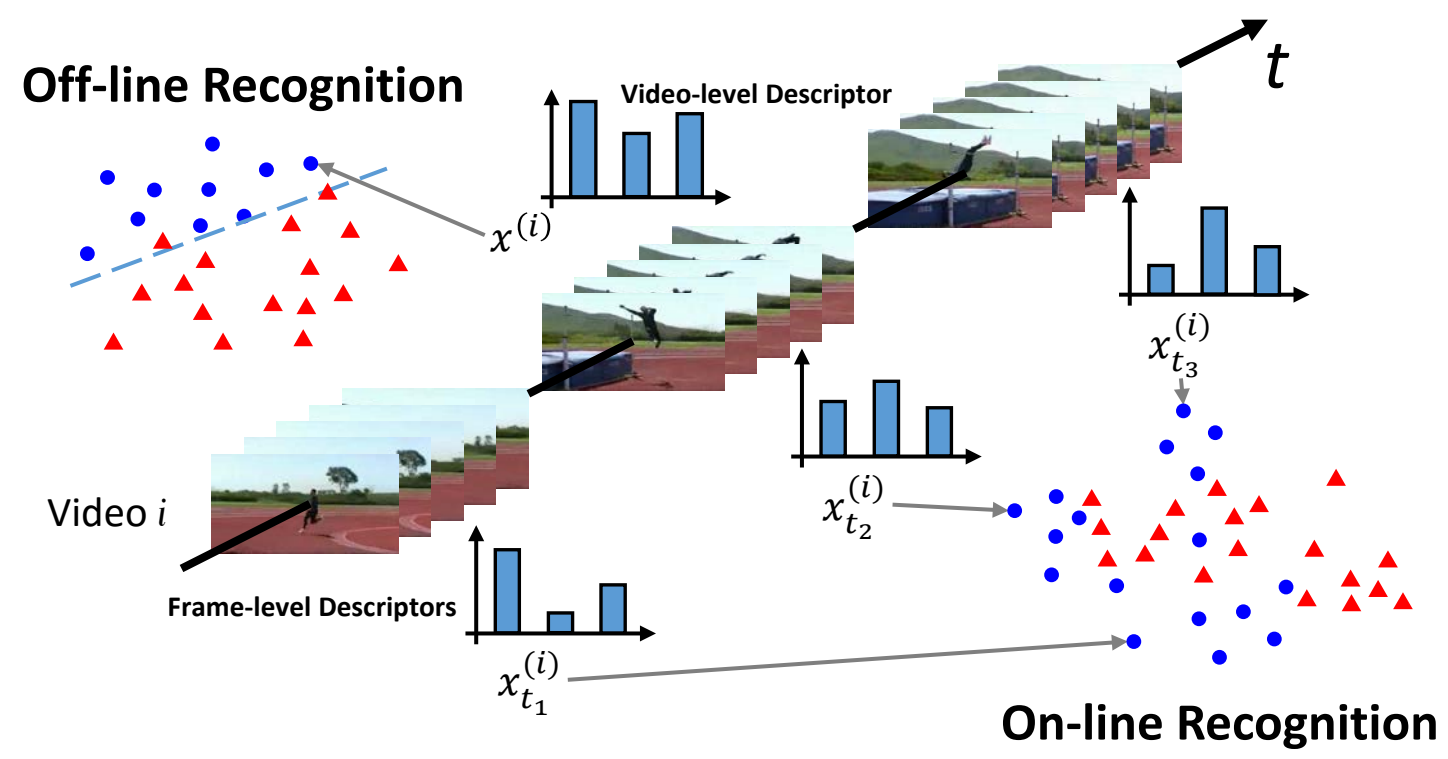

Figure 1.1: The comparison between off-line action recognition and on-line action recognition.

one frame or several frames in a temporal interval of the video. Frame-level descriptors may contain more noise than video-level descriptors since there are insufficient local feature vectors provided for quantization, which results in a set of very sparse frame-level descriptors. Furthermore, because both the appearance and motion of an action are varying temporally, frame-level descriptors derived from the same action class may obey different distributions. For example, the action "high jump" in Figure. 1.1 roughly consists of three different action states, "approach (run)" "jump" and "land back on ground". Frame-level descriptors of one action category, which are represented by blue circles, integrate the information from one of the three action states respectively. Thus, these frame-level descriptors follow different distributions in the descriptor space and intra-class variation is larger, where it is impossible to separate the positive samples from negative samples (red triangles in Figure. 1.1) by a linear decision boundary.

Different from action, human activity is usually much longer and more complex, such as a whole program of workout or cooking a dish. Activities also consist of different action states, of which the order is more evident and more strict. In 
the task of activity recognition, the main challenges come from that the positions of action states and activities are unknown and there are shared action states in different activity categories which may probably confuse the classifiers. Hence two main problems are required to be solved. The first is the action state parsing and temporal segmentation, which tells what the action states are and where they are. The second is activity recognition based on the segmentation result and the temporal dependencies between action states. Besides, we aim to generate the alarms for abnormal action states in an activity at the same time of activity recognition. However, training samples with abnormal actions are not provided. Therefore, the algorithm requires an alarm generation mechanism.

In short, in this thesis, we will focus on modeling the actions and activities in on-line recognition scenario and solving the challenges mentioned above.

\section{$1.3 \quad$ Objectives}

There are two major purposes of this thesis.

Fist of all, based on the observation mentioned in Section 1.2, a novel approach of on-line action recognition need to be designed which overcome the problem caused by larger intra-class variation and noise. Since real-time speed is highly demanded in many applications, the efficiency of the algorithm is also considered.

Secondly, we aim to propose an algorithm for joint on-line activity recognition and anomaly detection in untrimmed video based on the previous action recognition method, which needs to solve the problems caused by shared action states in the activities and the unavailability of the positions of action states and activities.

Overall, the objectives of this work are listed as follows:

- propose an algorithm for on-line human action recognition and action prediction; 
- build a real-time action recognition system based on the proposed method;

- propose an on-line activity approach which is able to send alarms for abnormal actions at the same time.

\subsection{Contributions}

To address the challenges in on-line human action and activity recognition problems, a systematic work has been conducted in this thesis.

In order to solve the problem caused by large intra-class variation in on-line action recognition, a multiple assignment of Bag-of-Words (BoW) is introduced, which assigns each local feature vector to multiple nearest neighbor codes in the codebook. Furthermore, a Discriminative Action States Discovery (DASD) method is proposed. In most of the existing algorithm, the descriptors from an action are usually treated as a whole pattern when training the classifier such as Support Vector Machine (SVM). Instead, our proposed model is good at discovering different states of an action and training a classifier for each action state of the action simultaneously. Based on the proposed algorithm, an on-line action recognition system is built, which not only shows good performance, but also recognizes actions in real-time.

In the task of on-line activity recognition, a statistical activity model with novel alarm generation mechanism is proposed. This approach is able to jointly solve the problem of action state segmentation, activity recognition, and alarm generation. To solve the problem of unavailability of the positions of action states and activities and the confusion caused by shared action states in different activity categories, the temporal dependencies between action states are captured. Thus, the action state segmentation and activity recognition results are obtained based on the whole observed action state sequence instead of the current state only. 


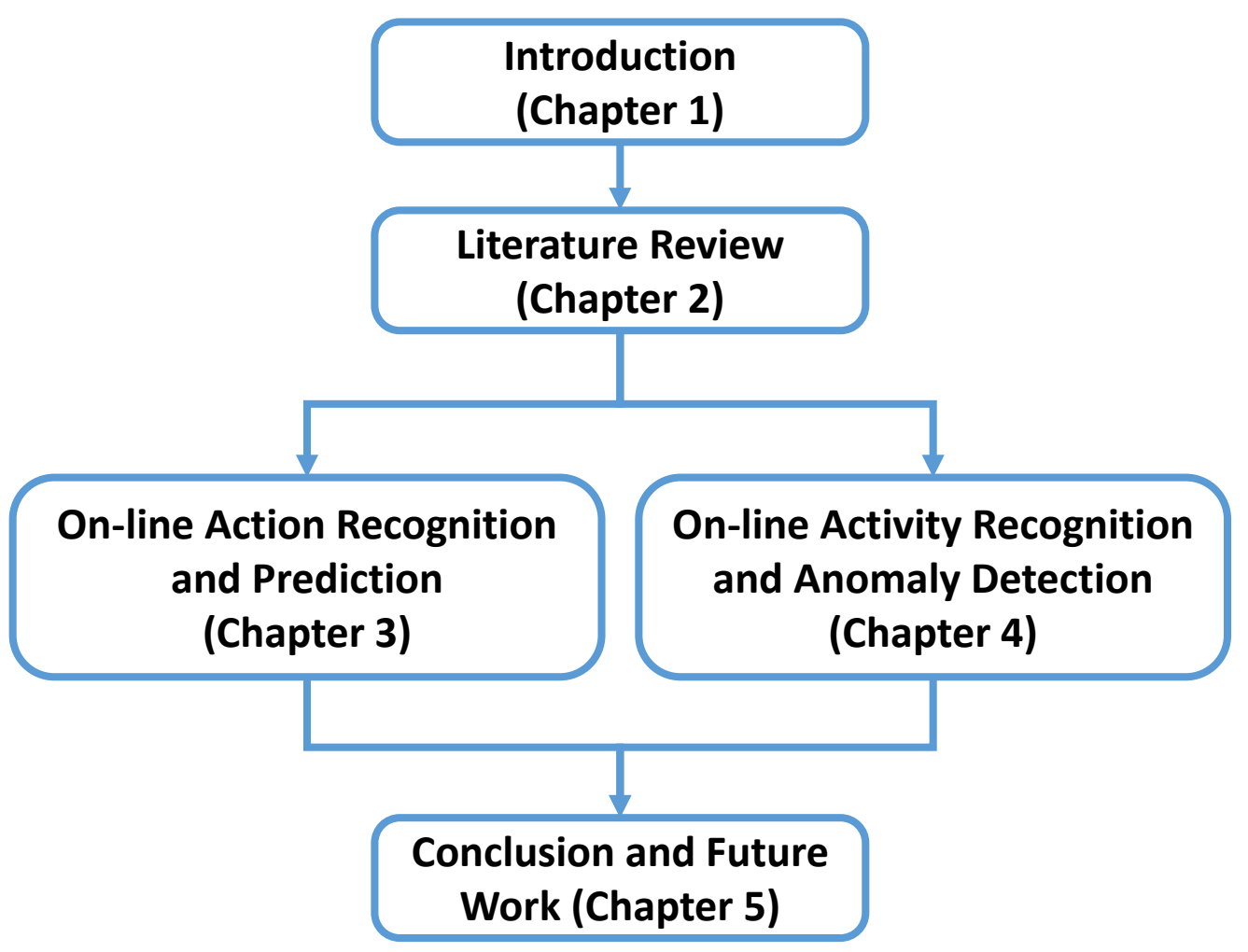

Figure 1.2: The structure of this thesis.

The proposed algorithms are evaluated on multiple tasks of on-line human action and activity analysis with different datasets, where our algorithms show excellent performance and efficiency.

\subsection{Organization of the Report}

In this chapter, we introduced the background and definition of the problems which we focus on, as well as the motivation and contribution of this thesis. Sequentially, the organization of the thesis is described as follows, which is also concluded in Figure 1.2.

Chapter 2 provides a survey on the problem of human action and activity analysis. First, some local features of videos are discussed, followed by the middlelevel representations which encode the local features in the video. Then some works 
focusing on different tasks of human action and activity analysis are introduced.

Chapter 3 firstly presents the multi-assignment BoW representation method, which improves BoW representation. Additionally, a novel approach for on-line human action recognition called Discriminative Action States Discovery is proposed, where the most discriminative action states, as well as the classifiers, are discovered simultaneously.

Chapter 4 proposes a Statistical Activity Model for joint on-line action segmentation, activity recognition, and anomaly detection. In this method, temporal dependencies of action states in every activity category are modeled and a novel alarm generation method is designed based on only positive samples.

Chapter 5 concludes this thesis while the possible future works are also discussed. 



\section{Chapter 2}

\section{Literature Review}

For any tasks aiming at analyzing human actions or activities in videos, the usual framework can be summarized as follows:

1. extract the low-level features from the training videos;

2. encode the low-level feature vectors into descriptors to represent frames or videos by middle-level representation methods;

3. train a classifier or regressor based on the training data;

4. test the videos by learned classifier or regressor.

The details of different classifiers vary a lot, which depend on the goals of different tasks. Thus, in this chapter, literature review on traditional low-level features and encoding methods will be discussed first, followed by related works on different tasks of action and activity analysis.

\subsection{Low-level Features}

Low-level features are usually extracted from small 3D patches around spatialtemporal points of a video, which are also called local features. Local features 
extraction consists of two stages: determine the location and compute the descriptor.

There are two strategies to determine the spatial-temporal locations of the local features. The first is dense sampling, which extracts local features on the points densely sampled from the video with a fixed stride length [7-9]. Wang et al. proposed Dense Trajectory Feature (DTF) [7] and Improved Trajectory Feature (ITF) [8], which both select dense sampling strategy to determine the point and generate the trajectories. The second strategy is employing interest points detector to find the locations of local features. Laptev and Lindeberg [10] proposed Spatialtemporal Interest Point (STIP) in which Harris 3D detector is employed. The extension of the detected point is estimated by maximizing the normalized Laplace operator. By employing Hessian 3D detector, Willems et al. [11] introduced a new type of STIP which is scale-invariant and densely covers the video content. Integral video which is similar as integral image is utilized in [11] to speed-up the computation. Cuboid detector is presented by Dollár et al. in [12] where the locations of interest points are detected by the response of 1D Gabor filter.

After the locations of the interest points are obtained, local descriptors are computed on 3D patches of which the centers are the prepared locations. Histogram of Gradient (HoG) was first introduced by Dalal and Triggs in [13] which showed good performance on pedestrian detection. Different from HoG, Dalal et al. [14] proposed Histogram of Optical Flow (HoF) which computes the direction of optical flow between two frames instead of the gradient of the gray image of one frame. Based on the optical flow map, Motion Boundary Histogram (MBH) $[9,14]$ can be obtained by computing the gradient on the $x$-axis (MBHx) and $y$-axis (MBHy) of the optical flow map separately. These types of local descriptor mentioned above are widely used and sometimes combined to achieve better performance since HoG is good at recording the information of appearance while $\mathrm{HoF}$ and $\mathrm{MBH}$ are good at recording the information of motion. For example, in $[7,8]$, five types of local 
descriptors (i.e.HoG, HoF, MBHx, MBHy and the shape of the trajectory) are used together to represent one trajectory.

\section{$2.2 \quad$ Middle-level Representation}

Middle-level representation is used to encode the low-level feature vectors of multiple frames or the whole video into a frame-level descriptor or a video-level descriptor. Middle-level representation is required to make different videos comparable, since the numbers of low-level feature vectors of different videos are usually different, where two videos cannot be compared.

Bag-of Words (BoW) is a widely used encoding method, which is first designed for text retrieval and introduced to computer vision by Sivic and Andrew [15]. Fisher Vector $[16,17]$ is a representation with higher dimension proposed by Perronnin and Dance, which is induced from Fisher Kernel [18] with the specific probability distribution, Gaussian Mixture Model (GMM). Similarly, Jégou et al. [19] proposed Vector of Locally Aggregated Descriptors (VLAD) which can be computed more efficiently. BoW, VLAD and Fisher Vector are all histogram based representations, which involve statistic analysis only based on the relationship among local feature vectors in the feature space. They share the same process: i. generate the codebook by clustering; ii. assign local feature vectors to one or multiple words in the codebook. The difference of BoW, VLAD and Fisher Vector is shown in the following table, where $v_{i}$ is the $i$-th bin of the histogram, $x^{(j)}$ is the $j$-th local feature vector and $\theta$ is the parameter of GMM.

Besides general middle-level representation methods mentioned above, some other methods aim at discovering the templates or discriminative patterns of $\mathrm{d}$ ifferent action categories based on the training data [20-26]. Action Bank was introduced by Sadanand and Corso [20], which is comprised of a set of individual action detectors sampled from viewpoint space and semantic space such as object 
Table 2.1: Comparison of BoW, VLAD, and Fisher Vector.

\begin{tabular}{c||c|c|c}
\hline $\begin{array}{c}\text { Middle-level } \\
\text { Representation }\end{array}$ & BoW & VLAD & Fisher Vector \\
\hline $\begin{array}{c}\text { Clustering } \\
\text { Methods }\end{array}$ & K-means & $\begin{array}{c}\text { K-means } \\
\text { or GMM }\end{array}$ \\
\hline $\begin{array}{c}\text { Assignment } \\
\text { Methods }\end{array}$ & $v_{i}=\sum_{j, N N\left(x^{(j)}\right)=c_{i}} 1$ & $v_{i}=\sum_{j} x^{(j)}-c_{i}$ & $v_{i}=\sum_{j} \nabla_{\theta} \log P\left(x^{(j)} ; \theta\right)$ \\
\hline Statistic & 0 -order & 1 -order & 1 -order, 2 -order \\
Type & & & \\
\hline
\end{tabular}

and scene. Raptis et al. [21] presented an assembly of trajectory clusters to represent the action, which is governed by a graph based on spatial and temporal constraints. A 2D histogram descriptor for the video was introduced by Narayan and Ramakrishnan in [23], where a cause and effect relationship for pairs of trajectories is measured. Ma et al. [25] explored an ensemble of discriminative structures such as space-time patches, pair-wise patches, and space-time trees to represent higher level patterns of both the action appearance and motion. Fernando et al. [24] learned the temporal evolution directions for actions and represented a video by optimized evolution direction parameters.

With the great progress made by deep learning and the development of the pretrained convolutional network models [27], researchers began to focus on learning discriminative descriptors by deep Convolution Neural Network (CNN) to represent human actions and activities. Different from convolution on images [28], videos are 3D, thus image based deep learning features are not suitable for videos due to lack of motion modeling. To overcome the gap between image convolution and video convolution, some different types of CNNs were proposed [1,29-33]. A two stream CNN was proposed by Simonyan and Zisserman in [29], where appearance and motion of actions are modeled separately and later fusion is employed to obtain 


\begin{tabular}{|c|c|c|c|c|c|c|c|c|c|c|c|c|c|c|}
\hline $\begin{array}{c}\text { Conv1a } \\
64\end{array}$ & $1=$ & $\begin{array}{c}\text { Conv2a } \\
128\end{array}$ & 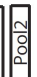 & $\begin{array}{c}\text { Conv3a } \\
256\end{array}$ & $\begin{array}{c}\text { Conv3b } \\
256\end{array}$ & & $\begin{array}{c}\text { Conv4a } \\
512\end{array}$ & $\begin{array}{c}\text { Conv4b } \\
512\end{array}$ & $\frac{1}{0}$ & $\begin{array}{c}\text { Conv5a } \\
512\end{array}$ & $\begin{array}{c}\text { Conv5b } \\
512\end{array}$ & & $\begin{array}{c}\mathrm{fc} 6 \\
4096\end{array} \mid$ & $\begin{array}{c}\text { fc7 } \\
4096\end{array}$ \\
\hline
\end{tabular}

Figure 2.1: C3D network architecture [1].

the final recognition result. A 3D Convolution Neural Network (C3D) [1] proposed by Tran et al., which uses small convolution kernels and simple network structure, achieved both good performance and small computation cost. C3D network has 8 convolution layers, 5 max-pooling layers, and 2 fully connected layers, followed by a softmax output layer, which can be observed in Figure 2.1. All 3D convolution kernels are all small kernels with the size of $3 \times 3 \times 3$ and stride 1 in both spatial and temporal dimensions. The numbers of filters are denoted in each box. All pooling kernels are $2 \times 2 \times 2$, except for pooll layer is $1 \times 2 \times 2$. The network is trained on Sports1M dataset [34]. Since the kernel size is small, it shows very good efficiency on feature extraction. As mentioned in [1], descriptors for clips with the size of $112 \times 112$ can be extracted at $313 \mathrm{fps}$ when employing K40 Tesla GPU, which is much faster than real-time.

In our research work, multiple local features and middle-level representations are employed such as BoW representation for DTF and deep learning based features such as C3D.

\subsection{Off-line Human Action Recognition}

For off-line action recognition task, training data is a set of training videos with corresponding labels of the videos. The goal is to obtain a predicted label for each of the testing videos. Given local features and video-level descriptors, a standard approach shown in Figure 2.2 is to train a classifier based on training samples and test the video-level descriptors of testing videos. SVM is the most widely used classifier for action recognition $[1,7,8,23,24]$. Linear SVM is usually employed when computation cost is a main issue while non-linear SVMs with kernel functions such 


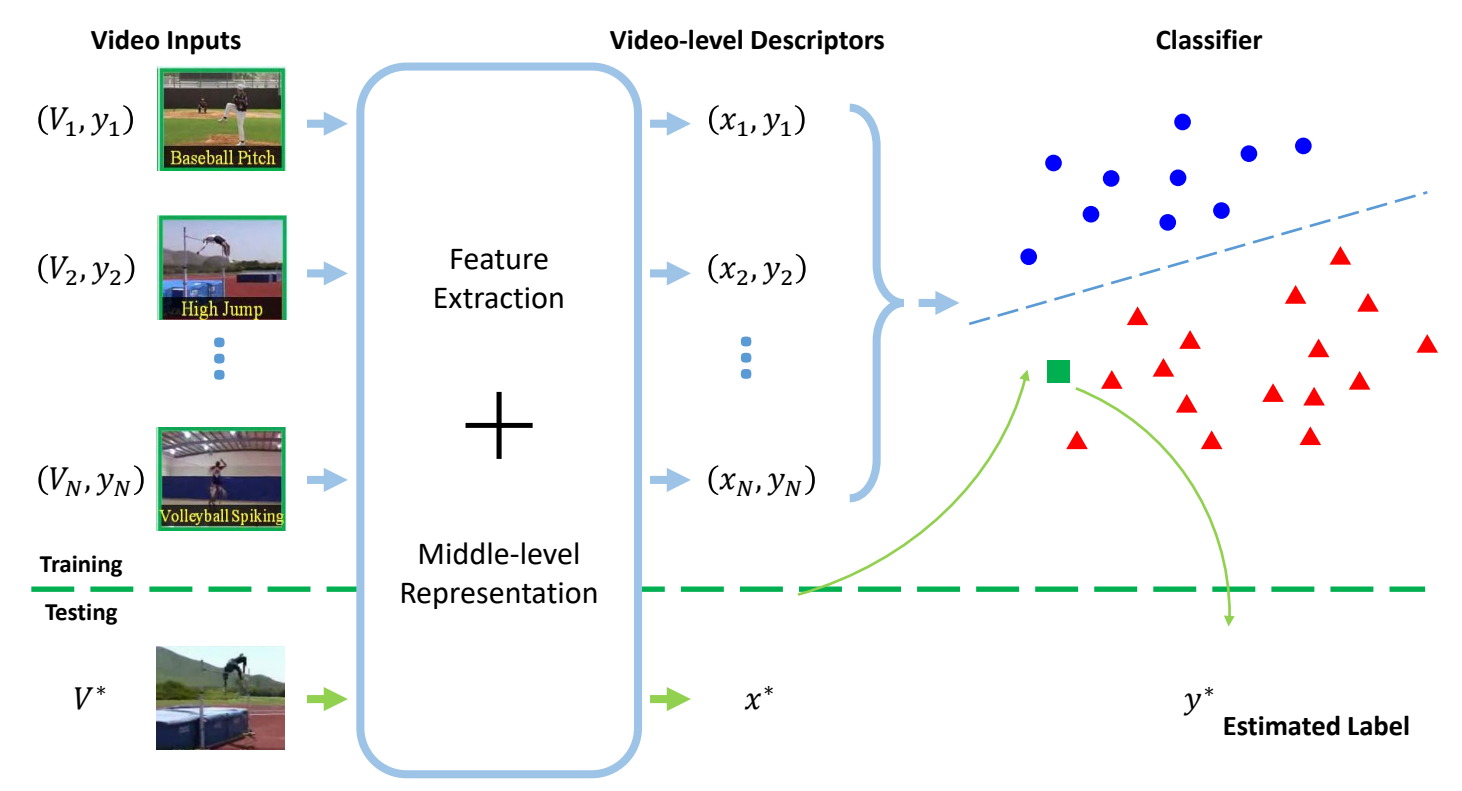

Figure 2.2: The diagram of off-line action recognition.

as widely used radial basis function (RBF) kernel are used for better performance. The details of efficiency comparison between Linear SVM and RBF kernel SVM are introduced in Section 3.5. Since we aims at building a real-time on-line action recognition system, only linear classifiers are considered.

Instead of the standard framework, other interesting works were proposed for off-line action recognition, including [35-43]. Some researchers focused on Hough transform based methods for human action recognition task [35,36]. For example, Yao et al. in [35] employed random trees to learn a mapping between local feature patches and corresponding votes. Hough transform based methods are very flexible, which not only enable action recognition but also suit for the task of action detection. Jain et al. [37] claimed that decomposing motion feature into dominant and residual motions is a better way to exploit motion. Lai et al. [38] employed multiple instances learning to find the sub-sequences in the video to recognize the events and actions. Sultani and Saleemi [39] generated the saliency map of the video and recognized actions by the detected foreground. Jain et al. [40] combined the object detection for corresponding actions since some actions involve specific objects. 


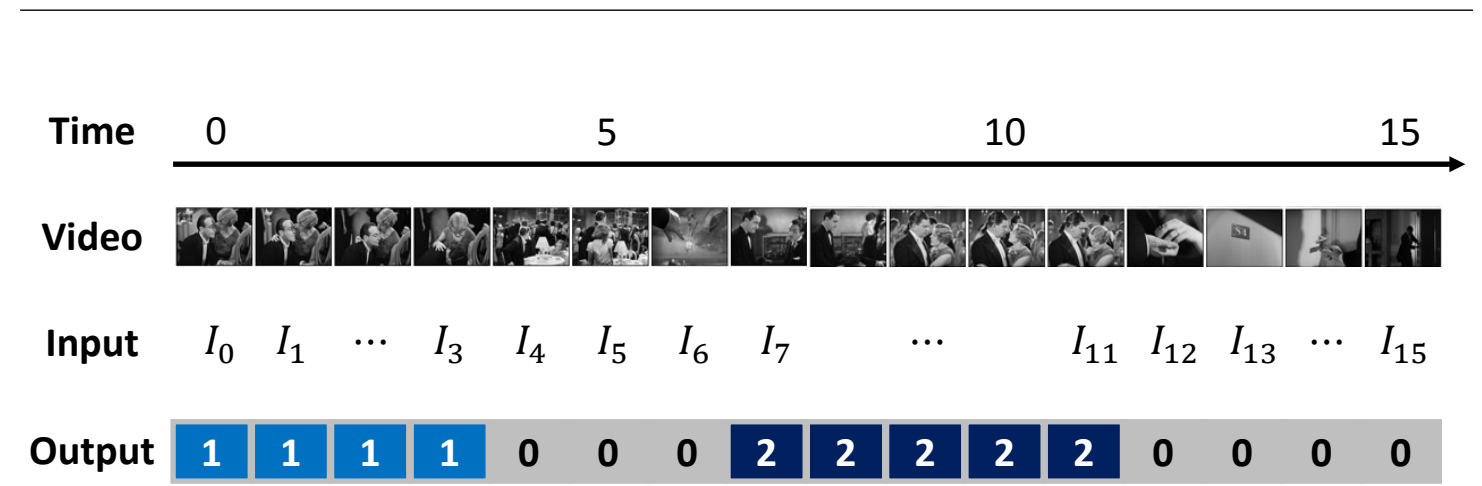

Figure 2.3: The diagram of on-line action recognition.

\subsection{On-line Human Action Recognition}

Different from off-line action recognition, the task of on-line action recognition needs to recognize what the current action is, as well as the start frame and end frame of the action in the video. Ideally, On-line means the output label of a frame should be provided when the input frame is sent to the system with no or a very small delay, as illustrated in Figure 2.3. In Figure 2.3, the output is the recognized label of the corresponded frame. Several works tried to overcome the challenges of on-line action recognition and provided good solutions for on-line action recognition [3,44-48]. Ellis et al. [44] represented an action by distinctive canonical poses based on 3D motion. Yu et al. [45] proposed an orderlet representation to capture ordinal patterns of actions. These methods $[44,45,49]$ heavily rely on depth images and skeleton features. Schindler and Van Gool introduced an approach to recognize actions by short sequences such as 1-10 frames, which is a basis for on-line action recognition. Albanese et al. [46] used a specially designed probabilistic, incremental model to describe the activity. Kviatkovsky et al. [3] computed covariance descriptors based on low-dimensional local features as frame-level descriptors and designed an incremental rule for on-going processing. However, manually annotated silhouettes or bounding boxes are required in their algorithm. 


\subsection{Human Action Prediction}

As explained in Section 1.4, action prediction is similar to off-line action recognition except that the classification is based on partial observation. This task was proposed in [50], where Ryoo introduced Integral BoW and dynamic BoW to predict the action category by matching different temporal sub-intervals. This model ignores the spatial and temporal relationship between local features so it is not discriminative enough to represent the whole action. After [50], there were some novel works focusing on action prediction such as [36,51-53]. Cao et al. [51] presented a posterior maximization approach in which action likelihood is computed by sparse coding. A maximum-margin based detector was presented by Hoai and Torre in [52] to detect early events as well as activities. Yu et al. [36] proposed a novel propagative Hough voting method to match individual local features based on random projection trees. [36] achieved very good accuracy for action prediction, especially when the observation of testing videos is nearly complete, however, when the observation is limited the performance is influenced by noise due to the lack of votes.

Table 2.2: Comparison of the task of off-line action recognition, on-line action recognition, and action prediction.

\begin{tabular}{c||c|c|c}
\hline \multirow{2}{*}{ Task } & Off-line Action & On-line Action & Action \\
& Recognition & Recognition & Prediction \\
\hline \multirow{3}{*}{ Test Videos } & Trimmed videos $x$ & Untrimmed videos $x$ & Trimmed videos $x$ \\
& with $T$ frames: & with $T$ frames: & with $T$ frames: \\
& $\left\{I_{1}, \ldots, I_{T}\right\}$ & $\left\{I_{1}, \ldots, I_{T}\right\}$ & $\left\{I_{1}, \ldots, I_{T}\right\}$ \\
\hline \multirow{2}{*}{ Inputs } & Frames: $\left\{I_{1}, \ldots, I_{T}\right\}$ & Frames: $\left\{I_{1}, \ldots, I_{t}\right\}$, & Frames: $\left\{I_{1}, \ldots, I_{t}\right\}$, \\
& Label $y$ & where $t \leqslant T$ & where $t \leqslant T$ \\
\hline Outputs & & & Label $y$ \\
\hline
\end{tabular}

Based on the above review and analysis, the difference between on-line action recognition, and action prediction is that test videos in on-line action recogni- 


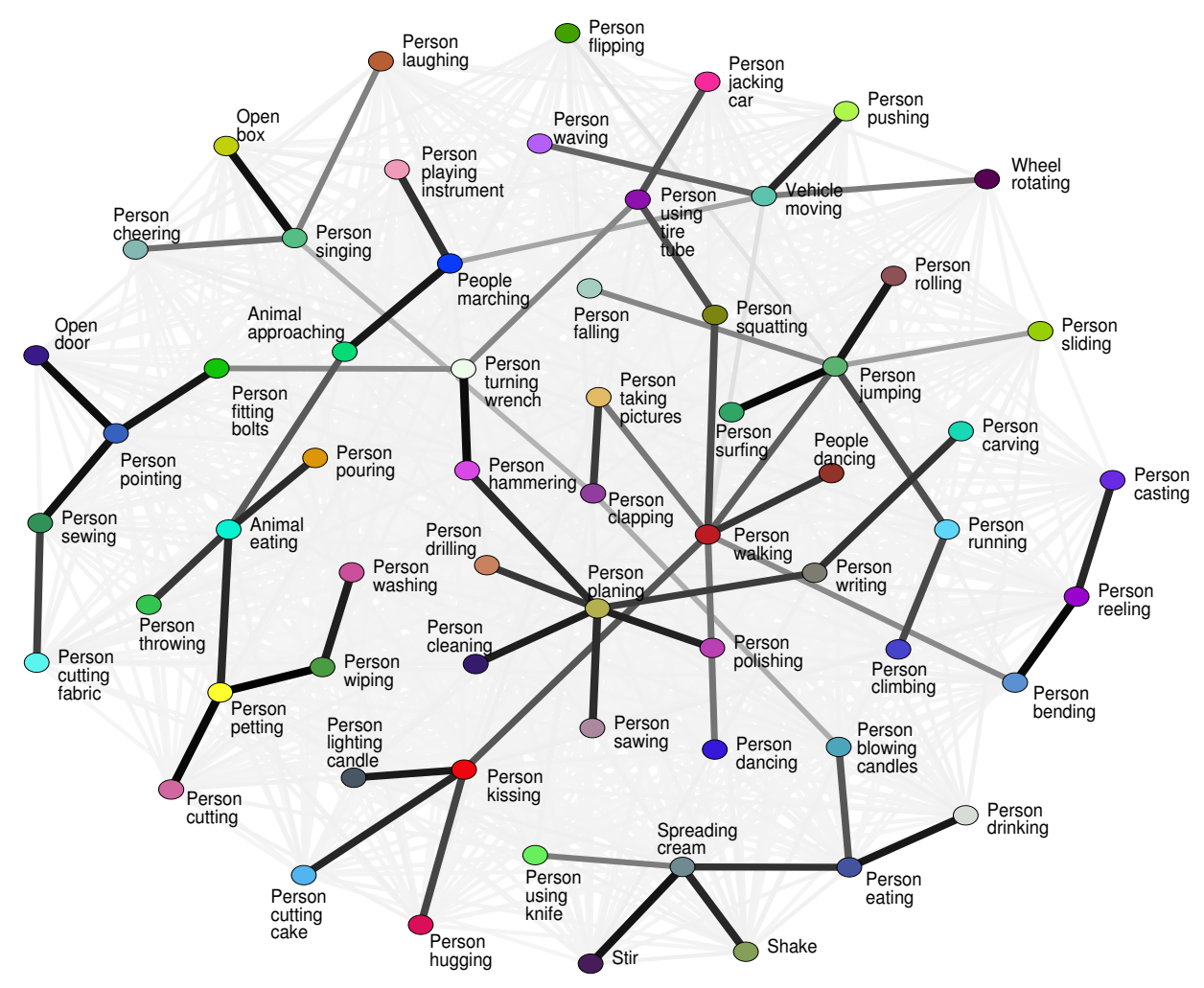

Figure 2.4: Co-occurrence graph in [2].

tion usually are untrimmed, which means algorithms for on-line action recognition should not only determine the label of each frame, but also distinguish the action from background temporally. A comparison of the task of off-line action recognition, on-line action recognition, and action prediction is shown in Table 2.2.

\subsection{Human Activity Recognition}

As mentioned in Chapter 1, human activity in this thesis is defined as a sequence of simple human action states with a flexible order. Recognizing human activities involves the information of temporal orders. Many techniques focusing on action recognition can be utilized in activity recognition since they modeled the temporal information [21,54-59]. Vail et al. [56] utilized Conditional Random Field (CRF) for temporal classification. Sum and Nevatia [57] encoded the statistical information of actions by an HMM. Raptis et al. [21] represented the video by a graphic 
model which encodes the spatial-temporal order of trajectory clusters. Niebles et al. [58] modeled activities as temporal compositions and trained discriminative models to decompose the video sequence. Gupta et al. [60] employed AND-OR graph to extract the causal relationship between action states.

For the task of recognizing long activities, several works made progress on off-line activity recognition [2,61-63]. Izadinia and Shah [2] modeled the joint relationship between action states by a co-occurrence graph, which is shown in Figure 2.4. Shi et al. [62] used Dynamic Bayesian Networks (DBN) to model the partial order of the activities and testified the algorithm on activity of blood glucose monitor calibration. Bhattacharya et al. [63] represented the activity by capturing the temporal dynamics of middle-level concepts of a window.

\subsection{Anomaly Detection and Alarm Generation}

Alarm generation is a novel task which aims to detect whether the current action state is correct according to the trained order of the current activity. Taking the activity of making milk tea as an example, the alarm for the abnormal action state should be sent by the system if previous action state is pouring the water while the current recognized state is putting in the tea bag. The order of the activity and the principle of alarm generation is shown in Figure 2.5. To the best of our knowledge,

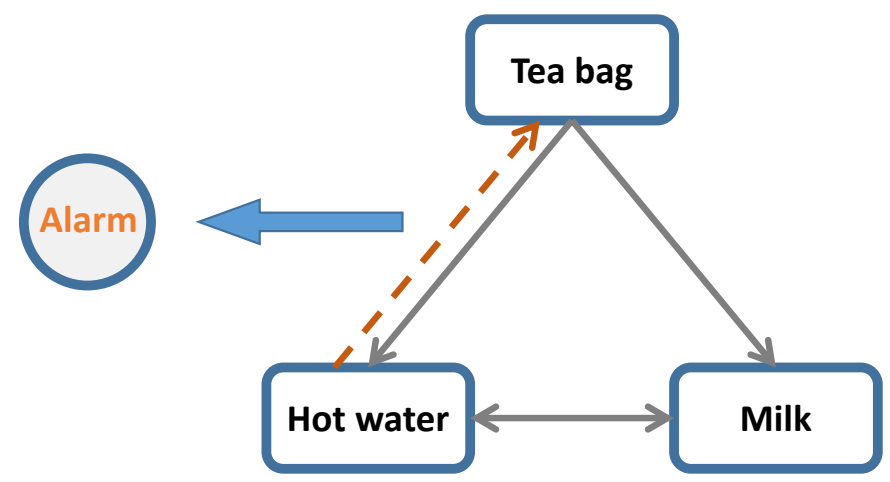

Figure 2.5: Illustration of the principle of alarm generation. 
there are few works focusing on the task of alarm generation. Soran et al. [48] designed a flexible ordered graph to represent the dependencies between action states, which determines whether to send the notification or not by computing the cost of performing one state after another. However, their approach does not allow loops, which means that all the action states can only be performed once. Besides, the method of [48] is not able to handle multiple activity categories. In this thesis, we aim to jointly recognize activities of different categories and generate alarms for the abnormal action states, which will be introduced in Chapter 4 . 



\section{Chapter 3}

\section{On-line Action Recognition}

\subsection{Introduction}

As discussed in Section 1.2, there are two main challenges when building an online human action recognition system, i.e. the noise caused by insufficient local descriptors and large intra-class variation of frame-level descriptors caused by partial observation of the video.

Every local feature vector of an action contains noise due to the variation of many factors such as action speed, spatial range, and view angle. In the task of off-line action recognition, the noise can be suppressed well by BoW encoding since BoW representation is a statistic among a temporal duration of the video and the noise follow the distribution with zero mean. For on-line action recognition, frame-level descriptors are generated from a temporal window of the video, where the local feature vectors used for encoding is much less than that in a whole video. Thus, frame-level descriptors may contain more noise than video-level descriptors. Based on this observation, to suppress the noise in frame-level descriptors, more local feature vectors are required. One intuitive way is to enlarge the length of the temporal window. However, this will increase the delay of the recognition, since for on-line recognition, each result for a temporal window can only be provided after the ending frame of the window is observed. Therefore, in this chapter, we first provide an improvement of BoW representation method to suppress the noise 


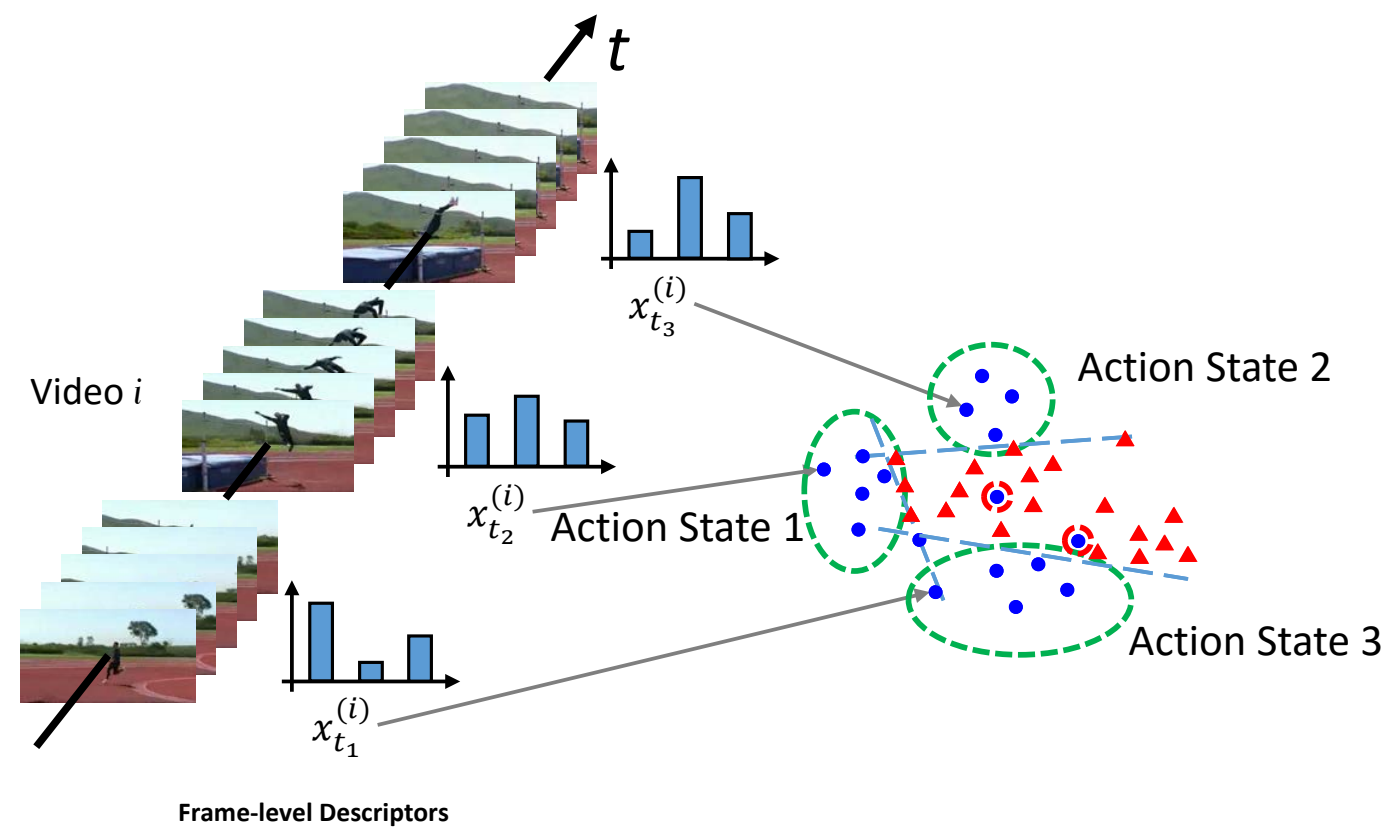

Figure 3.1: Illustration of the DASD.

of frame-level descriptors.

Furthermore, both of the motions and appearances of actions are varying temporally. Thus, there are usually multiple action states in an action, where different action states of an action obey different distributions. So in the task of on-line action recognition, frame-level descriptors of all categories of actions are distributed "chaotically", where only one linear decision boundary is not enough. Motivated by these facts, we provide a solution for on-line action recognition, where an action is treated as multiple different action states. Taking "high jump" as an example, our purpose is to find the pattern of approaching state, jumping state, and landing back state in the descriptor space. We propose a Discriminative Action States Discovery (DASD) model to assign frame-level descriptors of one category of action to different action states and learn a linear decision boundary for each action state simultaneously. The model is illustrated in Figure 3.1. In Figure 3.1, blue circles represent the positive samples while red triangles are the negative samples. The dotted circles are the regions of different action states. The dash lines are the decision boundaries for each action state. The red dash boundaries circle out the 


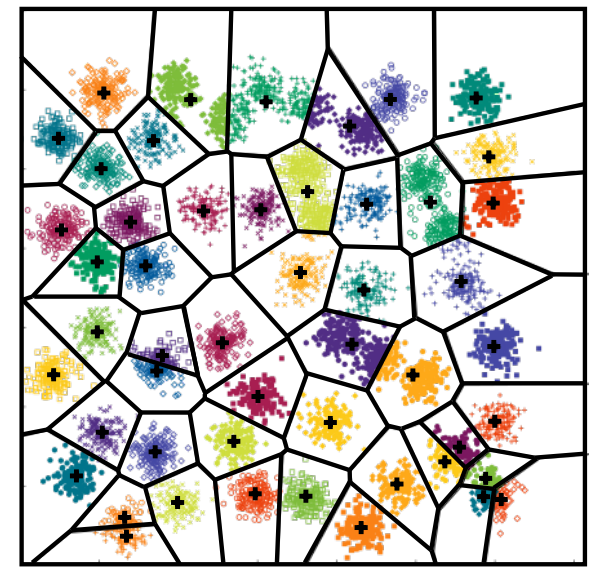

K-means

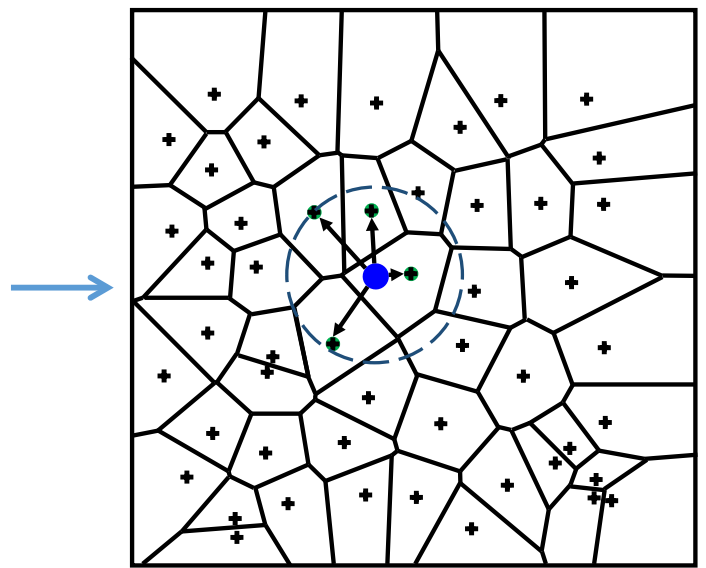

Multi-assignment (sparsity=4)

Figure 3.2: Illustration of Multi-assignment BoW representation.

ignored samples.

\subsection{Multiple Assignments for BoW Representa- tion}

\subsubsection{Model}

To address the problem of noise of frame-level descriptors, we introduce a new type of BoW representation, which assigns each local feature vector to multiple cluster centers instead of one. Given a local feature vector set $\left\{x^{(1)}, \ldots, x^{(N)}\right\}$ and the codebook $\left\{c_{1}, \ldots, c_{m}\right\}$, for classic BoW, the assignment for the $i$-th bin of the histogram is given by

$$
v_{i}=\sum_{j=1}^{N} I_{N N\left(c_{i}\right)}\left(x^{(j)}\right),
$$

where $I_{N N\left(c_{i}\right)}(x)$ is the nearest neighbor indicator function, whose value is 1 when the nearest neighbor of $x$ is $c_{i}$ among all the cluster centers in the codebook and 0 otherwise.

We improve the BoW representation by assigning each local feature vector to multiple cluster centers in the codebook based on $\mathcal{K}$-Nearest Neighbor $(\mathcal{K}$-NN) principle. The method is shown in Figure 3.2. When encoding the local feature 
vector (i.e. the blue circle in the right part of Figure 3.2), we search the $\mathcal{K}$-NN of this feature vector among the codebook and assign it to these $\mathcal{K}$-NN code words (i.e. the green dots in the right part of Figure 3.2). The number of nearest neighbors $\mathcal{K}$ determines the sparsity of the BoW vector of one local feature vector. In this figure, the sparsity is fixed by $\mathcal{K}=4$. Thus $i$-th bin of multi-assignment BoW vector is formulated as follows:

$$
v_{i}=\sum_{j=1}^{N} I_{\mathcal{K}-N N\left(c_{i}\right)}\left(x^{(j)}\right) .
$$

Similarly, $I_{k-N N\left(c_{i}\right)}(x)$ is the $\mathcal{K}$-NN indicator function.

Since local feature vectors are not sufficient in a temporal window with a fixed length, multiple assignments strategy is employed as another way to produce more similar local feature vectors. That is because assigning each local feature vector to $\mathcal{K}$ cluster centers is equivalent to generating another $(\mathcal{K}-1)$ similar vectors for each local feature vector and assigning them to the $\mathcal{K}$-NN cluster centers of the codebook.

\subsubsection{Toy Problem}

In this section, a synthetic dataset is produced to prove that multi-assignment BoW representation is capable to increase the similarity between the descriptor from one action category.

Suppose $x$ is a one-dimension local feature, where $x \in \mathbb{R}$, and a simple "action" consists of two local patterns which are modeled by Gaussian distributions $\mathscr{N}_{1}\left(\mu_{1}, \sigma\right)$ and $\mathscr{N}_{2}\left(\mu_{2}, \sigma\right)$. Based on these Gaussian distributions, 50 action examples are synthesized, where each example contains $n$ local features from $\mathscr{N}_{1}$ and $n$ local features from $\mathscr{N}_{2}$. 50 BoW vectors are obtained by BoW encoding, followed by L1-norm normalization. Let $\left\{v^{(1)}, \ldots, v^{(M)}\right\}$ denote the normalized BoW vectors, where $M=50$. The effect of noise is measured by the average of intra-class 
similarity $S_{i n}$, which is computed by:

$$
S_{i n}=\frac{1}{M(M-1) / 2} \sum_{i=1}^{M} \sum_{j=i+1}^{M}\left\langle v^{(i)}, v^{(j)}\right\rangle,
$$

where $\left\langle v^{(i)}, v^{(j)}\right\rangle$ denotes the inner product of $v^{(i)}$ and $v^{(j)}$. Intuitively, if BoW vectors contain less noise, then the average similarity $S$ will be larger.

In this toy problem, we fix $\mu_{1}=25, \mu_{2}=75$ and $\sigma=2$. The codebook with size of 100 is also fixed by $c=\{1,2, \ldots, 100\}$. Different numbers of local features $n$ and different BoW representations are tested. For classic BoW, each local feature is assigned to the nearest code word from the codebook. For multi-assignment BoW with $\mathcal{K}$, we assign each local feature to the $\mathcal{K}$ nearest codes from the codebook. It can be seen from Figure 3.3(a) that by employing multi-assignment BoW, the intra-class similarity is improved, which indicates that the effect of noise can be significantly limited especially when the local features are insufficient. When more local features are provided, the noise can also be suppressed, as discussed in Section 3.1.

The main purpose of multi-assignment is to suppress the noise for frame-level descriptors, which is indirectly helpful to classification. However, increasing the number of assignments $\mathcal{K}$ does not always mean good for the task of classification. This is because when the intra-class similarity increase, the inter-class similarity is also enlarged. If $\mathcal{K}$ is too large, on the contrary, the discrimination of BoW vectors will be weakened. In the extreme situation where $\mathcal{K}$ equals the number of code words in the codebook, each local feature vector should be assigned to all the cluster centers, where BoW vectors from different action categories become the same and the task of classification is failed. To prove this, another "action" category, which consists of local pattern $\mathscr{N}_{2}$ and $\mathscr{N}_{3}\left(\mu_{3}, \sigma\right)$, is added into this toy problem. We fix $\mu_{3}=50$. Another set of BoW vectors $\left\{u^{(1)}, \ldots, u^{(M)}\right\}$ is computed 


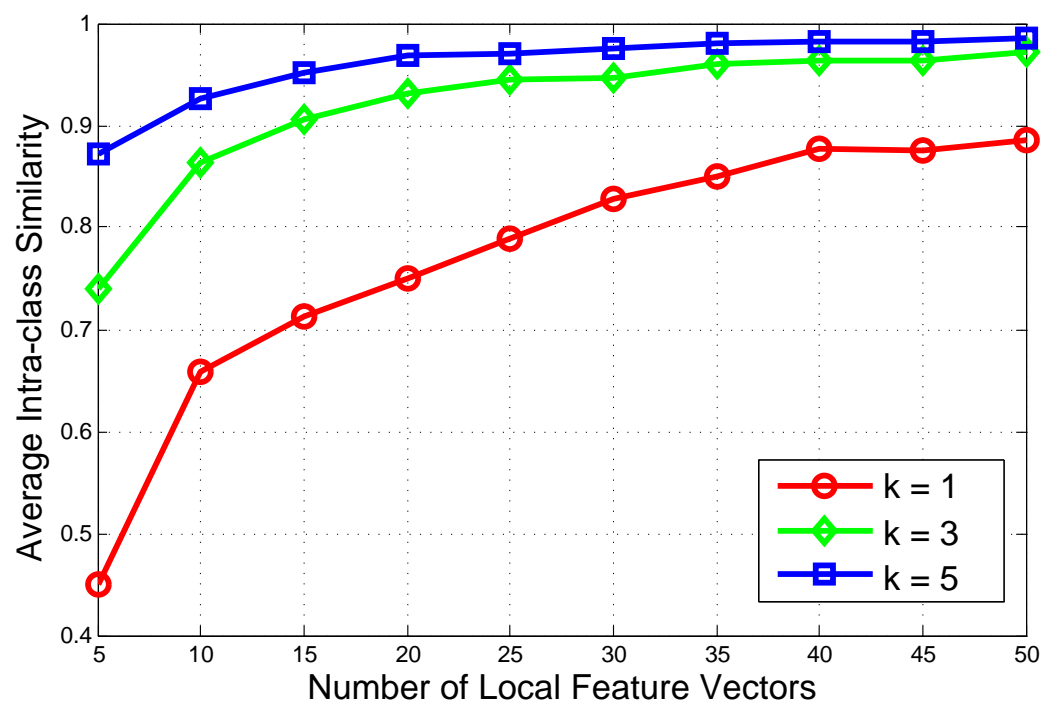

(a) Intra-class Similarity

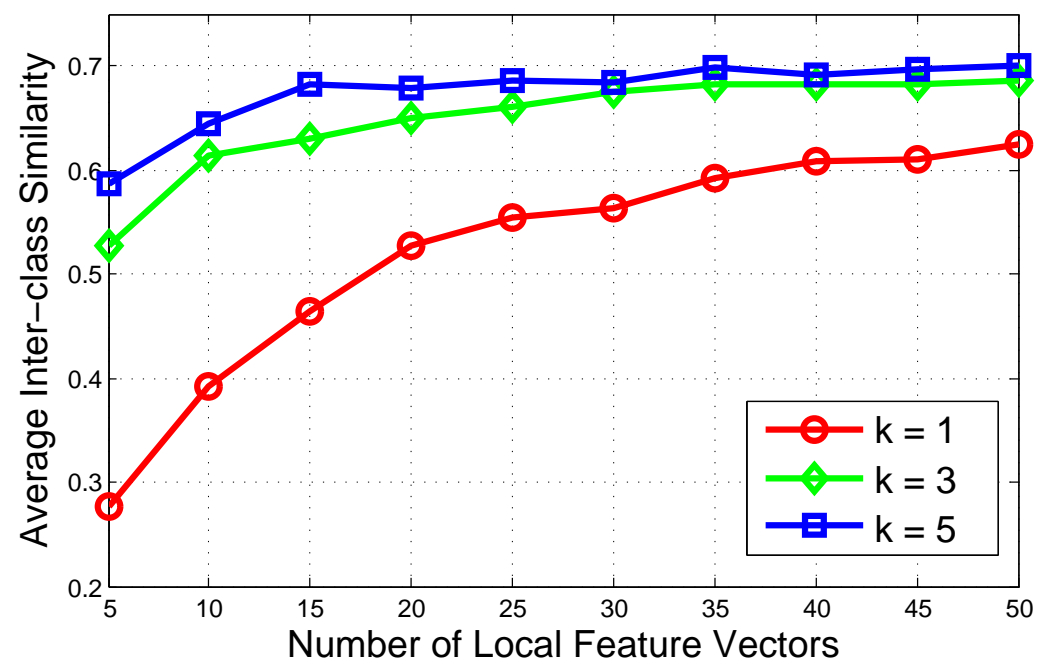

(b) Inter-class Similarity

Figure 3.3: Results on the toy problem.

in the same way. The average inter-class similarity $S_{\text {out }}$ is computed by

$$
S_{\text {out }}=\frac{1}{M^{2}} \sum_{i=1}^{M} \sum_{j=1}^{M}\left\langle v^{(i)}, u^{(j)}\right\rangle .
$$

From Figure 3.3(b) we can observe that the inter-class also increases by assigning the local feature to multiple code words. Thus, to achieve the best performance in the classification task, multiple assignments with a proper $\mathcal{K}$ is required, which is a balance for the trade-off between suppressing the noise and keeping the discrimination. 


\subsection{Discriminative Action States Discovery}

In our proposed on-line action recognition method, the temporal sliding window is employed, where each of the temporal window is represented by a frame-level descriptor. Thus the input of the training stage is a set of frame-level descriptors and decisions are made by trained classifiers based on the frame-level descriptors of the testing video. We will begin with the two-class classification where the training videos consist of positive class and negative class.

\subsubsection{Notations}

Let $\left\{V_{m}^{+}\right\}_{m=1}^{M}$ denotes the positive video set which contains $M$ videos. In each video $V_{m}^{+}$, we extract $T_{m}$ frame-level descriptors $\left\{x_{t}^{(m)}\right\}_{t=1}^{T_{m}}$ and the corresponding action state labels are $\left\{y_{t}^{(m)}\right\}_{t=1}^{T_{m}}$, where $y_{t}^{(m)} \in\{0,1, \ldots, K\}$ and $y_{t}^{(m)}=0$ means the sample $x_{t}^{(m)}$ is ignored in the training stage. $N_{+}=\sum_{m=1}^{M} T_{m}$ is the total num-

ber of positive samples. Let $\left\{x_{i}^{-}\right\}_{i=1}^{N_{-}}$denotes the negative frame-level descriptors extracted from all the negative videos and $\left\{y_{i}^{-}\right\}_{i=1}^{N_{-}}$denotes the corresponding labels, with $y_{i}^{-}=-1$. As mentioned in Section 1.2, frame-level descriptors in one class represent different action temporal patterns which result in larger intra-class variation and may cause more noise. Therefore, we propose an action states model to learn a frame-level classifier which can handle large variation and noise.

\subsubsection{Discover Single Action State}

Considering the $k$-th of the $K$ states in the positive set, the target is to maximize the margin between positive samples belonging to the $k$-th state and negative samples. A classic linear SVM with Hinge loss is employed to learn one action 
state:

$$
\begin{aligned}
& \min _{w_{k}, b_{k}} \frac{1}{2}\left\|w_{k}\right\|^{2}+\lambda \sum_{x} \epsilon_{i} \\
& \text { s.t. } \quad y_{i}\left(w_{k}^{T} x_{i}+b_{k}\right) \geqslant 1-\epsilon_{i}, \quad i=1, \cdots, N \text {, } \\
& \epsilon_{i} \geqslant 0, \quad i=1, \cdots, N,
\end{aligned}
$$

where $w_{k}$ and $b_{k}$ denote the margin and bias of SVM, $\lambda$ denotes the penalty parameter for the Hinge loss, and $\left\{x_{i}\right\}_{i=1}^{N}$ includes both of the positive samples and negative samples. We replace $\epsilon$ with the constraints and rewrite the Hinge loss with two terms $l^{+}$and $l^{-}$corresponding to positive and negative samples respectively. Then Eq. (3.5) becomes:

$$
\min _{w_{k}, b_{k}} \frac{1}{2}\left\|w_{k}\right\|^{2}+\frac{\lambda}{N_{k}} \sum_{m=1}^{M} \sum_{t=1}^{T_{m}} l_{m, t}^{+}+\frac{\lambda}{N_{-}} \sum_{i=1}^{N_{-}} l_{i}^{-},
$$

where $N_{k}=\sum_{m=1}^{M} \sum_{t=1}^{T_{m}} I\left\{y_{t}^{(m)}=k\right\}$ is the number of positive samples belonging to the $k$-th action state. $I\{\cdot\}$ is the indicator function which equals to 1 when the condition is true and 0 otherwise. the Hinge loss of positive samples $l^{+}$of $x_{t}^{(m)}$ is defined as

$$
l_{m, t}^{+}=I\left\{y_{t}^{(m)}=k\right\} \max \left\{0,1-\left(w_{k}^{T} x_{t}^{(m)}+b_{k}\right)\right\}
$$

and the Hinge loss of negative samples $l^{-}$of $x_{i}^{-}$is defined as

$$
l_{i}^{-}=\max \left\{0,1-y_{i}^{-}\left(w_{k}^{T} x_{i}^{-}+b_{k}\right)\right\}
$$

To mitigate the noise caused by the frame-level descriptors, we introduce a mechanism which can automatically detect the outliers from positive samples. The basic assumptions of this mechanism are: 1) each positive video usually contains few frame-level descriptors which are outliers; 2) videos with fewer frame-level descriptors are likely to contain fewer outliers. Based on these assumptions, we use a new term to compute the loss of outliers, which depends on the number of frame-level descriptors that a positive video contains. A proportion loss $P_{m}$ of the 


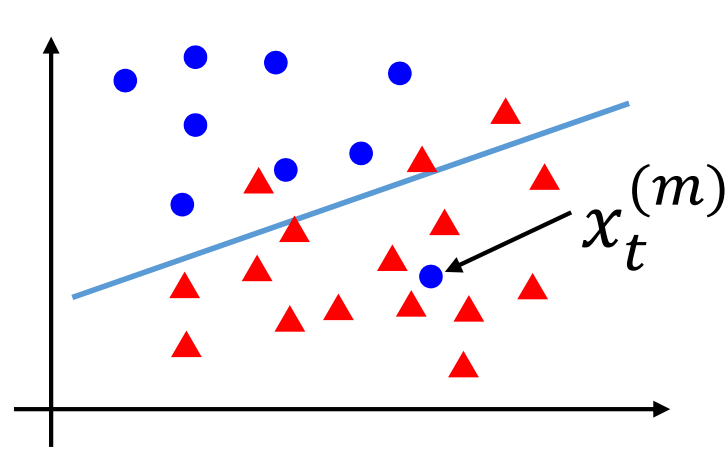

(a) considering $x_{t}^{(m)}$

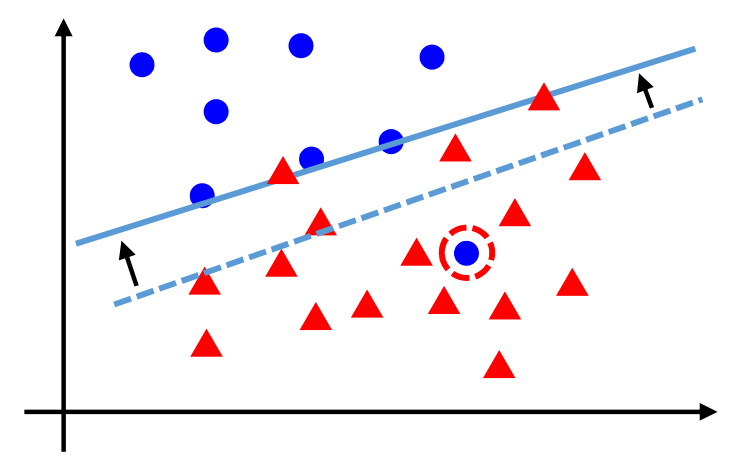

(b) ignoring $x_{t}^{(m)}$

Figure 3.4: Illustration for the ignoring mechanism.

positive video $V_{m}^{+}$is developed to limit the effect of noise samples, which is defined as

$$
P_{m}=\frac{1}{T_{m}} \sum_{t=1}^{T_{m}} I\left\{y_{t}^{(m)}=0\right\} .
$$

The summation of the proportion loss for each positive video $\sum_{m=1}^{M} P_{m}$ is used as the proportion loss term in our objective function.

Intuitively, this mechanism is to ignore the noise samples when computing the Hinge loss. By optimizing the objective function without proportion loss, the margin will be reduced and the decision boundary will be biased to noise samples (outliers) for a less Hinge loss, which is illustrated in Figure 3.4(a). Thus the ignoring mechanism is designed based on the observation as follow: the state label $y_{t}^{(m)}$ of a noise sample will be flipped from $k$ to 0 . After flipping, Eq. (3.9) is computed instead of the Hinge loss. Then the total loss on this sample is limited to $1 / T_{m}$, even this sample may violate the boundary much. And the decision boundary is adjusted to a proper position (i.e. from dash line to solid line in Figure 3.4(b)).

By incorporating the proportion loss, the objective function of single action state becomes

$$
\min _{w_{k}, b_{k}, y_{t}^{(m)}} \frac{1}{2}\left\|w_{k}\right\|^{2}+\frac{\lambda}{N_{k}} \sum_{m=1}^{M} \sum_{t=1}^{T_{m}} l_{m, t}^{+}+\frac{\lambda}{N_{-}} \sum_{i=1}^{N_{-}} l_{i}^{-}+\lambda_{p} \sum_{m=1}^{M} P_{m},
$$


where $\lambda$ and $\lambda_{p}$ control the trade-off among the regularization term ( reciprocal of margin), the margin violation term (Hinge loss), and the proportion violation term (proportion loss). In Eq. (3.10), state label of frame-level descriptor $y_{t}^{(m)}$ is also optimization variables since for each positive sample, it needs to be determined if the sample is an outlier.

\subsubsection{Discover Multiple Action States}

To discover the most discriminative states of an action, each margin between one action state and the negative action category should be maximized and the state labels of positive sample need to be determined simultaneously. To achieve this, $K$ objective functions of several individual states in Eq. (3.10) are summed with the weight $N_{k} / N_{+}$for each state $k$. In this sub-section, we will derive every term in the objective function after summing.

By summing the regularization term in Eq. (3.6) of different action states, we can obtain

$$
\begin{aligned}
\frac{1}{2} \sum_{k=1}^{K} \frac{N_{k}}{N_{+}}\left\|w_{k}\right\|^{2} & =\frac{1}{2} \sum_{k=1}^{K} \sum_{m=1}^{M} \sum_{t=1}^{T_{m}}\left\|w_{k}\right\|^{2} I\left\{y_{t}^{(m)}=k\right\} \\
& =\frac{1}{2 N_{+}} \sum_{m=1}^{M} \sum_{t=1}^{T_{m}}\left\|w_{y_{t}^{(m)}}\right\|^{2}
\end{aligned}
$$

where $w_{0}$ is a zero vector. Similarly, the Hinge loss of positive samples $L^{+}$can be rewritten as

$$
L_{m, t}^{+}=I\left\{y_{t}^{(m)} \neq 0\right\} \max \left\{0,1-\left(w_{y_{t}^{(m)}}^{T} x_{t}^{(m)}+b_{y_{t}^{(m)}}\right)\right\}
$$

To achieve the maximum margin, the Hinge loss of negative samples $L^{-}$is defined as follows:

$$
L_{i}^{-}=\max _{k}\left\{\max \left\{0,1-y_{i}^{-}\left(w_{k}^{T} x_{i}^{-}+b_{k}\right)\right\}\right\}
$$

which is the maximum of Hinge losses $l^{-}$of $K$ action states. Since the proportion loss is computed separately within each video, which is independent of the action 
state variable $k$, Eq. (3.9) does not change after summing. Based on the analysis above, Eq. (3.10) can be rewritten as

$$
\min _{w_{k}, b_{k}, y_{t}^{(m)}} \frac{1}{2 N_{+}} \sum_{m=1}^{M} \sum_{t=1}^{T_{m}}\left\|w_{y_{t}^{(m)}}\right\|^{2}+\frac{\lambda}{N_{+}} \sum_{m=1}^{M} \sum_{t=1}^{T_{m}} L_{m, t}^{+}+\frac{\lambda}{N_{-}} \sum_{i=1}^{N_{-}} L_{i}^{-}+\lambda_{p} \sum_{m=1}^{M} P_{m} .
$$

The objective function given by Eq. (3.14) is not convex. We will introduce an alternate strategy to solve the formulation later.

\subsubsection{Testing Stage}

\section{Action states classifiers}

Once the parameters of the action state model $w_{k}$ and $b_{k}$ are learned, given a test sample $x^{*}$, action state assignment is obtained by minimizing the cost of $x^{*}$ as follows:

$$
\hat{k}=\arg \min _{k} \frac{1}{2}\left\|w_{k}\right\|^{2}+\lambda \max \left\{0,1-\left(w_{k}^{T} x^{*}+b_{k}\right)\right\} .
$$

For a classic SVM, the classification result is usually determined by the sign of score $\left(w^{T} x^{*}+b\right)$. However, in our model, the objective is not the most correct action state assignment but to assign the frame-level descriptor to the action state which is most discriminative to other action categories. So when assigning the test samples, we consider both the assignment score for each action state and the margin of each action state since a larger margin means more discrimination of this action state. It can be seen from Eq. (3.15) that if a test sample satisfies that the Hinge loss equals 0 (i.e. $w_{k}^{T} x^{*}+b_{k}>1$ ) for more than one action state, this sample should be assigned to the state with the largest margin.

After assigning the testing sample to the state $\hat{k}$, the recognition score of $x^{*}$ belonging to the positive class is computed in a standard way:

$$
S\left(x^{*}\right)=\left[\exp \left(w_{\hat{k}}^{T} x^{*}+b_{\hat{k}}\right)+1\right]^{-1},
$$

where the exponential function is used to fix the score in $[0,1]$. 


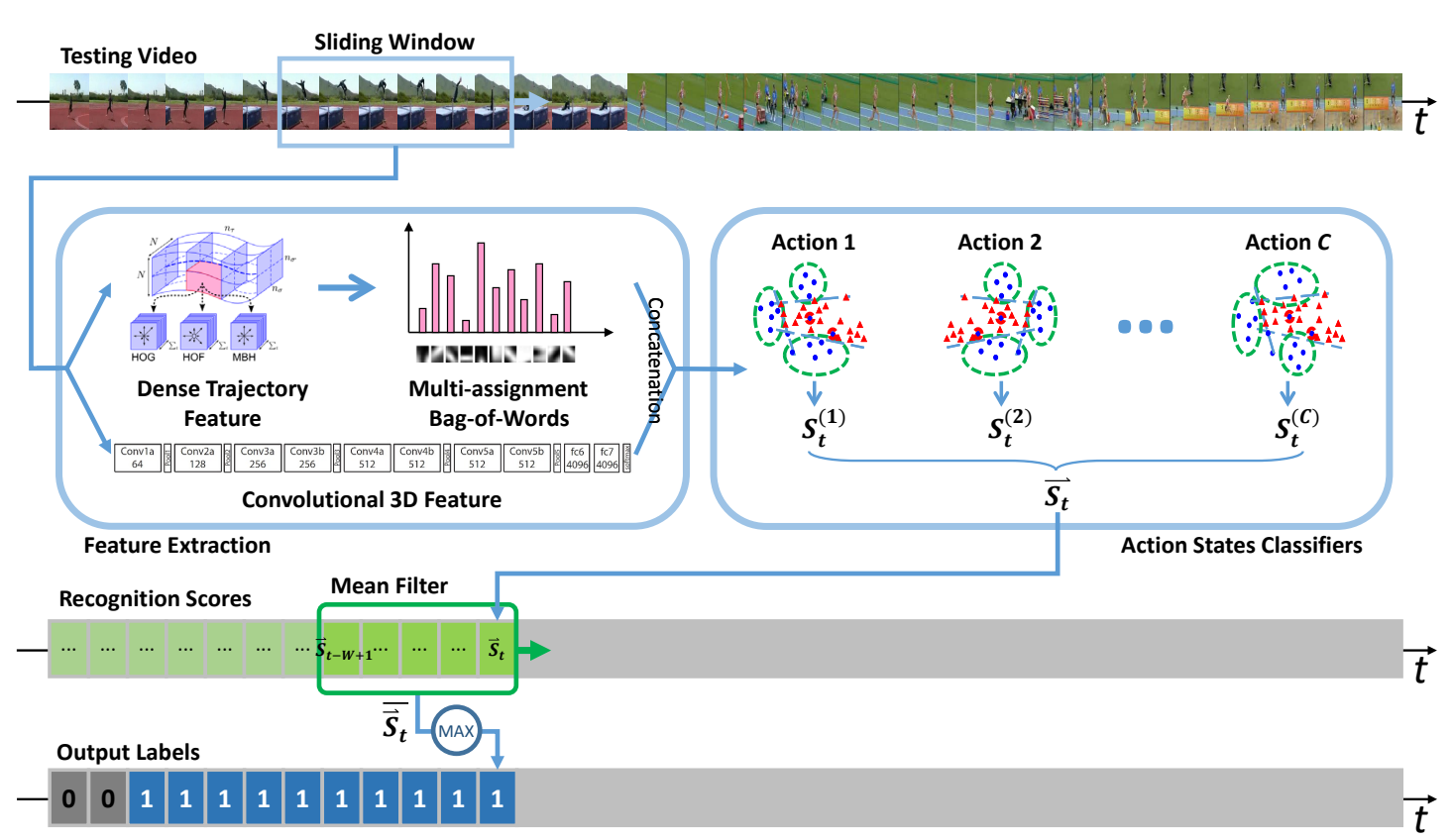

Figure 3.5: Framework of the proposed on-line human action recognition system based on DASD.

\section{On-line action recognition}

For the multi-class classification task, we utilize the one-vs-rest strategy. Suppose there are $C$ different classes of actions in the dataset. In the training stage, action states models are learned for each action class independently. In the testing stage, the recognition scores of each class are computed by Eq. (3.16) first, followed by an L1-norm normalization so that the normalized scores can be used as probabilities for the action categories that the test sample belongs to.

To avoid the sudden change of the output label, which is caused by impulse noise of the recognition score, a $W$-frame temporal smoothing window with mean filter is employed after the scores on the current frame are obtained. By doing this, the recognition scores at the current frame are smoothed by the mean of previous scores in the smoothing window. Let $S_{t}^{(c)}\left(x^{*}\right)$ denote the recognition score for the $c$-th action class at the $t$-th frame. Then the final output score for the $c$-th action 
class at the $t$-th frame is

$$
\overline{S_{t}^{(c)}}\left(x^{*}\right)=\frac{1}{W} \sum_{w=0}^{W-1} S_{t-w}^{(c)}\left(x^{*}\right),
$$

and recognition label of testing sample $x^{*}$ is given by

$$
\hat{y_{t}^{*}}=\arg \max _{c=1, \ldots, C} \overline{S_{t}^{(c)}}\left(x^{*}\right) .
$$

The framework of proposed on-line action recognition system based on Discriminative Action States Discovery, which is shown in Figure 3.5, can be summarized as follows:

1. Extract the frame-level descriptor from a temporal window ending at the current frame.

2. Classify the frame-level descriptor by DASD classifiers and obtain the recognition score for the current frame.

3. Smooth the scores in a smoothing window by a mean filter.

4. Output the smoothed scores and the label of the current frame which has the highest score.

\subsubsection{Optimization}

Since the objective function given in Eq.(3.14) is not convex, we introduce an alternative strategy to find a suboptimal solution to Discriminative Action States Discovery model. To begin the alternating procedure, the initial value of action state label $y_{t}^{(m)}$ is required. Here we follow the initialization method in [64], of which the process is listed as follows.

- train a classic linear SVM on the whole training dataset; 
- generate a residual set based on the training dataset $\left\{\bar{x}_{t}^{(m)}\right\}_{t=1}^{T_{m}}$ by

$$
\bar{x}_{t}^{(m)}:=x_{t}^{(m)}-\left(w^{T} x_{t}^{(m)}+b\right) w /\|w\|
$$

where $w$ and $b$ are the parameters of the trained linear SVM;

- split the residual set into $K$ clusters by $K$-means;

- obtain the initial state labels by assigning $\bar{x}_{t}^{(m)}$ to the cluster centers.

Algorithm 1 Discriminative Action States Discovery

Input: $\left\{x_{t}^{(m)}\right\}_{t=1}^{T_{m}}, \quad\left\{y_{t}^{(m)}\right\}_{t=1}^{T_{m}}, \quad\left\{x_{i}^{-}\right\}_{i=1}^{N_{-}}$,

iteration: $n=1$,

value of objective function: $V_{o b j}^{(0)}=\infty$

value change of objective function: $\epsilon=\infty$,

convergence threshold: $\theta=0.01$;

Output: $w_{k}, b_{k}$, where $k=1 \ldots K$.

1: while $\epsilon<\theta$ do

2: $\quad$ Update: $w_{k}^{(n)}, b_{k}^{(n)}$ by solving Eq. (3.20);

3: $\quad$ for $m=1: M$ do

4: $\quad$ for $t=1: T_{m}$ do

5: $\quad y_{t}^{(m)}=\arg \min _{k} C_{m, t}^{(k)}$;

6: $\quad$ end for

7: $\quad$ end for

8: $\quad$ Update: value of objective function $V_{o b j}^{(n)}$;

9: $\quad \epsilon \leftarrow\left(V_{o b j}^{(n-1)}-V_{o b j}^{(n)}\right) / V_{o b j}^{(n-1)}$;

10: $\quad n \leftarrow n+1$;

11: end while

12: Output: $w_{k} \leftarrow w_{k}^{(n)}, b_{k} \leftarrow b_{k}^{(n)}$.

Based on the initialization of $y_{t}^{(m)}$, the following two steps are repeated until the value of objective function converges.

1. Fix the state label $y_{t}^{(m)}$, and optimize the parameters of action states $w_{k}$ and $b_{k}$. By fixing $y_{t}^{(m)}$, the objective function becomes the summation of $K$ classic linear SVMs which is shown as follow:

$$
\min _{w_{k}, b_{k}} \frac{1}{2} \sum_{m=1}^{M} \sum_{t=1}^{T_{m}}\left\|w_{y_{t}^{(m)}}\right\|^{2}+\lambda \sum_{m=1}^{M} \sum_{t=1}^{T_{m}} L_{m, t}^{+}+\lambda \frac{N_{+}}{N_{-}} \sum_{i=1}^{N_{-}} L_{i}^{-} .
$$


Eq. (3.20) is a convex quadratic program, which can be solved by the steepest descent method.

2. Based on the current parameters of action states $w_{k}$ and $b_{k}$, optimize the state label $y_{t}^{(m)}$. Since each positive sample contributes to the Hinge lost function and the proportion loss function independently, the state labels can be optimized separately. By defining the cost of assigning sample $x_{t}^{(m)}$ to the $k$-th state as follows:

$$
C_{m, t}^{(k)}= \begin{cases}\frac{1}{2}\left\|w_{k}\right\|^{2}+\lambda \max \left\{0,1-w_{k}^{T} x_{t}^{(m)}-b_{k}\right\}, & k \neq 0, \\ \lambda_{p} N^{+} \frac{1}{T_{m}}, & k=0,\end{cases}
$$

state assignment of $x_{t}^{(m)}$ can be obtained by

$$
y_{t}^{(m)}=\arg \min _{k} C_{m, t}^{(k)}
$$

Eq. (3.22) shows that there are two types of loss for each of the positive samples (i.e the Hinge loss and the proportion loss) and the optimization procedure selects the smaller one. If $y_{t}^{(m)}=0$ then the action state label is flipped to 0 and ignored in the procedure 1 of the next iteration.

The proposed alternative optimization procedure is summarized in Algorithm 1. Experimental results show that the value of the objective function will converge in about 30 iterations.

\subsection{Experiments}

In this section, our algorithms are evaluated on multiple benchmark datasets and our privately collected dataset. We will see that our model is not only able to achieve a good performance on the task of on-line action recognition, but also suitable for the task of action prediction. 


\subsubsection{Experiment settings}

Firstly, our Multi-assignment BoW is evaluated by on-line action recognition on our privately collected dataset. The privately collected dataset consists of 160 videos containing three indoor actions: "walk around", "sit", and "squat". The three actions vary on the factors such as action speed, actors, view angles and appearance scales, which are recorded without background motion. The dataset is split into a training set and a testing set, where the testing set consists of 10 videos for each class and the training set consists of the rest 130 videos. Some examples of the privately collected dataset can be seen in Figure 3.6. The three rows of Figure 3.6 are "sit", "squat" and "walk around" respectively. DTF is utilized as local features and 1000 dimensional Multi-assignment BoW representations are encoded based on $L$-frame temporal windows, which is fixed by $L=16$ in this experiment. DTF is employed instead of ITF. Although ITF can achieve better performance, the procedure of extracting ITF includes trajectories selection and human detection, which cost much more computation resources. After middle-level encoding, a classic linear SVM is employed to classify the frame-level descriptors. 1743 BoW frame-level descriptors are extracted for each type of BoW (i.e. classic BoW and multi-assignment BoW with different $\mathcal{K}$ ), which share the same label with the video. This experiment is for the on-line action recognition, so frame-level accuracy is measured, which is the proportion of correctly classified frame-level descriptors in the testing set.

To evaluate the DASD algorithm, we also use 16-frame temporal sliding windows with an overlap of 8 frames in our model. To generate the final frame-level descriptors, we concatenate two types of frame-level descriptors:

- DTF [7] followed by 1000 dimensional Multi-assignment BoW encoding for each type of local feature (i.e. shape of the trajectory, HoG, HoF, MBHx, MBHy) and L1-norm normalization for each type, where the dimension of 


\subsection{EXPERIMENTS}
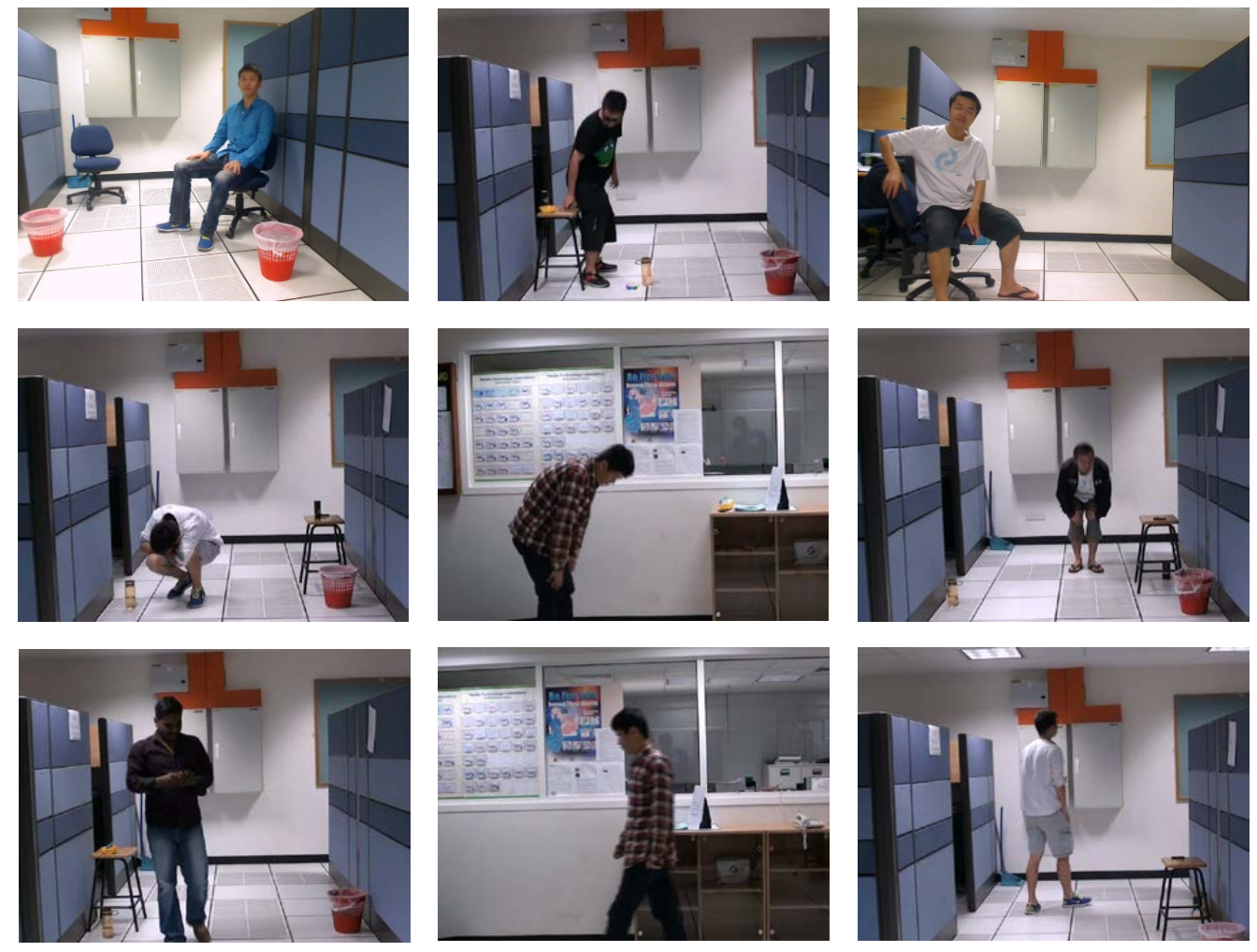

Figure 3.6: Some examples of our privately collected dataset.

frame-level descriptors is 5000 after concatenating all the five types;

- 4096-dimensional C3D feature [1], where we input video segments in temporal windows and use the outputs of the full-connect-6 (fc6) layer followed by L2norm normalization.

The parameters $\lambda$ and $\lambda_{p}$ in the objective function Eq. (3.14) are tuned based on cross validation. Classic linear SVM (i.e. $K=1$ ) is also evaluated as our baseline, which is implemented based on Liblinear [65]. We test our proposed approach on two tasks (i.e. on-line action recognition and action prediction) by three action datasets: a privately collected dataset, a subset of UCF-101 [66] and UT-Interaction [67]. 


\subsubsection{On-line Human Action Recognition}

\section{Privately collect dataset}

Multi-assignment BoW representation with different numbers of assignments $\mathcal{K}$ is evaluated and classic BoW representation is the baseline method (i.e. $\mathcal{K}=$ 1). The results are shown in Table 3.1, where it can be seen that when $k \leqslant$ 20 multi-assignment BoW outperforms classic BoW representation, and the best performance is achieved by $\mathcal{K}=4$, since multi-assignment BoW suppresses the effect of noise from frame-level descriptor. When $\mathcal{K}>20$, the accuracy drops fast as $\mathcal{K}$ increases. That is because assigning the local feature vector to too many cluster centers will eventually weaken the discrimination of frame-level descriptors as discussed in Section 3.2.2.

Table 3.1: Results of Multi-assignment BoW on privately collected dataset

\begin{tabular}{c||c|c|c|c|c}
\hline $\begin{array}{c}\text { Number of } \\
\text { Assignments } \mathcal{K}\end{array}$ & 1 & 2 & 3 & 4 & 5 \\
\hline $\begin{array}{c}\text { Average } \\
\text { Precision }\end{array}$ & 0.813 & 0.841 & 0.844 & $\mathbf{0 . 8 5 6}$ & 0.850 \\
\hline $\begin{array}{c}\text { Number of } \\
\text { Assignments } \mathcal{K}\end{array}$ & 10 & 15 & 20 & 30 & 40 \\
\hline $\begin{array}{c}\text { Average } \\
\text { Precision }\end{array}$ & 0.847 & 0.841 & 0.838 & 0.804 & 0.783 \\
\hline
\end{tabular}

Our DASD approach is also evaluated on this dataset. The results of the previous experiment show that the performance on this dataset is not so good, even when we employ the multi-assignment BoW representation. That is because all of the actions contain multiple action states and some action states are very similar in both the appearance and motion. For example, "Walk around" contains walking and turning the body, "squat" contains squatting down and standing up, "sit" contains sitting down and standing up, which is shown in Figure 3.6. From the Figure 3.6 we can see than the beginning of squatting down and sitting down 
are very similar.

We test different numbers of action states $K$ on this dataset. The experimental results shown in Figure 3.7 indicate that our approach is superior to the classic linear SVM as our model can find different action states of an action such as squatting down and standing up and model them separately. It can be observed in Figure 3.7 that for "Squat", $K=5$ is the best choice while for "Sit" the best performance is achieved when $K=2$. For all of the three action categories, the accuracy will finally decrease as $K$ increases. That is because mining too many action states will weaken the discrimination of the frame-level descriptors and the model will suffer from insufficient training samples in each action state.

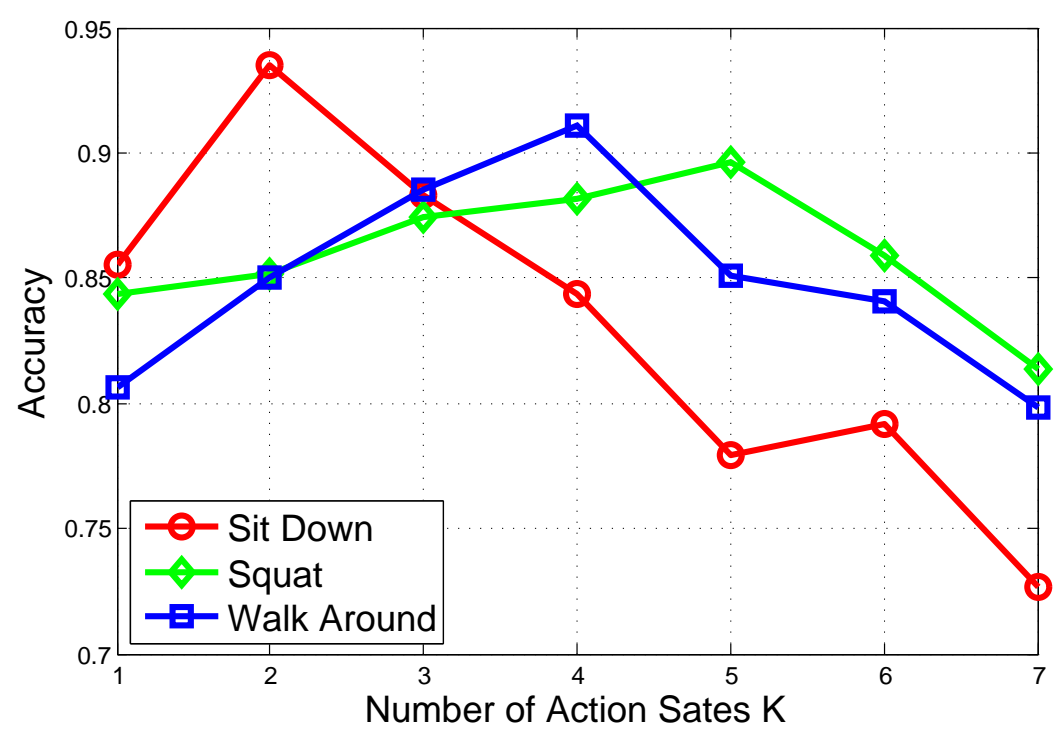

Figure 3.7: Accuracy for different numbers of action states $K$ on privately collect dataset.

Figure 3.8 shows some examples of recognition results, where three rows of results are "walk around", "squat" and "sit" respectively. From the results we can observe that almost all the actions can be recognized correctly and confidently and the output scores are stable and confident. Even some short and inconspicuous actions are also correctly classified, such as turning around between "squat". The third row, second column of Figure 3.8 shows a false result, where the beginning of "sit" is classified to "squat" when the actor is bending over. That is because "sit" and "squat" share the same state. When the actor sits on the chair, the result is 

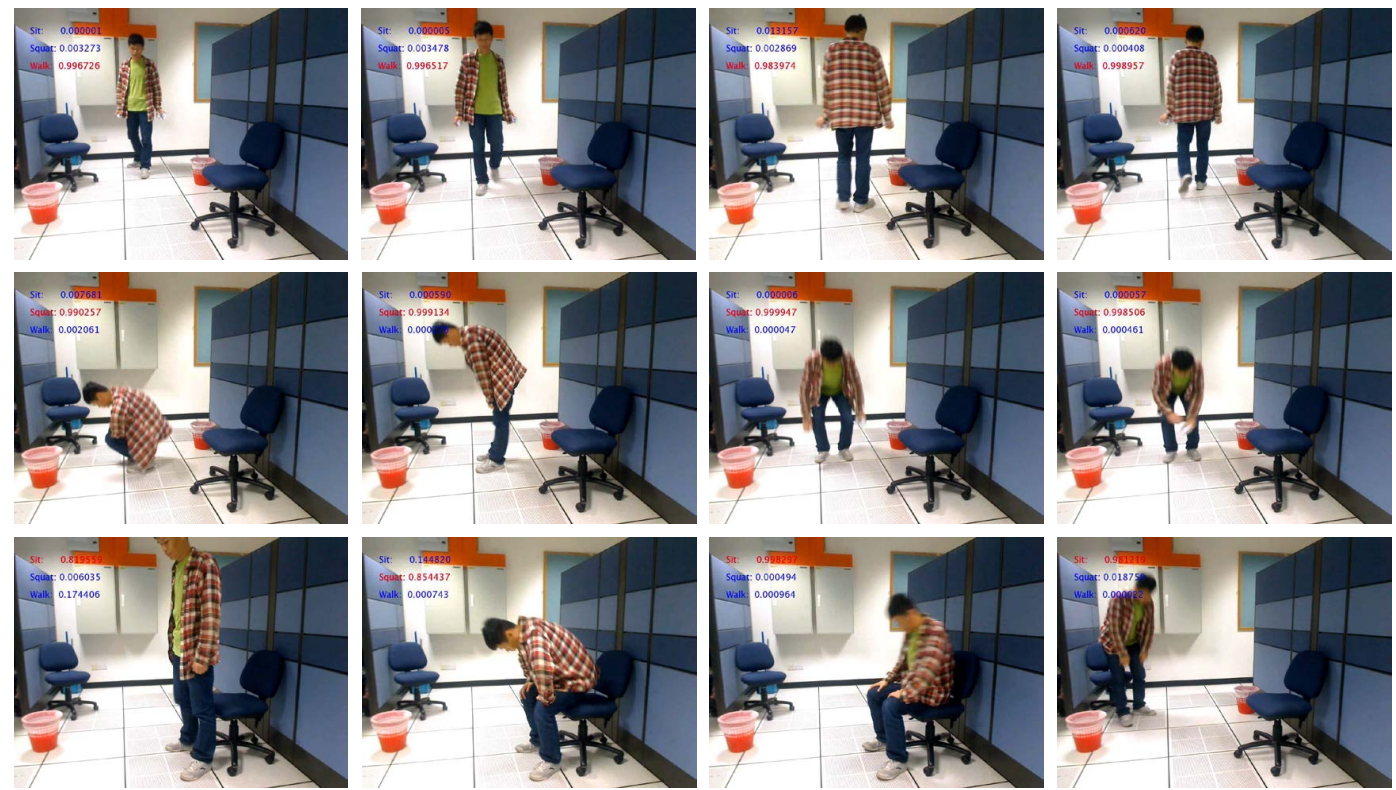

Figure 3.8: Some examples of the result on privately collect dataset.

correct again.

\section{Sub-set of UCF-101}

UCF-101 [66] is a large dataset for action recognition which is collected in the unconstrained environments and varies in viewing angle, camera motion and illumination conditions. In this experiment, we test the ability of on-line action recognition on a 20-class subset of UCF-101 [66], where the names of 20 classes are shown in Table 4.1. Since the testing videos for on-line action recognition should

Table 3.2: Names of 20-class in the subset of UCF-101.

\begin{tabular}{c|c|c|c}
\hline 7-BaseballPitch & 9-BasketballDunk & 12-Billiards & 21-CleanAndJerk \\
\hline 22-CliffDiving & 23-CricketBowling & 24-CricketShot & 26-Diving \\
\hline 31-FrisbeeCatch & 33-GolfSwing & 36-HammerThrow & 40-HighJump \\
\hline 45-JavelinThrow & 51-LongJump & 68-PoleVault & 79-Shotput \\
\hline 85-SoccerPenalty & 92-TennisSwingA & 93-ThrowDiscus & 97-VolleyballSpiking \\
\hline
\end{tabular}

be untrimmed, the testing videos of all 20 classes are randomly concatenated which 


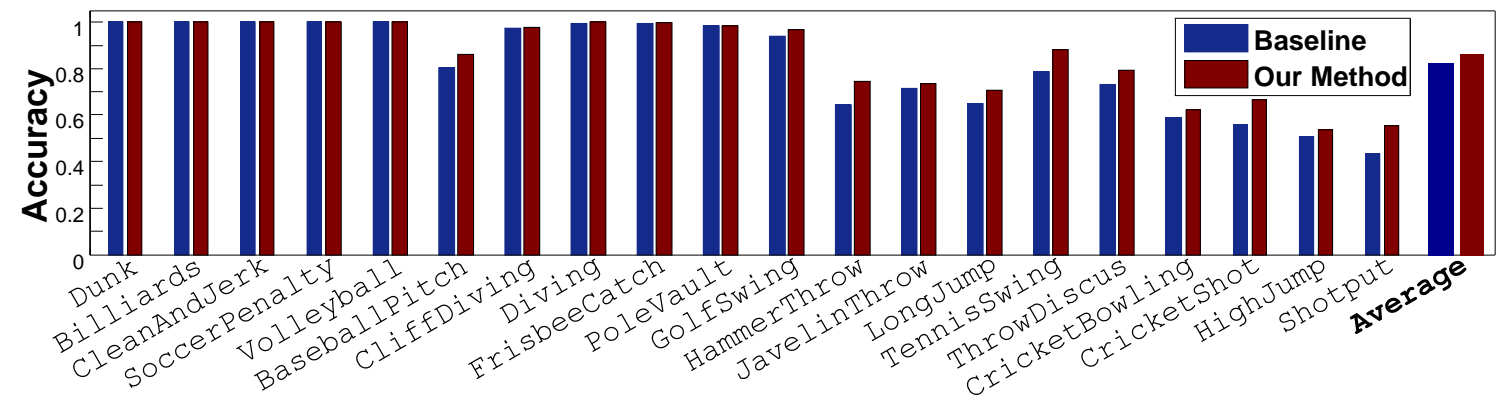

Figure 3.9: Accuracy of the 20-class of UCF-101.

means multiple actions in one testing video. The performance is evaluated by calculating the mean accuracy of every frame, where the ground truth of each frame inherits from the video label. The mean accuracy of our DASD algorithm is $85.10 \%$ while baseline method is $80.15 \%$. The results of each class and the mean accuracy of our method and baseline method are shown in Figure 3.9. The performance of different features is also evaluated. Table 3.3 shows the accuracy of our algorithm with DTF encoded by multi-assignment Bow, C3D, and the concatenation of these two descriptor. C3D is capable to capture the information from context, such as background. Therefore, C3D shows excellent performance on the subset of UCF101, in which the context and action are not independent. Furthermore, since the network of C3D is pre-trained on Sports1M dataset [34], which is very similar to the sports videos in UCF-101, the accuracy of C3D is much higher than DTF. The combination of C3D and DTF achieves the best performance, which indicates that these two types of descriptor are complementary to some degree.

Table 3.3: Experiment results on different features employed by our model.

\begin{tabular}{c|ccc}
\hline Method & DTF & C3D & Concatenation \\
\hline Accuracy $(\%)$ & 64.3 & 81.4 & 85.1 \\
\hline
\end{tabular}

To compare with [3], we follow the same setting as [3], thus eight classes "Dunk", "Cliff Diving", "Cricket Bowling", "Basketball", "Diving", "Golf Swing", "Tennis Swing" and "Volleyball Spiking" are selected. In this experiment, we test 


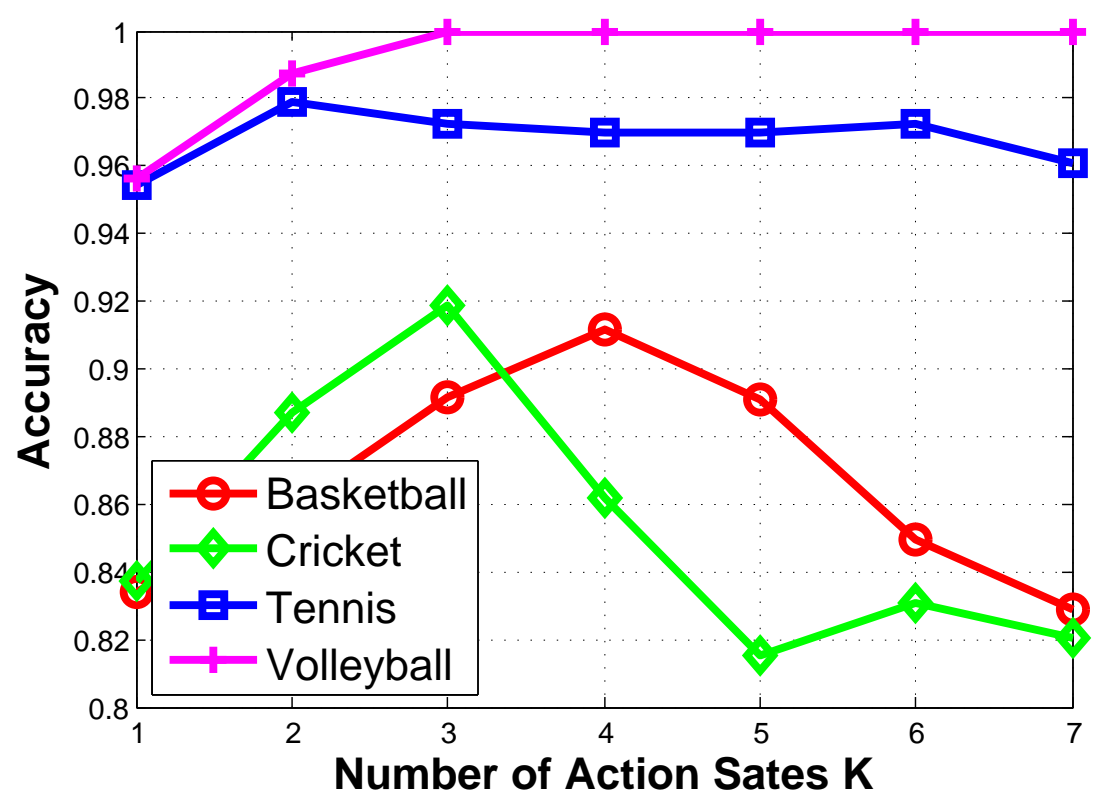

Figure 3.10: Accuracy for different numbers of action states $K$ on UCF-101.

the ability to recognize the action based on only one 16-frame temporal window of our algorithm in an on-line scenario. The performance is evaluated by calculating the rate of correctly classified frame-level descriptors. We only use C3D feature and more than 7000 descriptors are extracted for this experiment.

The performances of different numbers of action states $K$ are evaluated first, where $K=1$ means the baseline method. The results of four action classes are illustrated in Figure 3.10, while the accuracies of the other four action classes are all $100 \%$ for both the baseline and our approach. For "Basketball Shooting", $K=4$ is the best choice while for "Cricket Bowling" the best performance is achieved when $K=3$. The reason is that the intra-class variation of a simple action is not as large as the complex ones. For most of the action categories, the accuracy will finally decrease as $K$ increases, which is similar as the experiment on our privately collected dataset.

By fixing $K=3$, our algorithm is also compared with [3]. Figure 3.11 illustrates the confusion matrices of [3] and our method. The experimental results show that the accuracy of our method (DASD) achieves $97.29 \%$, which is significantly better than that of [3] (70.62\%) and the baseline (93.15\%), especially in complex action 

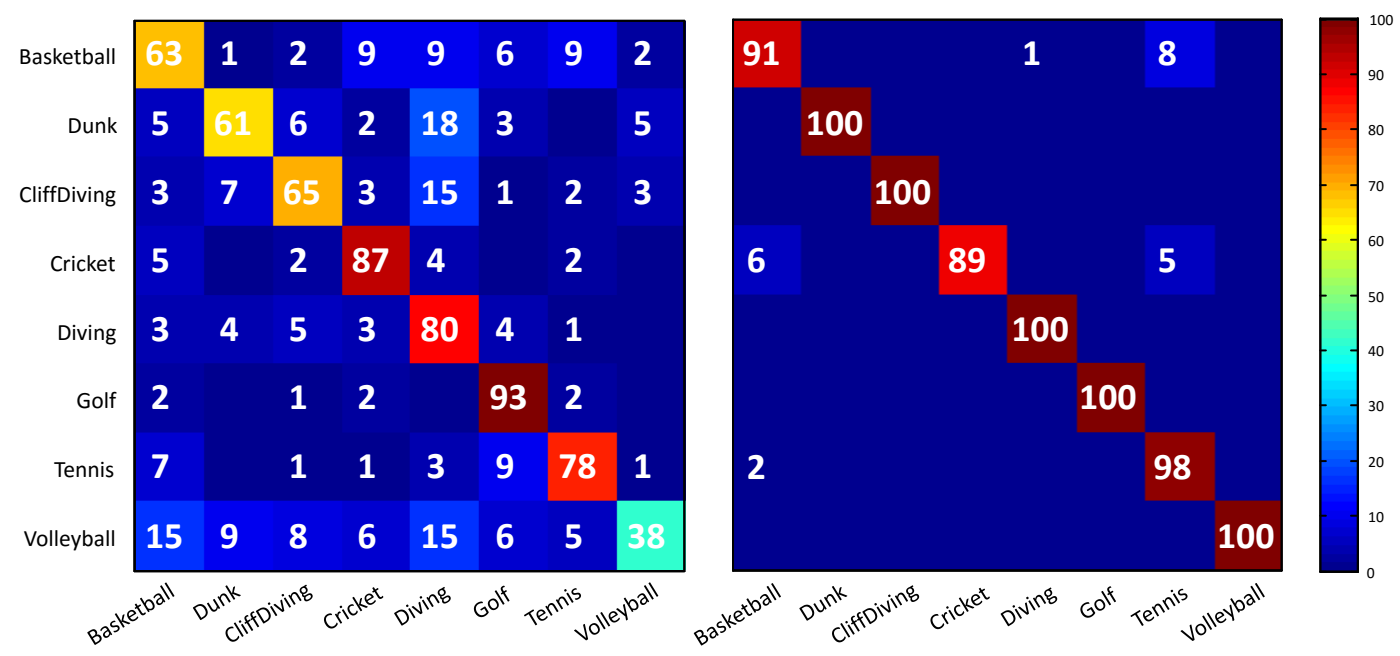

Figure 3.11: Confusion matrices of [3] (left) and Discriminative Action States Discovery (right).

categories. For example, action "Volleyball Spiking" contains passing the ball and spiking the ball. By using our approach, the accuracy of "Volleyball Spiking" achieves $100 \%$, which is about $60 \%$ higher than that of [3]. Noted that different from [3], our method provides higher classification precision without using manually annotated bounding boxes as the input.

\subsubsection{Human Action Prediction}

Our Discriminative Action States Discovery algorithm is also suitable for the task of action prediction. By employing our approach, video-level label can be obtained by a simple majority voting. Given $t$ frames from the beginning of the video, where $t \in[1, T]$, each frame label $\hat{y}_{i}^{*}$ estimated by DASD votes for the corresponding class once, and the predicted video label based on $t$ frames is given by the action class with the most votes, which is shown as follows:

$$
\hat{y}^{*}=\arg \max _{c} \sum_{i=1}^{t} I\left\{\hat{y}_{i}^{*}=c\right\} .
$$




\section{UT-Interaction}

UT-Interaction dataset [67] records six classes of actions, which consists of two subsets and each includes 10 sequences. The first subset does not contain background motion, while there is slight background motion in the second subset such as leaves. Some of the clips involve background movement and distracting people such as passing pedestrians. We follow the same leave-one-sequence-out cross validation setting as [50] on both subsets to evaluate the performance of our method on different observation ratio. The following algorithms are compared: RPT [36], Ryoo-dynamic [50], KNN-dynamic [50], SC [51] and MSSC [51].

We fixed $K=4$ in this experiment. The results are shown in Figure 3.12, where the accuracy of $10 \%$ observation ratio is not reported since for most of the videos $10 \%$ length is not sufficient to extract either DTF or C3D feature. Our approach outperforms the baseline and the state-of-the-art methods on both two sets when observation ratio is extremely limited such as $20 \%$. That is because: i) our approach is powerful to recognize any parts of the action based on discriminative action states while other approaches do not consider the intra-class variation; ii) C3D feature combines both motion and appearance of the action, which is especially good at focusing on the appearance in the first few frames and keeps the effectiveness when there are few motions [1]. When more frames of the testing video are observed, our method begins to lose the competitiveness in subset 1 and the accuracy becomes lower than [36] in subset 2 when the observation ration is larger than 0.4. Since our method ignores the relationship among the descriptors in one test video, when the test video is nearly fully observed, our frame-level prediction may not be as powerful as video-level classification like [36]. 


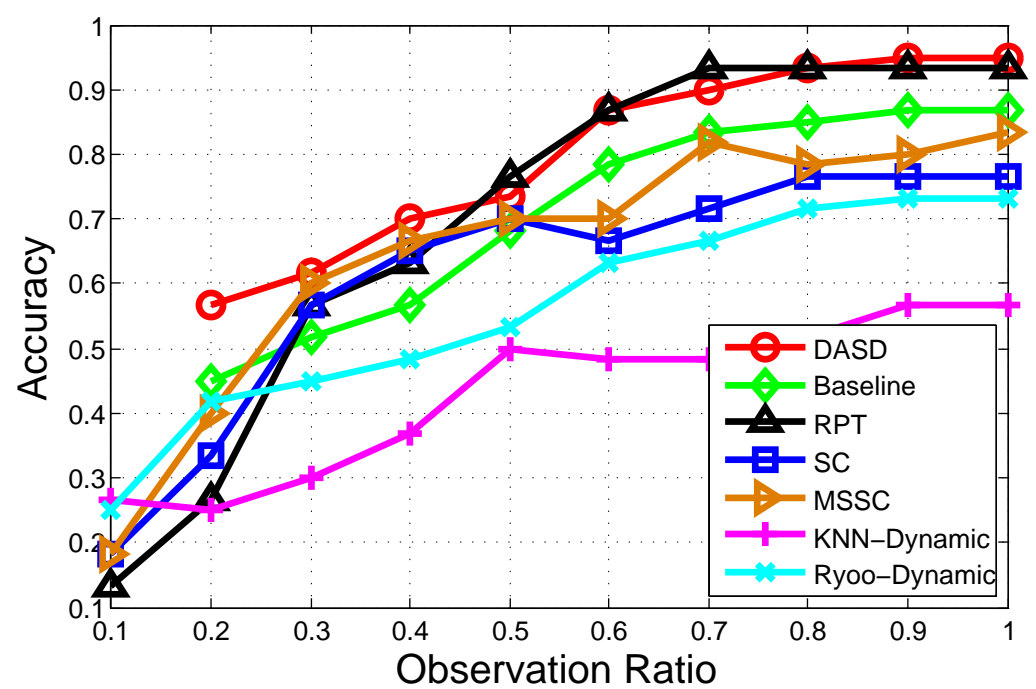

(a) Subset 1

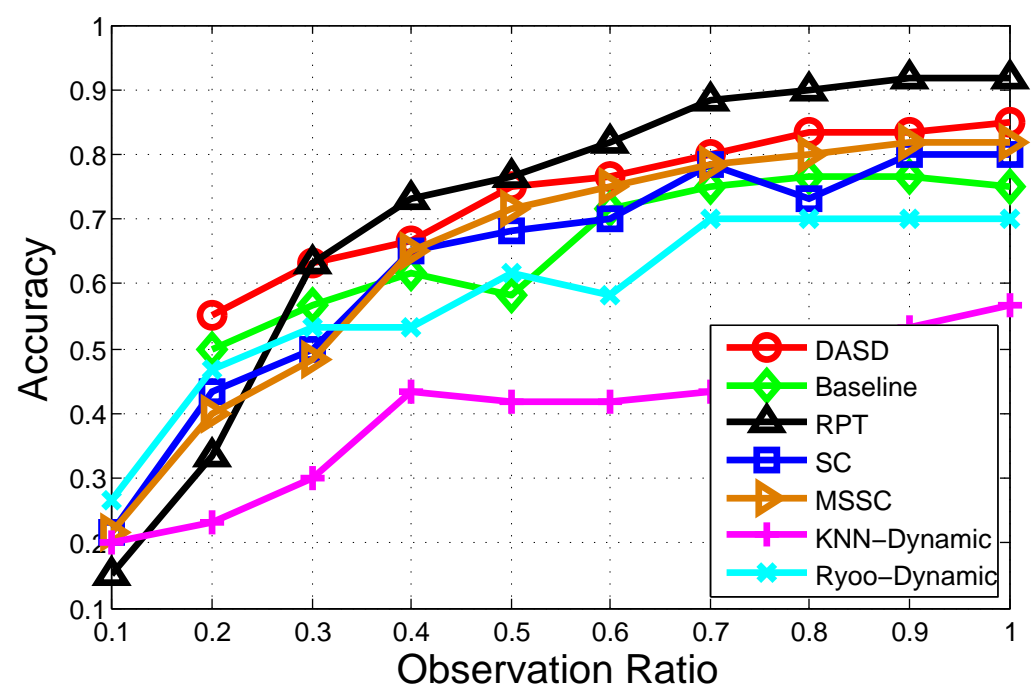

(b) Subset 2

Figure 3.12: Results of recognition accuracy based on different observation ratio on UTInteraction dataset.

\subsection{Real-time Implementation}

To achieve the real-time recognition speed, we employ DTF [7] instead of Improved Trajectory Feature (ITF) [8] since ITF includes trajectory selection which costs more time. Similarly, we utilize BoW rather than Fisher Vector as the frame-level descriptor and linear classifier instead of kernel methods. The detailed comparison of the above methods can be found in Table 3.4.

If the time complexity of $C$-class linear SVM classification is $O(C)$, for our 
Table 3.4: Comparison of time cost (ms) of different descriptors and classifiers.

\begin{tabular}{c|c|c|c}
\hline Local Features & Descriptors & Classifiers & Total Time Cost \\
\hline DTF & Multi-assignment BoW & Linear SVM & $578.61 \mathrm{~ms}$ \\
$531 \mathrm{~ms}$ & $5.68 \mathrm{~ms}$ & $0.256 \mathrm{~ms}$ & \\
\hline ITF & Fisher Vector & RBF-kernel SVM & $1725.5 \mathrm{~ms}$ \\
$1616 \mathrm{~ms}$ & $24.14 \mathrm{~ms}$ & $43.7 \mathrm{~ms}$ & \\
\hline
\end{tabular}

Table 3.5: Speed for every step in the testing stage.

\begin{tabular}{c||c|c|c|c}
\hline Step & DTF & BoW & DASD Classification & Whole Procedure \\
\hline Speed (FPS) & 29.8 & 2815.8 & $2.3 \times 10^{4}$ & 29.7 \\
\hline Step & C3D Feature & DASD Classification & Whole Procedure \\
\hline Speed (FPS) & 384 & $2.3 \times 10^{4}$ & 377 \\
\hline Step & DTF (BOW)+C3D & DASD Classification & Whole Procedure \\
\hline Speed (FPS) & 27.6 & $2.3 \times 10^{4}$ & 27.5 \\
\hline
\end{tabular}

algorithm the time complexity is $O(K C)$, where $K$ is the number of action states. In other words, the time cost of the testing stage of our algorithm approximately equals to $K C$ times 2-class linear SVM classification. Thus the recognition is very efficient. To balance the efficiency and effectiveness, every input frame is resized to $240 \times 180$ before DTF extraction. All of the algorithms are implemented by $\mathrm{C}++$ programming and tested on Core i7-4770 CPU and K80 Tesla GPU. Table 3.5 shows the detailed speed of each step of our algorithm. The average speed of DTF extraction is $30.1 \mathrm{fps}$ and the whole on-line recognition procedure is $27.5 \mathrm{fps}$, which satisfies the requirement of real-time. 


\subsection{Concluding Remarks}

In this chapter, we improve the BoW representation method and the Discriminative Action States Discovery (DASD) algorithm is proposed for on-line action recognition, which aims at discovering the most discriminative action states for each action while training the classifiers. The proposed methods are tested on the task of online action recognition and action prediction with multiple human action dataset. The results show that our approaches outperform the baseline methods on both of the tasks. The proposed algorithm also achieves the best performance among the state-of-the-arts for on-line action recognition. Based on the Multi-assignment BoW representation and DASD algorithm, an on-line action recognition system is built which achieves the real-time processing speed with a normal desktop. 



\section{Chapter 4}

\section{On-line Activity Recognition and Anomaly Detection}

\subsection{Introduction}

In this chapter, a Statistical Activity Model (SAM) will be introduced for joint activity recognition and anomaly detection in untrimmed videos. Different from human actions, as we defined in Section 1.2, an activity is more complex, which consists of a sequence of action states with a flexible order. In the task of on-line activity recognition, although the DASD algorithm proposed in Chapter 3 has the ability to discover the most discriminative action states among all categories of activities, it is still a big challenge to parse the positions of different action states. Moreover, the DASD classifiers is not capable to recognize shared action states in different activity categories. For example, there are two workout procedures which focus on upper body and lower body respectively while both of them include the state of "Plank"1. The shared action states will confuse any classifiers which classify the frame-level descriptors independently. Besides, the previous DASD algorithm does not model the temporal order information between action states so that it is impossible for this method to generate alarms for abnormal action states.

To achieve this goal, provided all the available history of an untrimmed video,

\footnotetext{
${ }^{1}$ The plank (also called a front hold, hover, or abdominal bridge) is an isometric core strength exercise that involves maintaining a position similar to a push-up for the maximum possible time.
} 


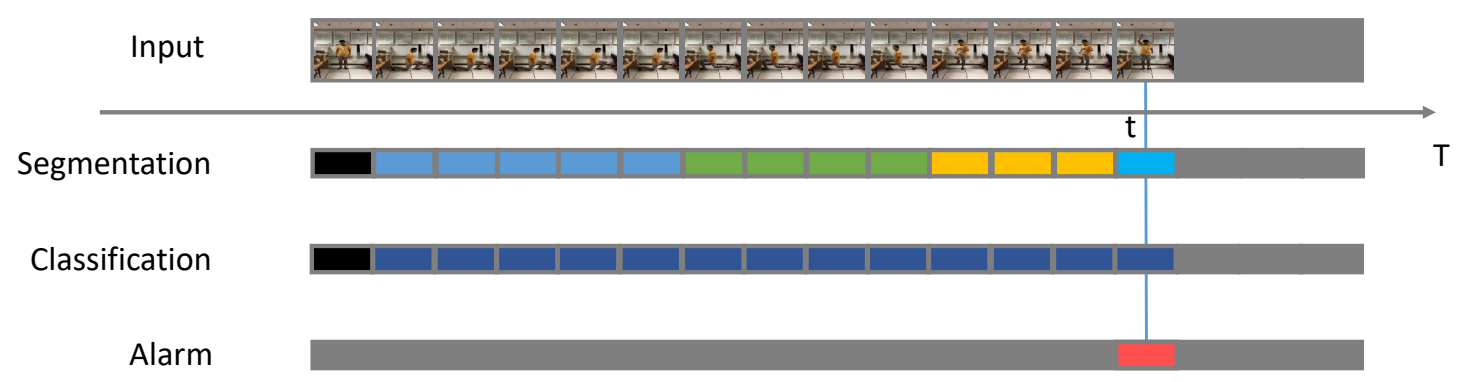

Figure 4.1: The diagram of joint action state segmentation, activity recognition, and alarm generation.

the proposed approach should tells:

1. the most probable action state label of the current frame;

2. the most probable activity label of the current frame;

3. the current action state is a normal state or an anomaly, according to the current activity category.

The framework of this problem is shown in Figure 4.1. The first output of this task needs action state recognition and temporal segmentation since we need to know not only the action state label, but also the starting and ending point of this state, which is useful for activity recognition and alarm generation. The second output requires activity recognition which is based on temporal dependencies of the action states. It is different from the task of natural language processing that activity recognition involves the length of each action state, where the transition probabilities of a specific action state with different progressions are different. To solve this problem, we proposed a novel approach which embeds the duration of each action state. The last output is determined by an alarm generation mechanism. Instead of solving these problems separately, we introduce an approach to jointly recognize activities and generate alarms for abnormal states. 


\subsection{Statistical Activity Model}

\subsubsection{Notations}

We employ temporal sliding window to extract local features and encode framelevel descriptors from the video as DASD algorithm in Section 3.3. Given a video with $T$ sliding windows, let $\left\{x_{t}\right\}_{t=1: T}$ denotes the frame-level descriptor of the $t$-th window and $\left\{y_{t}\right\}_{t=1: T}$ is the corresponding activity label of the untrimmed video, in which $y_{t} \in\{1, \cdots, C\}$. Let $\left\{S_{t}=\left(s_{t}, d_{t}, m_{t}\right)\right\}_{t=1: T}$ denotes the tuple of the current window, where $s_{t} \in\{1, \cdots, K\}$ is the action state label, $d_{t} \in \mathbb{N}^{+}$is the current duration of this action state, and $m_{t} \in \mathbb{N}^{+}$is the current state progression of the activity, which brings convenience to the alarm generation. For example, $S_{t}=(1,10,3)$ means the label of the current action state is 1 , which is the 3 -rd state of this activity and have lasted for 10 temporal windows. $\left\{A_{t}\right\}_{t=1: T}$ where $A_{t} \in\{0,1\}$ is used to represent whether the alarm signal should be sent or not at the current time.

\subsubsection{Joint Activity Recognition and Alarm Generation}

In this task, our goal is to segment the input video into action states, recognize the current activity, and determine if the current action state is an anomaly. More specifically, we aim to obtain the action state tuple $S_{1: t}$, activity label $y_{1: t}$, and the alarm signal $A_{1: t}$ based on available observation $x_{1: t}$. To achieve this, the maximum of the joint probability of $S_{1: t}, y_{1: t}, A_{1: t}$, and $x_{1: t}$ is computed, i.e.

$$
\max _{S_{t}, y_{t}, A_{t}}\left\{P\left(S_{1: t}, y_{1: t}, A_{1: t}, x_{1: t}\right)\right\}
$$

Since this problem needs to be solved in an on-line processing, we first rewrite the Eq. (4.1) with a product form over the time axis $T$ :

$$
P\left(S_{1: t}, y_{1: t}, A_{1: t}, x_{1: t}\right)=\prod_{\tau=1}^{t} P\left(S_{\tau}, y_{\tau}, A_{\tau}, x_{\tau} \mid S_{1: \tau-1}, y_{1: \tau-1}, A_{1: \tau-1}, x_{1: \tau-1}\right)
$$


in which $S_{0}, y_{0}, A_{0}$, and $x_{0}$ are introduced as virtual variables for the simplicity of the equation, which is defined as follows:

$$
P\left(S_{1}, y_{1}, A_{1}, x_{1} \mid S_{0}, y_{0}, A_{0}, x_{0}\right)=P\left(S_{1}, y_{1}, A_{1}, x_{1}\right)
$$

By assuming $x_{\tau}$ and $A_{\tau}$ are independent of the variables from the previous time, we have

$$
P\left(S_{1: t}, y_{1: t}, A_{1: t}, x_{1: t}\right)=\prod_{\tau=1}^{t} P\left(S_{\tau}, y_{\tau}, A_{\tau}, x_{\tau} \mid S_{1: \tau-1}, y_{1: \tau-1}\right)
$$

By using Bayes' Theorem three times on the temporal factors in Eq. (4.4), the equation is rewritten as

$$
\begin{aligned}
& P\left(S_{\tau}, y_{\tau}, A_{\tau}, x_{\tau} \mid S_{1: \tau-1}, y_{1: \tau-1}\right), \\
= & P\left(x_{\tau} \mid S_{1: \tau}, y_{1: \tau}, A_{\tau}\right) P\left(S_{\tau}, y_{\tau}, A_{\tau} \mid S_{1: \tau-1}, y_{1: \tau-1}\right), \\
= & P\left(x_{\tau} \mid S_{1: \tau}, y_{1: \tau}, A_{\tau}\right) P\left(A_{\tau} \mid S_{1: \tau}, y_{1: \tau}\right) P\left(S_{\tau}, y_{\tau} \mid S_{1: \tau-1}, y_{1: \tau-1}\right), \\
= & P\left(x_{\tau} \mid S_{1: \tau}, y_{1: \tau}, A_{\tau}\right) P\left(A_{\tau} \mid S_{1: \tau}, y_{1: \tau}\right) P\left(S_{\tau} \mid S_{1: \tau-1}, y_{1: \tau}\right) P\left(y_{\tau} \mid S_{1: \tau-1}, y_{1: \tau-1}\right) .
\end{aligned}
$$

Similarly, for the special case of $\tau=1$, Eq. (4.3) becomes

$$
\begin{aligned}
& P\left(S_{1}, y_{1}, A_{1}, x_{1} \mid S_{0}, y_{0}, A_{0}, x_{0}\right), \\
= & P\left(x_{1} \mid S_{1}, y_{1}, A_{1}\right) P\left(A_{1} \mid S_{1}, y_{1}\right) P\left(S_{1} \mid S_{0}, y_{1}\right) P\left(y_{1} \mid S_{0}, y_{0}\right), \\
= & P\left(x_{1} \mid S_{1}, y_{1}, A_{1}\right) P\left(A_{1} \mid S_{1}, y_{1}\right) P\left(S_{1} \mid y_{1}\right) P\left(y_{1}\right) .
\end{aligned}
$$

Based on the above analysis, Eq. (4.1) is rewritten as follows:

$$
\max _{S_{t}, y_{t}, A_{t}}\left\{\prod_{\tau=1}^{t} P\left(x_{\tau} \mid S_{1: \tau}, y_{1: \tau}, A_{\tau}\right) P\left(A_{\tau} \mid S_{1: \tau}, y_{1: \tau}\right) P\left(S_{\tau} \mid S_{1: \tau-1}, y_{1: \tau}\right) P\left(y_{\tau} \mid S_{1: \tau-1}, y_{1: \tau-1}\right)\right\} .
$$

The first component of Eq. (4.7) is the action state independent model, which computes the probability of the observation given the current action state label and the current activity label. In the next we will introduce how to convert it to a discriminative model. The second component is the alarm generation model for anomaly detection. Given the estimated labels of the activity and action states of 
all the available history, this model determines whether the alarm signal is sent or not at the current time. The third component is the action state Markov model, which is the core model to capture the temporal dependencies of action states and estimate the probability of the following action state based on the available action state sequence. The last component is Activity Smoothing Model which ensures the continuity of the activity labels within an activity.

\section{Action State Independent Model}

Since different activity categories may share one or multiple same action states, the activity label $y_{\tau}$ and action state tuple $x_{\tau}$ are weakly connected. In an extreme situation where two activities share all the action states and the only difference is temporal order, $y_{\tau}$ and $x_{\tau}$ are absolutely independent. Similarly, $A_{\tau}$ and $x_{\tau}$ are also independent. Moreover, we assume that previous action states have no impact on the observation of the current time, which is the same as HMM. Thus, in the action independent model, we have

$$
P\left(x_{\tau} \mid S_{1: \tau}, y_{1: \tau}, A_{\tau}\right)=P\left(x_{\tau} \mid S_{1: \tau}\right)=P\left(x_{\tau} \mid S_{\tau}\right)
$$

By applying Bayes' Theorem, the probability of generation of $x_{\tau}$ is rewritten as

$$
P\left(x_{\tau} \mid S_{\tau}\right)=\frac{P\left(S_{\tau} \mid x_{\tau}\right) P\left(x_{\tau}\right)}{P\left(S_{\tau}\right)}
$$

since $P\left(S_{\tau}\right)$ can be modeled by a uniform distribution in our problem and $P\left(x_{\tau}\right)$ does not affect the maximizing arguments, these two terms will be dropped in the following equations.

In our problem, we assume that the ground truth of action state labels is not available in the training stage. Thus we utilize the DASD algorithm proposed in Chapter 3 to discover the most discriminative action states for each activity category and train the action state classifiers, followed by the action state label estimation of each training sample. Then the estimated labels are treated as the 
action state ground truth of the training set. Since the observation $x_{\tau}$ has no effect on the duration of the current action state $d_{\tau}$ and the progression of the current activity $m_{\tau}$, the probability of $P\left(S_{\tau} \mid x_{\tau}\right)$ is the same as $P\left(s_{\tau} \mid x_{\tau}\right)$, which can be computed by DASD model directly in the testing stage.

\section{Action State Markov Model}

The action state Markov model is employed to compute the probabilities of assigning the current temporal window to each of the action states based on the temporal order information. In this model, we use 1-order Markov assumption, where the action state tuple $S_{t}$ is assumed to depend on its preceding one $S_{t-1}$ only. Moreover, it is assumed that $S_{\tau}$ is independent of the previous activity labels. Therefore the action state Markov model is represented as follows:

$$
P\left(S_{\tau} \mid S_{1: \tau-1}, y_{1: \tau}\right)=P\left(S_{\tau} \mid S_{\tau-1}, y_{\tau}\right)
$$

However, it is different from some classic graphical models such as HMM and CRF. Noted that $S_{t}$ is a tuple which consists of not only the label of action state but also the current duration. For example, the conditional probability given $S_{t-1}=(k, l, n)$ (i.e. $s_{t-1}=k, d_{t-1}=l, m_{t-1}=n$ ) is equivalent to the probability given $s_{t-l: t-1}=k$. In other words, we model the action state transition as well as the length of the action states.

We assume the action state Markov model is independent of the progression $m_{t}$, which is treated as a counter that

$$
m_{t}= \begin{cases}m_{t-1}, & s_{t}=s_{t-1}, \\ m_{t-1}+1, & s_{t} \neq s_{t-1},\end{cases}
$$

thus $m_{t}$ will be dropped in the following equations of this section. By replacing the tuple with action state label and the corresponding duration, the action state Markov model becomes $P\left(s_{\tau}, d_{\tau} \mid s_{\tau-1}, d_{\tau-1}, y_{\tau}\right)$ which is decomposed by Bayes's 
Theorem as

$$
P\left(s_{\tau}, d_{\tau} \mid s_{\tau-1}, d_{\tau-1}, y_{\tau}\right)=P\left(s_{\tau} \mid d_{\tau}, s_{\tau-1}, d_{\tau-1}, y_{\tau}\right) P\left(d_{\tau} \mid s_{\tau-1}, d_{\tau-1}, y_{\tau}\right)
$$

Since the duration of the action state is the current length that the action state have lasted, there are only two possible values of $d_{\tau}$ for each time step, which is constrained by

$$
d_{\tau}= \begin{cases}d_{\tau-1}+1, & s_{\tau}=s_{\tau-1}, \\ 1, & s_{\tau} \neq s_{\tau-1} .\end{cases}
$$

$d_{\tau}=d_{\tau-1}+1$ means that the previous action state continues at the current time, while $d_{\tau}=1$ means that it breaks at the current time. Thus we define a function

$$
P_{c}^{(C)}\left(S_{\tau}\right)=P_{c}^{(C)}(k, l)=P\left(d_{\tau}=l+1 \mid s_{\tau-1}=k, d_{\tau-1}=l, y_{\tau}=c\right)
$$

to represent the probability of continuation of the action state $S_{\tau}$ in the $c$-th category of activity. Similarly, The probability of break is defined as

$$
P_{c}^{(B)}\left(S_{\tau}\right)=P_{c}^{(B)}(k, l)=P\left(d_{\tau}=1 \mid s_{\tau-1}=k, d_{\tau-1}=l, y_{\tau}=c\right) .
$$

From the constraint of $d_{\tau}$ we can obtain that $P_{c}^{(C)}(k, l)+P_{c}^{(B)}(k, l)=1$.

If the action state continues, it is obvious that

$$
P\left(s_{\tau} \mid d_{\tau}=l+1, s_{\tau-1}, d_{\tau-1}=l, y_{\tau}\right)= \begin{cases}1, & s_{\tau}=s_{\tau-1}, \\ 0, & s_{\tau} \neq s_{\tau-1} .\end{cases}
$$

On the contrary, in the situation where the action state breaks at current the time step, we assume that selecting the next action state is independent of the duration. In other words, the current duration only affects the probability of continuation and break instead of which action that the next state will be. Based on this assumption, the probability of assigning the action state is

$$
P\left(s_{\tau} \mid d_{\tau}=1, s_{\tau-1}, d_{\tau-1}=l, y_{\tau}\right)= \begin{cases}0, & s_{\tau}=s_{\tau-1}, \\ \frac{T_{s_{\tau-1} \rightarrow s_{\tau}, y_{\tau}}}{\sum_{k, k \neq s_{\tau-1}} T_{s_{\tau-1} \rightarrow k, y_{\tau}}}, & s_{\tau} \neq s_{\tau-1},\end{cases}
$$


where $T_{j \rightarrow i, c}$ is the transition probability from action state $j$ to $i$ of the $c$-th category of activity. For simplicity, we define a new transition score $P_{c}^{(T)}(j, i)$, where

$$
P_{c}^{(T)}(j, i)= \begin{cases}1, & i=j, \\ \frac{T_{j \rightarrow i, c}}{\sum_{k=1, k \neq j}^{K} T_{j \rightarrow k, c}}, & i \neq j .\end{cases}
$$

Therefore, the action state Markov model is represented as

$$
P\left(S_{\tau} \mid S_{\tau-1}, y_{\tau}\right)=P_{y_{\tau}}^{(T)}\left(s_{\tau-1}, s_{\tau}\right)\left[I_{\left\{s_{\tau-1}=s_{\tau}\right\}} P_{y_{\tau}}^{(C)}\left(S_{\tau-1}\right)+I_{\left\{s_{\tau-1} \neq s_{\tau}\right\}} P_{y_{\tau}}^{(B)}\left(S_{\tau-1}\right)\right]
$$

where $I_{\{\cdot\}}$ is the indicator function which equals to 1 when the condition is true and 0 otherwise. Note that $P_{c}^{(T)}(j, i)$ actually is not a probability distribution because $\sum_{i=1}^{K} P_{c}^{(T)}(j, i) \neq 1$. In this way we can ensure that $P\left(S_{\tau} \mid S_{\tau-1}, y_{\tau}\right)$ is a probability since $\sum_{S_{\tau}} P\left(S_{\tau} \mid S_{\tau-1}, y_{\tau}\right)=1$.

\section{Activity Smoothing Model}

The activity smoothing model, which is represented by $P\left(y_{\tau} \mid S_{1: \tau-1}, y_{1: \tau-1}\right)$, aims at ensuring the smoothness of activity labels in the period of an activity. Firstly, we assume that the activity of the current time $y_{\tau}$ only depends on the variables of time $\tau-1$, hence the activity smoothing model is represented by:

$$
P\left(y_{\tau} \mid S_{1: \tau-1}, y_{1: \tau-1}\right)=P\left(y_{\tau} \mid S_{\tau-1}, y_{\tau-1}\right)
$$

Since the positions and the order of different activities in the untrimmed video are random, the probability of transition between different activities follows a uniform distribution. Within an activity, the activity labels should be the same ideally. Therefore the activity smoothing model can be represented in an intuitive way like

$$
P\left(y_{\tau} \mid S_{\tau-1}, y_{\tau-1}\right)=\left\{\begin{array}{cl}
1, & m_{\tau-1} \neq 1, m_{\tau-1} \neq M, y_{\tau}=y_{\tau-1}, \\
0, & m_{\tau-1} \neq 1, m_{\tau-1} \neq M, y_{\tau} \neq y_{\tau-1} \\
\frac{1}{C}, & m_{\tau-1}=1 \text { or } m_{\tau-1}=M
\end{array}\right.
$$


where $M$ is the total number of stages in an activity. The positions where $m_{\tau}=1$ or $m_{\tau}=M$ are treated as the boundaries between different activities. However, the model represented by Eq. (4.21) is not robust as one incorrect recognition result may mislead the following results according to the Eq. (4.21). Hence, we introduce a balance parameter $\theta \in\left[0, \frac{1}{C}\right]$ to improve the tolerability of the errors in this model, with which the activity smoothing model becomes

$$
P\left(y_{\tau} \mid S_{\tau-1}, y_{\tau-1}\right)= \begin{cases}1-(C-1) \theta, & m_{\tau-1} \neq 1, m_{\tau-1} \neq M, y_{\tau}=y_{\tau-1}, \\ \theta, & m_{\tau-1} \neq 1, m_{\tau-1} \neq M, y_{\tau} \neq y_{\tau-1} \\ \frac{1}{C}, & m_{\tau-1}=1 \text { or } m_{\tau-1}=M\end{cases}
$$

\section{Alarm Generation Model}

The alarm generation model computes the probability of sending an alarm at the current time step. We design three different types of alarm, which are described as following.

1. Abnormal action states: This alarm aims at detecting if the current action state is the correct state in progress, which checks the action state sequence without considering the duration. We represent all the transitions between different action states of an activity by a directed graph $G=(V, E)$, in which the vertices $V$ are the action states and the edges $E$ represent the transitions. If there are no loops in the graph, it is simple to tell if the action state is correct by checking if there is an edge for the current transition from $s_{\tau-1}$ to $s_{\tau}$. However, the action states in our model are automatically discovered, where it is impossible to ensure there is no loops in the transition graph. Checking current transition may miss the abnormal action state sequences. The difference between graphs with and without loops is shown in Figure 4.2. For example, there is an activity whose action state sequence is " $1 \rightarrow 2 \rightarrow 3 \rightarrow 2 \rightarrow 5$ ". An abnormal testing sample " $1 \rightarrow 2 \rightarrow 3 \rightarrow 2 \rightarrow 3$ " will be missed by checking the transitions because all the transitions appear in the 

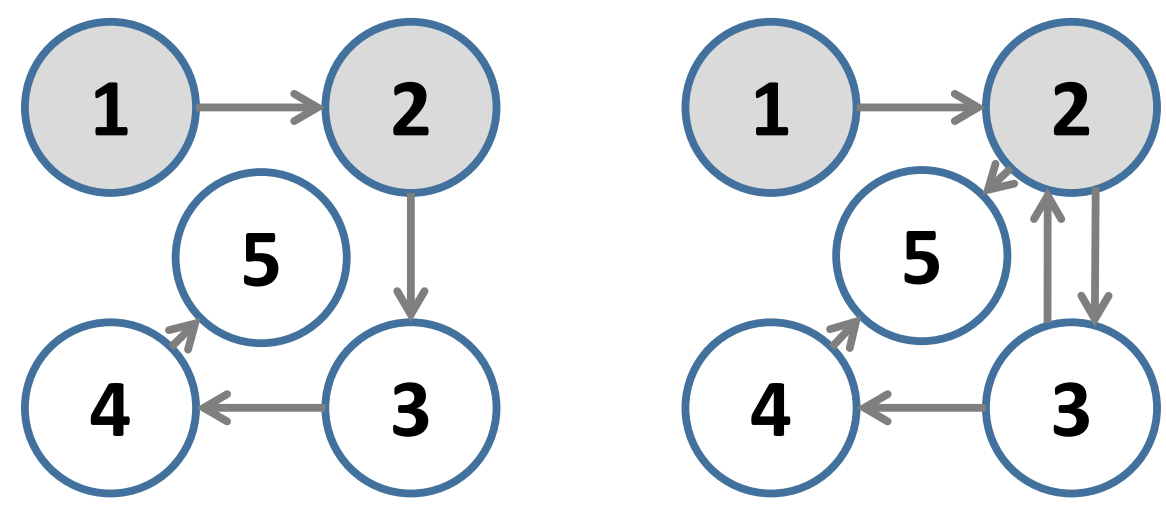

Figure 4.2: Comparison between graphs w/o loops. Left: graph without loops; Right: graph with loop $2 \rightarrow 3 \rightarrow 2$.

graph. Thus to detect abnormal action states, we need to match both the action state and its corresponding progression. To do this, a set named action state sequence pool is built for each activity. The action state sequence pool gathers all the possible action state sequences appear in the training set, which includes the action states and its action state progression. For instance, the $c$-th activity contains two different action state sequences, each of which has five different states: " $1 \rightarrow 2 \rightarrow 3 \rightarrow 2 \rightarrow 5$ " and " $1 \rightarrow 2 \rightarrow 3 \rightarrow 4 \rightarrow 5$ ". All of the training sequences begin with action state 1 , then there is only one element on progression 1 in the pool. Differently, there are two different states (state 2 and state 4 ) at progression 4. Then the pool of $c$-th activity is $\operatorname{Pool}_{c}=\{(1,1),(2,2),(3,3),(4,2),(4,4),(5,5)\}$. Thanks to the progression variable, the tuple of action state and the corresponding progression $\left(s_{\tau}, m_{\tau}\right)$ can be obtained straightforwardly from $S_{\tau}$. Therefore the probability of generating an alarm for an abnormal state is

$$
P\left(A_{\tau}^{(W)}=1 \mid S_{1: \tau}, y_{\tau}, x_{\tau}\right)=I_{\left\{\left(s_{\tau}, m_{\tau}\right) \notin \text { Pool }_{y_{\tau}}\right\}} .
$$

2. Short duration action states: The alarm for short duration action states is designed to detect if the duration of action state is too short, which works when an action state breaks. The probability of sending an alarm for overly short action 
states can be computed by the probability of Break and Continuation directly, i.e.

$$
P\left(A_{\tau}^{(S)}=1 \mid S_{1: \tau}, y_{\tau}, x_{\tau}\right)=1-P_{y_{\tau}}^{(B)}\left(S_{\tau-1}\right)=P_{y_{\tau}}^{(C)}\left(S_{\tau-1}\right) .
$$

Different from the alarm for abnormal states, the probability of generating this alarm is not binary but continuous.

3. Long duration action states: This alarm focuses on detecting the action states whose duration is too long, which is activated when the action state is continuing. Similarly, the probability of sending the alarm for overly long states can also be derived by $P_{c}^{(B)}$ and $P_{c}^{(C)}$ :

$$
P\left(A_{\tau}^{(L)}=1 \mid S_{1: \tau}, y_{\tau}, x_{\tau}\right)=1-P_{y_{\tau}}^{(C)}\left(S_{\tau}\right)=P_{y_{\tau}}^{(B)}\left(S_{\tau}\right)
$$

Note that it is an integral of the Gaussian probability distribution in the second and third types of alarm, thus we use the probability of $(\mu \pm 2 \sigma)$ as the thresholds of sending alarms. From the above analysis, we can see that in this model, $A_{\tau}$ is independent of the descriptors $x_{\tau}$ and the previous action states $S_{1: \tau-1}$, which means $P\left(A_{\tau} \mid S_{1: \tau}, y_{\tau}, x_{\tau}\right)=P\left(A_{\tau} \mid S_{\tau}, y_{\tau}\right)$. Combining all the three types of alarm leads to our alarm generation model:

$$
P\left(A_{\tau}=0 \mid S_{\tau}, y_{\tau}\right)=I_{\left\{\left(s_{\tau}, m_{\tau}\right) \in \text { Pool }_{y_{\tau}}\right\}}\left[I_{\left\{s_{\tau-1}=s_{\tau}\right\}} P_{y_{\tau}}^{(C)}\left(S_{\tau}\right)+I_{\left\{s_{\tau-1} \neq s_{\tau}\right\}} P_{y_{\tau}}^{(B)}\left(S_{\tau-1}\right)\right]
$$

Obviously, the probability of generating the alarm is:

$$
P\left(A_{\tau}=1 \mid S_{\tau}, y_{\tau}\right)=1-P\left(A_{\tau}=0 \mid S_{1: \tau}, y_{\tau}\right)
$$

\section{Final Model}

By inserting all the derivations, the final form of the proposed Statistical Activity Model is shown as follows:

$$
\max _{S_{t}, y_{t}, A_{t}}\left\{\prod_{\tau=1}^{t} P\left(A_{\tau} \mid S_{\tau}, y_{\tau}\right) P\left(S_{\tau} \mid x_{\tau}\right) P\left(S_{\tau} \mid S_{\tau-1}, y_{\tau}\right) P\left(y_{\tau} \mid s_{\tau-1}, y_{\tau-1}\right)\right\}
$$




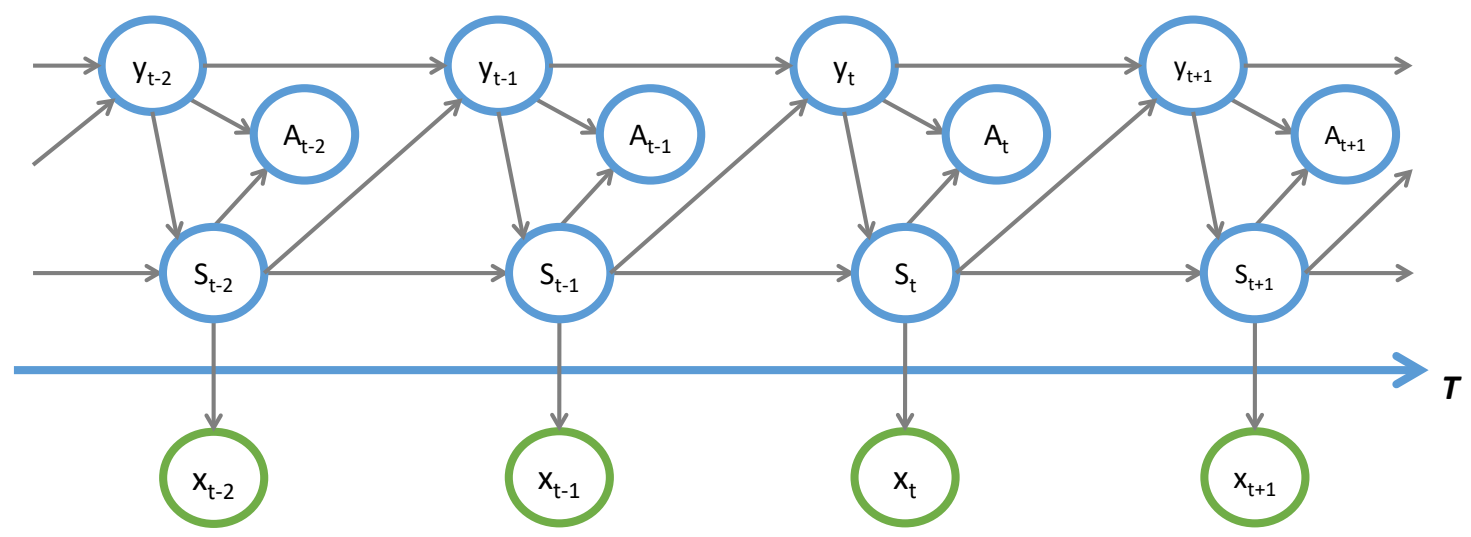

Figure 4.3: Illustration of the Statistical Activity Model from the perspective of graphic model.

where the first, third, and fourth components are represented in Eq. (4.26), Eq. (4.19), and Eq. (4.22) respectively. From the perspective of graphic model, our Statistical Activity Model can be represented by a directed graph which is shown in Figure 4.3, in which the blue arrow is the axis of time, the green circles represent the observations, blue circles are the unknown variables to be inferred, and the gray arrows mean the dependencies among the variables.

\subsubsection{Parameters Estimation}

For the action state independent model, we use the same method to train the DASD models, which is described in Section 3.3. To compute the posterior probability in (4.8), we employ a random forest trained on the training set with ground truth action state label to compute this posterior probability. The reason we use random forest instead of linear SVM is that we prefer the similar probabilities for shared action states. DASD algorithm discovers the same number of action states for every activity category. If there are shared states, for example, the first state of activity $y=1$ and the second state of activity $y=1$ ( 6 states for each activity), after gathering the action states for all activities, the state $s=1$ and state $s=8$ represent the same state. Given a descriptor of this action state, other parts of the proposed model will benefit if the output of action state independent model 
is similar, i.e. $P\left(s_{\tau}=1 \mid x_{\tau}\right) \approx P\left(s_{\tau}=8 \mid x_{\tau}\right) \approx 0.5$. Practically, random forest satisfies this requirement very well while the output scores of linear SVM usually bias towards one of the shared action states.

In the action state Markov model, the probability of continuation and breaking are determined by a probability distribution of action state length i.e. $P\left(d_{\tau} \mid s_{\tau}, y_{\tau}\right)$. Gaussian distributions $d \sim \mathcal{N}_{s, y}\left(\mu_{s, y}, \sigma_{s, y}^{2}\right)$ are employed to model the length of action states. Hence, we have

$$
P\left(d_{\tau} \mid s_{\tau}, y_{\tau}\right)=g_{s_{\tau}, y_{\tau}}\left(d_{\tau}\right)=\frac{1}{\sqrt{2 \pi} \sigma_{s, y}} \exp \left[-\frac{\left(d_{\tau}-\mu_{s \tau, y \tau}\right)^{2}}{2 \sigma_{s, y}^{2}}\right] .
$$

The parameters of Gaussian distributions are estimated by the training set of each action states and each activity category. Based on Gaussian distributions, the probabilities of breaking an action state are computed by cumulative distribution functions:

$$
P_{y_{\tau}}^{(B)}\left(S_{\tau}\right)=\sum_{d^{\prime}=1}^{d_{\tau}} g_{s_{\tau}, y_{\tau}}\left(d^{\prime}\right)
$$

and probabilities of continuation an action state are

$$
P_{y_{\tau}}^{(C)}\left(S_{\tau}\right)=1-P_{y_{\tau}}^{(B)}\left(S_{\tau}\right)=1-\sum_{d^{\prime}=1}^{d_{\tau}} g_{s_{\tau}, y_{\tau}}\left(d^{\prime}\right) .
$$

For the transition probabilities, Maximum likelihood estimation based on the action state ground truth results in a neat form of $T_{j \rightarrow i, c}$ :

$$
T_{j \rightarrow i, c}=\frac{N_{c}\{j \rightarrow i\}}{\sum_{k=1}^{K} N_{c}\{j \rightarrow k\}},
$$

where $N_{c}\{\cdot \rightarrow \cdot\}$ is the number of occurrences of the given transition in the training data from the $c$-th activity category. Therefore,

$$
P_{c}^{(T)}(j, i)=I_{\{j=i\}}+I_{\{j \neq i\}} \frac{N_{c}\{j \rightarrow i\}}{\sum_{k=1, k \neq j}^{K} N_{c}\{j \rightarrow k\}} .
$$

Practically, the probability of some specific transitions may equal to 0 since these transitions do not appear in the training set, which is detrimental to the alarm 
generation as it may eliminate the abnormal action states and results in plenty of false negative alarms. To avoid this problem, those zero probabilities are eliminated by assigning a fixed amount of probability mass $\lambda$ to the transition which does not exist in the training set. Thus the revised transition probability is

$$
P_{c}^{(T)}(j, i)= \begin{cases}1, & j=i, \\ \lambda, & j \neq i, N_{c}\{j \rightarrow i\}=0, \\ \frac{N_{c}\{j \rightarrow i\}}{\sum_{k=1, k \neq j}^{K} N_{c}\{j \rightarrow k\}}, & \text { otherwise }\end{cases}
$$

which is followed by the L1-normalization on every $i$ of $P_{c}^{(T)}(j, i), j \neq i$.

For the special case in our model where $t=1$, the probability of starting action state of the $c$-th activity is estimated by $\frac{N_{c}\left\{s_{1}=i\right\}}{\sum_{k=1}^{K} N_{c}\left\{s_{1}=k\right\}}$ to replace the term $P_{y_{\tau}}^{(T)}\left(s_{0}, s_{1}\right)$. Moreover, The conditional probability of activity label $P\left(y_{1} \mid S_{0}, y_{0}\right)$ is modeled by the uniform distribution as $P\left(y_{1} \mid S_{0}, y_{0}\right)=\frac{1}{C}$.

\subsubsection{Inference}

Our model aims to solve the action state segmentation, activity recognition and alarm generation in an on-line processing. Classic dynamic programming methods such as the Viterbi algorithm require the information of all the video, which cannot be utilized in on-line situation straightforwardly. Thus we modify the Viterbi algorithm to an on-line version. In our on-line dynamic programming algorithm, the forward recursion of each activity is computed separately, while the backward recursion, which determines $A_{\tau}, S_{\tau}, y_{\tau}$, is an activity-wise operation.

For the simplicity of equations, we define a function $\Psi_{\tau}\left(A_{\tau}, S_{\tau}, x_{\tau}, y_{\tau}, S_{\tau-1}, y_{\tau-1}\right)$ as

$\Psi_{\tau}\left(A_{\tau}, S_{\tau}, x_{\tau}, y_{\tau}, S_{\tau-1}, y_{\tau-1}\right)=P\left(A_{\tau} \mid S_{\tau}, y_{\tau}\right) P\left(S_{\tau} \mid x_{\tau}\right) P\left(S_{\tau} \mid S_{\tau-1}, y_{\tau}\right) P\left(y_{\tau} \mid s_{\tau-1}, y_{\tau-1}\right)$ 
Thus the final model in Eq. (4.28) becomes

$$
\max _{S_{t}, y_{t}, A_{t}}\left\{\prod_{\tau=1}^{t} \Psi_{\tau}\left(A_{\tau}, S_{\tau}, x_{\tau}, y_{\tau}, S_{\tau-1}, y_{\tau-1}\right)\right\}
$$

First, the forward variables are defined as

$$
\delta_{t}^{\left(y_{t}\right)}(k)=\arg \max _{S_{t-1}, y_{t-1}} \Psi_{t}\left(A_{t}, k, x_{t}, y_{t}, S_{t-1}, y_{t-1}\right) \prod_{\tau=1}^{t-1} \Psi_{\tau}\left(A_{\tau}, S_{\tau}, x_{\tau}, y_{\tau}, S_{\tau-1}, y_{\tau-1}\right) .
$$

Therefore, the recursion of forward variables $\delta_{t}^{\left(y_{t}\right)}(k)$ is derived as

$$
\delta_{t}^{\left(y_{t}\right)}(k)=\arg \max _{i, c} \Psi_{t}\left(A_{t}, k, i, x_{t}, y_{t}\right) \cdot \delta_{t-1}^{(c)}(i)
$$

All the forward variables are computed at the current time step, followed by an activity-wise L1-normalization on $\delta$, i.e. $\sum_{c=1}^{C} \sum_{k=1}^{K} \delta_{t}^{(c)}(k)=1$. Once $\delta_{t}^{(c)}(k)$ is obtained, the action state label, activity label, and alarm signal can be determined by solving the one step backward optimization:

$$
\max _{S_{t}, y_{t}, A_{t}} \delta_{t}^{\left(y_{t}\right)}\left(s_{t}\right)
$$

The proposed on-line dynamic programming method is summarized in Algorithm 2 as follows.

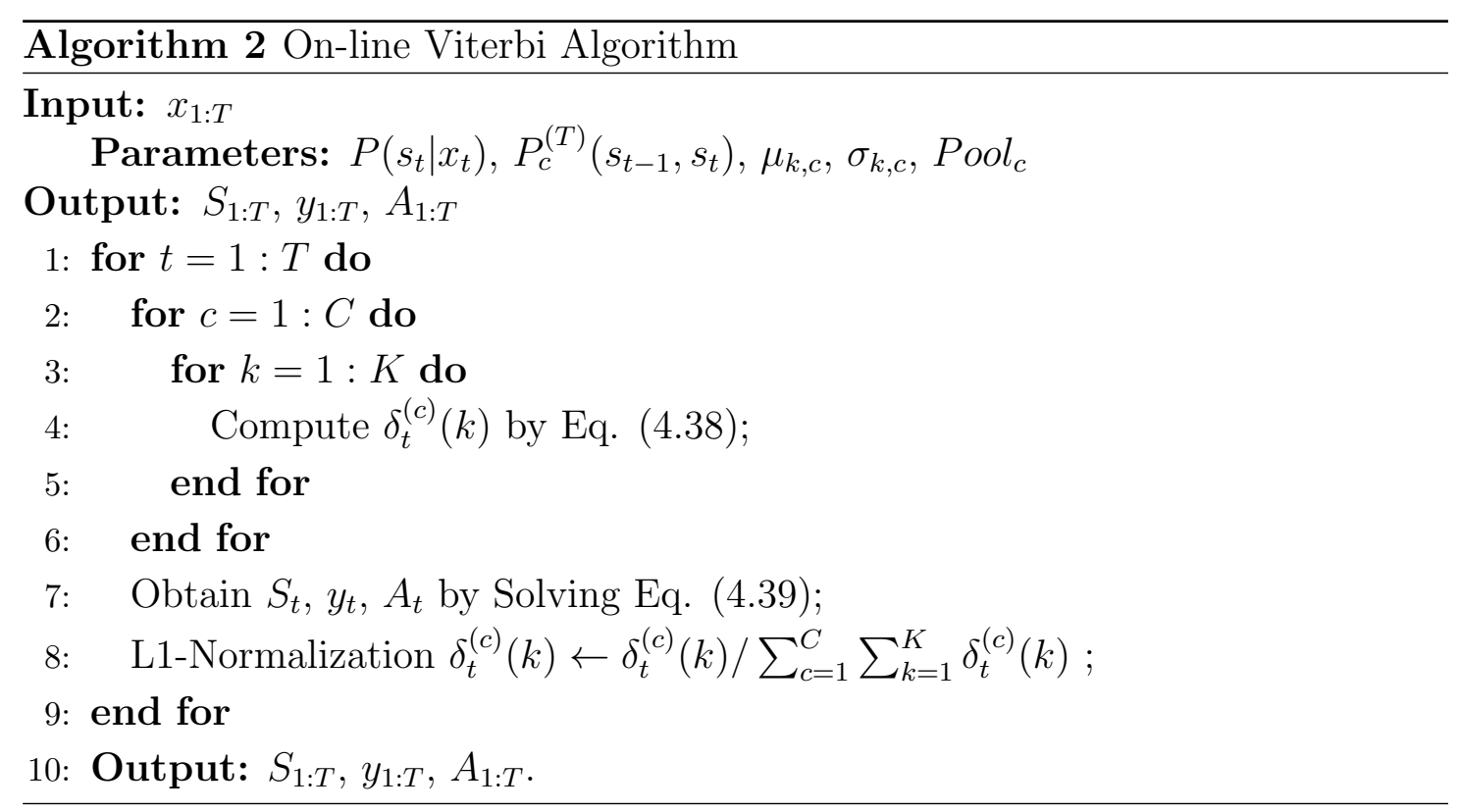




\subsection{Experiments}

We design three different experiments to evaluate the performance of action state segmentation, activity recognition, and anomaly detection respectively.

\subsubsection{Settings}

\section{Dataset}

Almost all of the existing action recognition datasets focus on short actions whose lengths are usually a few seconds. For this reason, we collect a new dataset called "WarmUp2", which includes two warm up activity categories: upper body warm up and lower body warm up. Each of the activity contains five warm up action states in the fixed order, in which some states are shared. The details of the contained action states of each activity category are listed in the Table 4.1. 14 videos of correct activities are collected, which vary in the actors, scenes, and the length of each action states. The whole length of each video is about $50 \sim 70$ seconds. Some video examples are shown in Figure 4.4, where the shared action states are marked by rectangles of the same color.

Table 4.1: Warm up activities and the contained action states in WarmUp2 dataset

\begin{tabular}{c||c|c|c|c|c}
\hline Action State Order & 1 & 2 & 3 & 4 & 5 \\
\hline Lower body & $\begin{array}{c}\text { Left Split } \\
\text { warm up }\end{array}$ & $\begin{array}{c}\text { Right Split } \\
\text { Squat }\end{array}$ & $\begin{array}{c}\text { High } \\
\text { Knees }\end{array}$ & Squat & $\begin{array}{c}\text { Shoulder } \\
\text { Stretch }\end{array}$ \\
\hline Upper body & $\begin{array}{c}\text { Right Back } \\
\text { warm up }\end{array}$ & $\begin{array}{c}\text { Left Back } \\
\text { Stretch }\end{array}$ & $\begin{array}{c}\text { Shoulder } \\
\text { Stretch }\end{array}$ & $\begin{array}{c}\text { Chest } \\
\text { Stretch }\end{array}$ & $\begin{array}{c}\text { High } \\
\text { Knees }\end{array}$ \\
\hline
\end{tabular}

\section{Feature and Parameters}

DTF is employed as local features and Multi-assignment BoW encoding are performed to generate frame-level descriptors, which are encoded based on 32-frame temporal windows with 16-frame overlap. The number of BoW assignments is fixed 


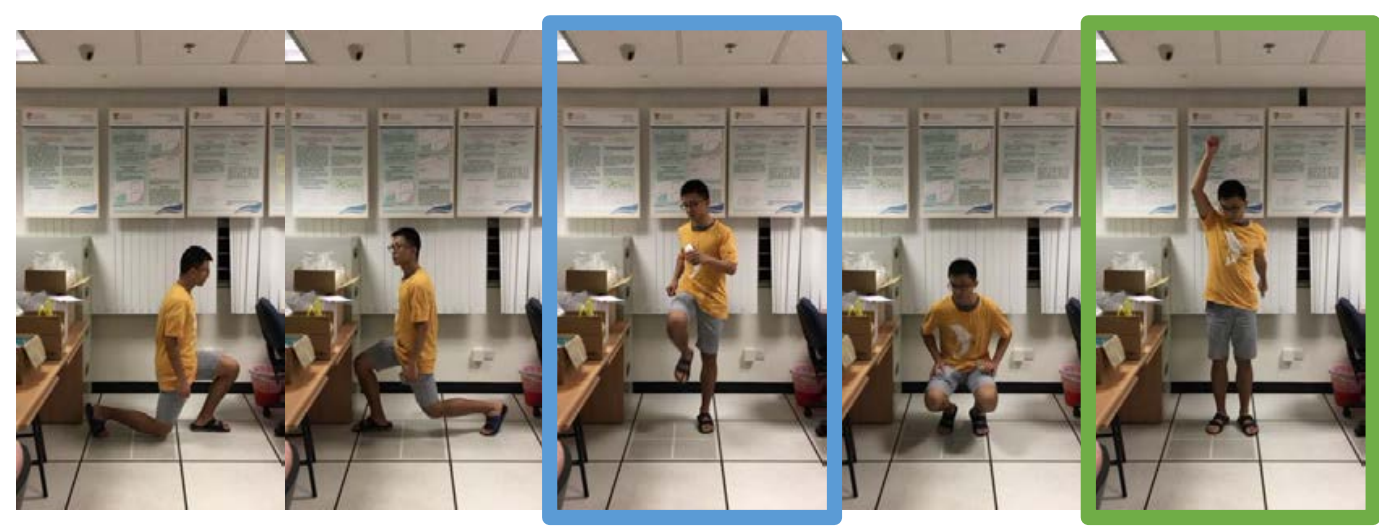

(a) Lower body warm up

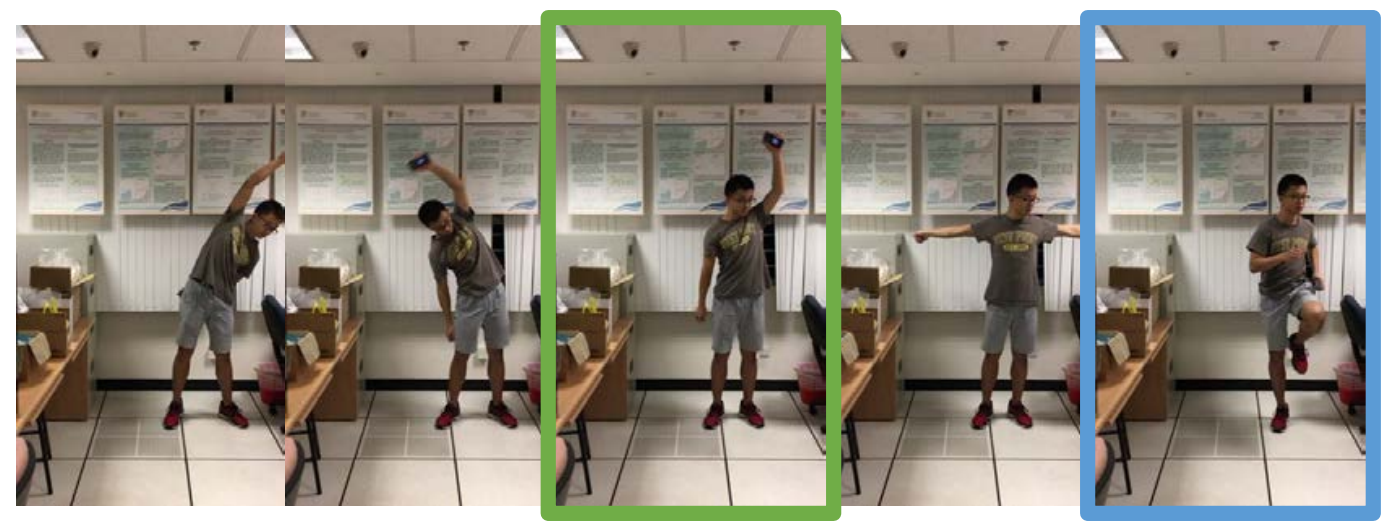

(b) Upper body warm up

Figure 4.4: Some frame examples of WarmUp2 dataset.

to 5. 1023 frame-level descriptors are extracted in total. To avoid over-fitting, we divide the training videos into two parts. The first part consists of two videos for each category, which are used for training the DASD model while the second part including another four videos is used for estimating the rest parameters. In our experiments, the balance parameters $\lambda$ in Eq.(4.34) and $\theta$ in Eq.(4.22) are fixed to $\lambda=0.01, \theta=0.01$.

\section{Baselines}

In our experiments, multiple baseline methods are compared, which are described as follows.

- Independent Results: 
The results obtained from the DASD model in Chapter 3 without any smoothing methods.

- Mean filter:

The results of the DASD model followed by an on-line mean filter, which filters the scores of action states of the current time $t$ by the scores from $[t-L, t] . L$ is the length of the filter which is fixed to $L=4$ in our experiments.

- HMM based classifier:

In HMM, the transition probability only depends on the current action state and the previous one action state while the duration and the progression of the current state are not included. Thus the action state Markov model defined in Eq.(4.19) is simplified to $P\left(s_{\tau} \mid s_{\tau-1}, y_{\tau}\right)$. Thus, HMM based classification model is shown as:

$$
\max _{S_{t}, y_{t}, A_{t}}\left\{\prod_{\tau=1}^{t} P\left(A_{\tau} \mid S_{\tau}, y_{\tau}\right) P\left(S_{\tau} \mid x_{\tau}\right) P\left(s_{\tau} \mid s_{\tau-1}, y_{\tau}\right) P\left(y_{\tau} \mid s_{\tau-1}, y_{\tau-1}\right)\right\} .
$$

- Maximum-Entropy Markov Model (MEMM) based classifier:

MEMM can be used in the on-line scenario where the current label is independent of the future observations [68]. In MEMM, the dependency among the feature and the neighboring action states are modeled together, which is $P\left(s_{\tau} \mid s_{\tau-1}, x_{\tau}, y_{\tau}\right)$. By replacing the action state independent model and the action state Markov model in Eq.(4.19) with the above expression, MEMM based classifier is written as:

$$
\max _{S_{t}, y_{t}, A_{t}}\left\{\prod_{\tau=1}^{t} P\left(A_{\tau} \mid S_{\tau}, y_{\tau}\right) P\left(s_{\tau} \mid s_{\tau-1}, x_{\tau}, y_{\tau}\right) P\left(y_{\tau} \mid s_{\tau-1}, y_{\tau-1}\right)\right\} .
$$

Linear SVM is employed when training the parameters for each action state transition. Note that the inference method for baselines also remains the same as our proposed model. 


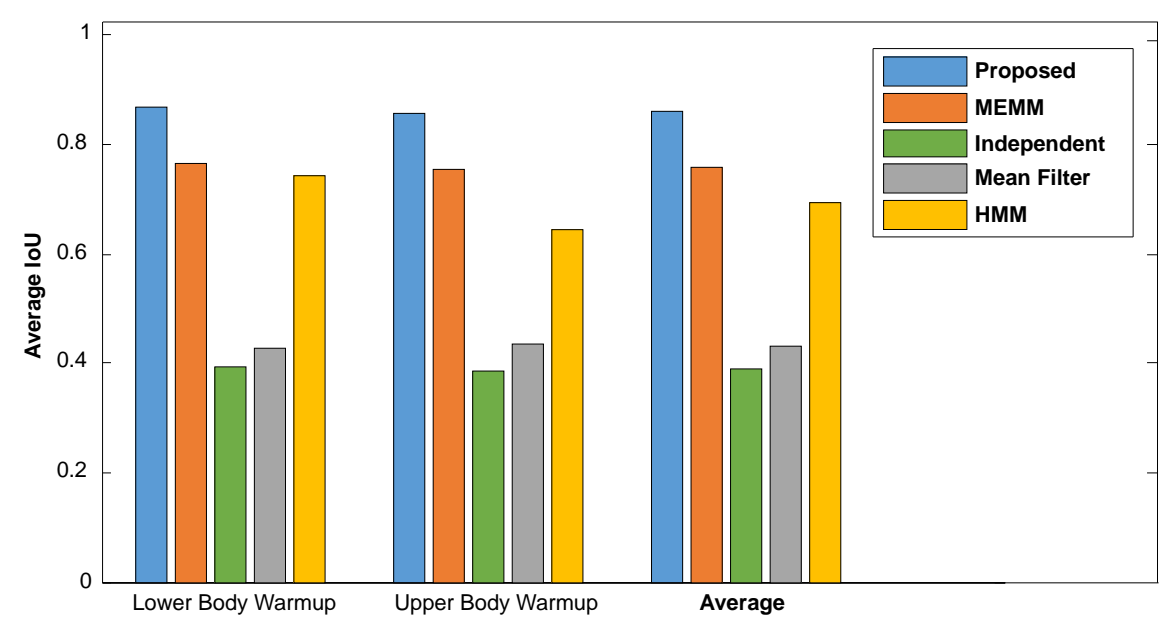

Figure 4.5: Average IoU in activity categories of our approach and baselines.

\subsubsection{Experiment on Action State Segmentation}

In this section, we aim to evaluate the ability to segment the testing videos. To make the best of the dataset, we employ Leave-One-Out (LOO) cross validation scheme, where in each round, one video in each activity category is selected for testing and the rest are used for training. Here we compute the average Intersection over Union (IoU) to represent the precision of the segmentations. Given a testing video after performing the inference and the ground truth of segmentations, which are represented by 5 pairs of starting and ending points of the states $\left[t_{i}^{(s)}, t_{i}^{(e)}\right]_{i=1: 5}$, the estimated action states with $n$ longest length $\left(i . e .\left[\hat{t}_{j}^{(s)}, \hat{t}_{j}^{(e)}\right]_{j=1: n}\right)$ are chosen, where $n=\min \left\{m_{T}, 5\right\}$. The IoU of one video is computed by

$$
\frac{1}{n} \sum_{j=1}^{n} \max _{i=1: 5}\left\{\frac{\left[t_{i}^{(s)}, t_{i}^{(e)}\right] \cap\left[\hat{t}_{j}^{(s)}, \hat{t}_{j}^{(e)}\right]}{\left[t_{i}^{(s)}, t_{i}^{(e)}\right] \cup\left[\hat{t}_{j}^{(s)}, \hat{t}_{j}^{(e)}\right]}\right\}
$$

where the term in the curly brackets is the representation of IoU of two intervals.

Table 4.2: Average IoU of our approach and baselines in the experiment on action states segmentation.

\begin{tabular}{c|ccccc}
\hline Method & Independent & Mean Filter & HMM & MEMM & Proposed \\
\hline Average IoU & 0.390 & 0.430 & 0.695 & 0.760 & $\mathbf{0 . 8 6 3}$ \\
\hline
\end{tabular}



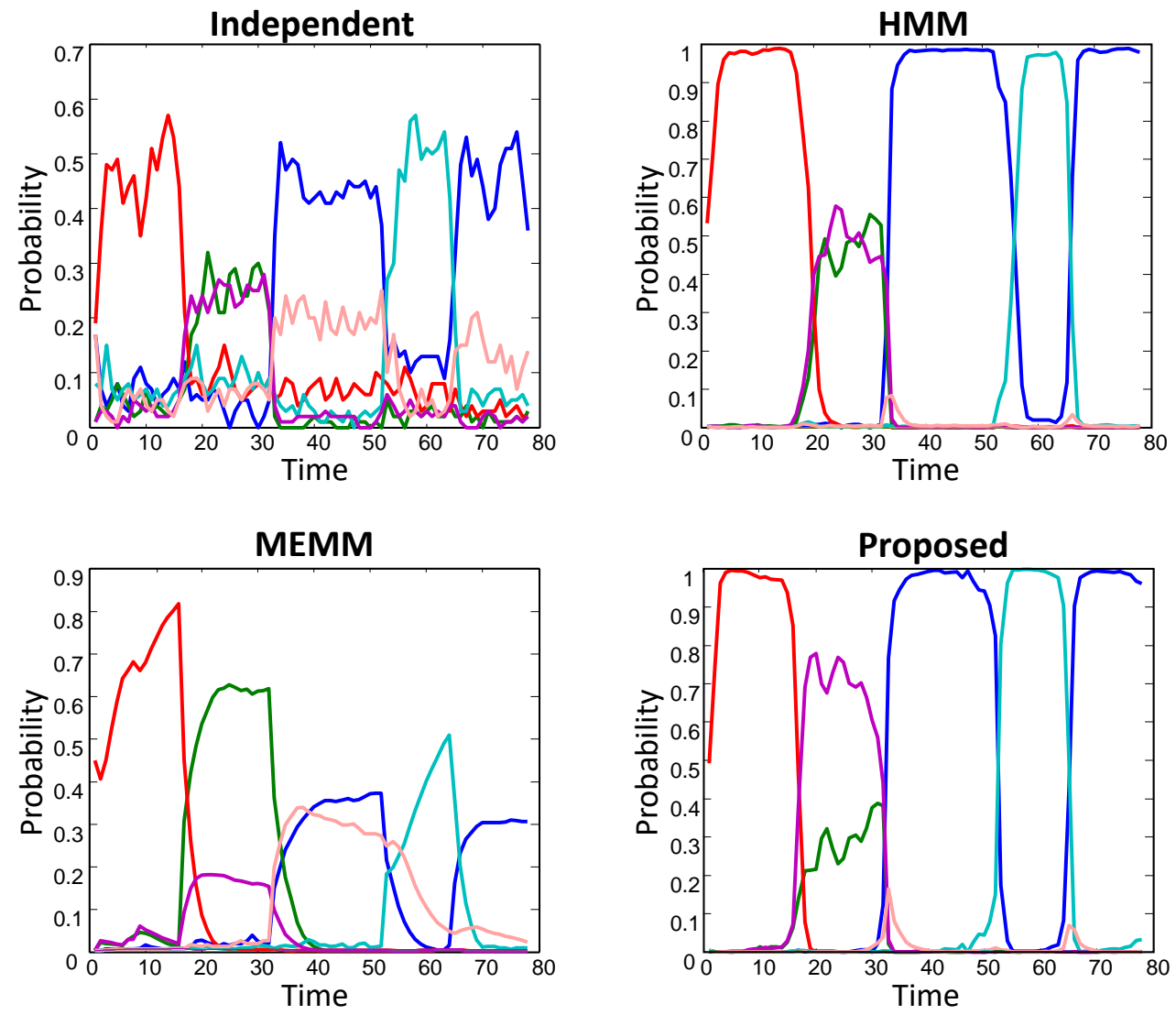

Action State 1

Action State 3

Action State 5

Action State 2

Action State 4

Action State 8

Figure 4.6: An example of the output probabilities of action states by different methods.

The experimental results on the WarmUp2 dataset are shown in Figure 4.5. It can be observed from the results that our approach achieves the best performance among all the methods compared. Table 4.2 shows that by computing the average of two categories, the average IoU of our algorithm is 0.863 , which outperforms MEMM based classifier by 0.103 and HMM based classifier by 0.168 . An example of the result is shown in Figure 4.6, where lines of different colors represent the probabilities of different action states. In this example, action state 2 and 5 are shared action state, action state 1 and 8 are shared state. From the independent results in the top left figure of Figure 4.6 we can see that the probabilities of shared states at one time point are high and similar (i.e. purple line and green line). Our approach performs significantly better at recognizing shared action states and pro- 
viding smoothing scores because that in the action state Markov model, we not only consider the transition between action states, but also model the length of each action states. A high continuation probability $P^{(C)}\left(S_{t}\right)$ ensures that the current action state will not be influenced by shared action states. Differently, HMM based classifier and MEMM based classifier are not capable to capture the duration of the current state. Thus it fails to stick the results to one of the shared states. Furthermore, the diagonal elements in the estimated transition matrix in HMM based classifier is much higher than other elements. Therefore, the transition between action states only occurs when the probability from action state independent model is high enough, which results in a small delay on the transition between neighboring action states.

\subsubsection{Experiment on Activity Recognition}

The performance of activity recognition of our model is testified in this section. Similarly, we also employ LOO cross validation scheme in this experiment. Since our model aims at recognizing activities in the untrimmed video, the frame-level average accuracy is used to represent the performance of different algorithms.

The recognition accuracy of each activity category is illustrated in Figure 4.7 and the mean accuracy of our approach and all the compared baseline methods are listed in Table 4.3. From the Table 4.3, it can be seen that our algorithm successfully recognizes all the temporal windows in the testing videos. The independent results and the mean filter results are not stable where the accuracy of the first

Table 4.3: Mean accuracy of our approach and baselines in the experiment on activity recognition.

\begin{tabular}{c|ccccc}
\hline Method & Independent & Mean Filter & HMM & MEMM & Proposed \\
\hline Mean Accuracy & 0.723 & 0.780 & 0.968 & 0.931 & $\mathbf{1 . 0 0 0}$ \\
\hline
\end{tabular}




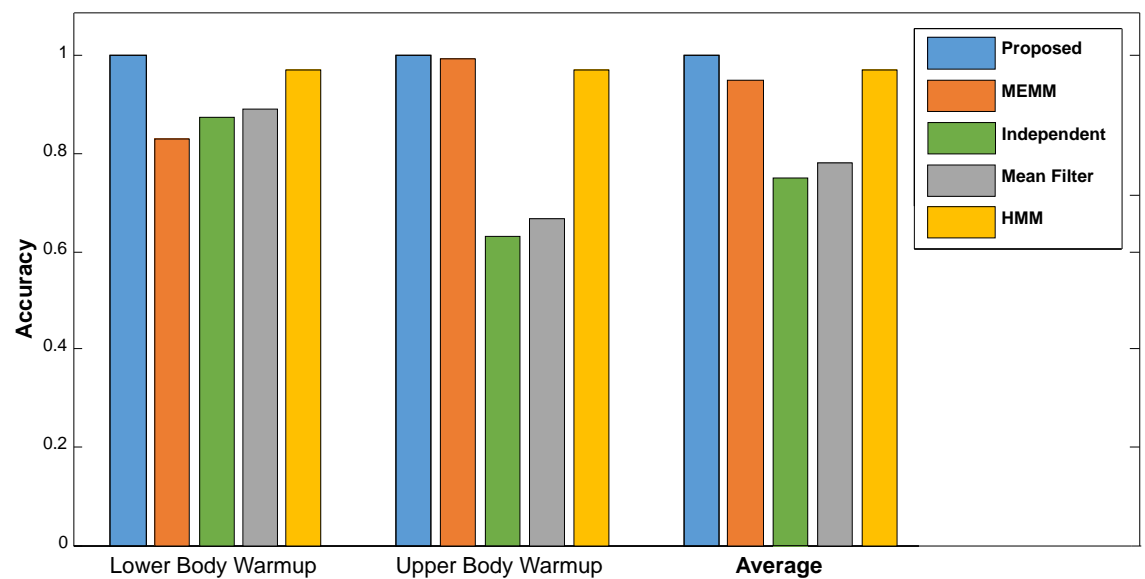

Figure 4.7: Recognition accuracy in activity categories of our approach and the baselines.

category is much higher than that of the second category since the classifier is biased by the training samples of shared action states. The graph based methods such as HMM and MEMM based classifiers do not solve the problem caused by shared states very well either because shared action states weaken the confidence on all the action states, which results in an incorrect classification.

\subsubsection{Experiment on Alarm Generation}

We also evaluate the ability of detecting the abnormal action states in an activity of our algorithm. In this experiment, we train the model by all the 14 videos of WarmUp2 dataset. Another 12 videos are collected in which every four videos contain one of the three types of anomaly. Three different types of anomaly are evaluated separately where temporal-window-level average precision is employed as the metric. The results of baselines are obtained by combining their algorithms with our alarm generation mechanism. Besides the baseline methods mentioned in Section 4.3.1, the Flexible Ordered Graph (FOG) proposed in [48] is also compared. Since the FOG method is not designed to detect overly long or overly short action states, only the performance of alarm for abnormal action states of this method is reported.

The results of this experiment can be seen in Table 4.4. For all the three types 
Table 4.4: Average precision of 3 types of alarms generation.

\begin{tabular}{c|ccc}
\hline Methods & $\begin{array}{c}\text { Alarm for long } \\
\text { duration states }\end{array}$ & $\begin{array}{c}\text { Alarm for short } \\
\text { duration states }\end{array}$ & $\begin{array}{c}\text { Alarm for } \\
\text { abnormal states }\end{array}$ \\
\hline Independent & 0.740 & 0.508 & 0.634 \\
Mean Filter & 0.738 & 0.496 & 0.607 \\
HMM & 0.870 & 0.457 & 0.773 \\
MEMM & 0.712 & 0.497 & 0.687 \\
FOG [48] & - & - & 0.253 \\
Proposed & $\mathbf{0 . 8 8 8}$ & $\mathbf{0 . 8 1 5}$ & $\mathbf{0 . 9 1 9}$ \\
\hline
\end{tabular}

of alarm generation, our approach achieves the best average precision. Figure 4.8 shows the precision of our methods approximately equals to 1 when the recall equals to 0.88 , which is significantly better than other methods. Although MEMM based classifier shows good performance in action states segmentation and activity recognition, it is not able to generate the alarms for abnormal action states. Since there are no training samples for abnormal action states, the jointly learned probabilities $P\left(s_{t} \mid s_{t-1}, y_{t}, x_{t}\right)$ in MEMM based classifier will limit the transitions to the abnormal action states. On the contrary, the probability $P\left(s_{t} \mid x_{t}\right)$ in our

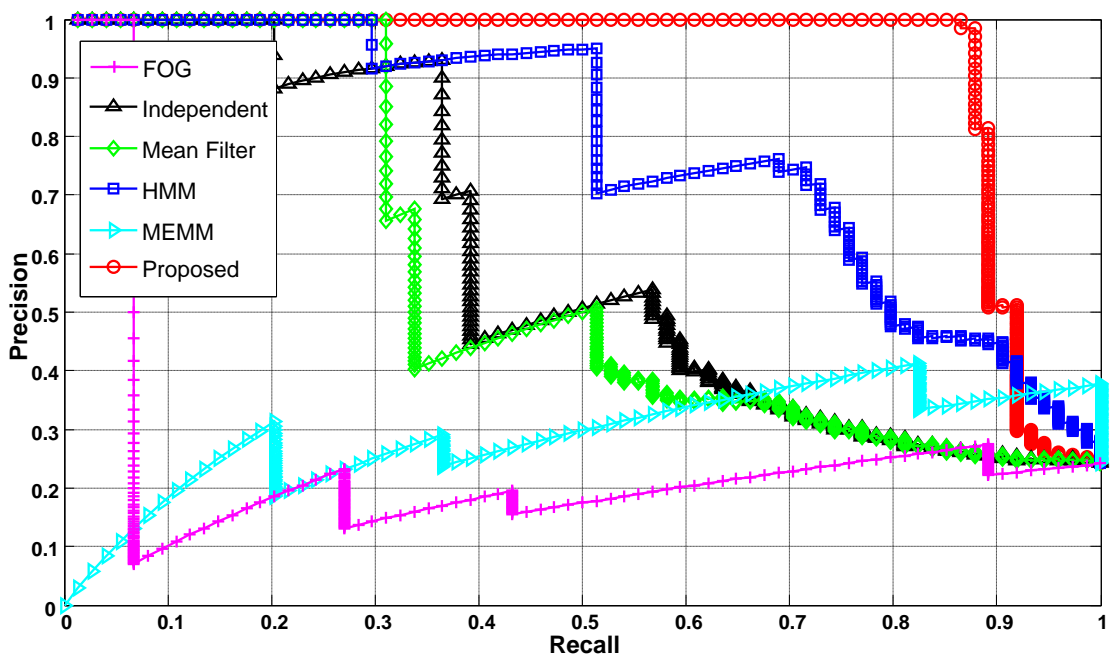

Figure 4.8: The curve of precision and recall on alarm generation for abnormal action states by different methods. 
algorithm ensures the action states will be recognized correctly even when it is an abnormal action state in the activity. The Flexible Ordered Graph misses most of the alarms because this method detects the abnormal states only based on the transition between action states without involving the progression of the activity.

\subsection{Concluding Remarks}

In this chapter, we introduce the Statistical Activity Model for on-line activity recognition and anomaly detection. In this model, the temporal dependencies between action states and within each action state are captured, which is used for parsing the positions of action states and activities. To generate alarms for anomalies, the proposed model integrates a novel alarm generation mechanism which is based on the transition between action states as well as the progression of the activity. The experimental results show that our approach outperforms the baseline methods and state-of-the-art method on the on-line task of action states segmentation, activity recognition, and alarm generation. 


\section{Chapter 5}

\section{Conclusions and Future Work}

\subsection{Conclusions}

In this thesis, a systematic study on challenging tasks of on-line human action and activity recognition has been presented, which is summarized in the following.

Firstly, the multi-assignment BoW representation is introduced, which is the improvement of standard BoW representation by assigning each local feature vector to multiple codes in the trained codebook instead of one. It is evaluated by the task of on-line action recognition and the experimental results show that noises from frame-level descriptors can be suppressed and the mean accuracy of recognition is improved by multi-assignment BoW representation. To solve the problem of large intra-class variation, which caused by the partial observation of the video, in on-line action recognition, the Discriminative Action States Discovery (DASD) approach is proposed, which aims at discovering the most discriminative action states for each action while training the classifiers. By employing our algorithm, distributions of different action states within every action category are learned and the variation within each action state is minimized. Experimental results demonstrate that our method has the superior performance against the baseline method and the state-of-the-art methods. Besides on-line action recognition, DASD can also solve action prediction problem, especially when the observation of the video is extremely limited. 
Secondly, the Statistical Activity Model (SAM) is introduced for joint on-line activity recognition and anomaly detection. In this model, the temporal dependencies between action states in an activity are captured. Different from other graph based models, we consider the transition between different action states as well as the length of each action states, which solves the problems caused by shared action states in multiple activities and improves the ability to parse positions of actions and activities in the untrimmed video. Three different experiments are designed to evaluate the performance of our model on the task of on-line action states segmentation, activity recognition and alarm generation respectively. The results prove that our algorithm outperforms the baseline methods and the state-of-the-art method.

Last but not the least, the most time-saving local features (i.e. DTF and C3D), middle-level representation (i.e. multi-assignment BoW), and classifiers (i.e. linear classifiers and random forest) are employed in our framework, which ensure the real-time processing speed of the on-line activity recognition system built by our algorithms.

To summarize, this research work addressed the main challenges in the task of on-line action and activity recognition as mentioned above. Several effective and efficient algorithms are developed, which show promising results on several action and activity datasets. Additionally, the algorithms are employed to build an online action and activity recognition system which can recognize human action and activity at the real-time speed.

\subsection{Future Work}

Some possible future research aspects are listed as follows: real-world testing on Statistical Activity Model, adaptive action states discovery to determine the optimized number of action states, and on-line multi-person activity recognition in 
untrimmed videos.

\subsubsection{Real-world Testing}

Firstly, our Statistical Activity Model will be evaluated by more complicated activities of real-world scenario. We will work on collecting more data of daily life activities which contain much more variance. For the full body activities, for example, training soldiers of S.W.A.T. ${ }^{1}$ to clear the room, normal hand-held cameras or surveillance cameras are required to collect the videos, while for the activities which are mostly completed by hands such as cooking and assembling, we need egocentric cameras $[48,69]$ to build egocentric vision videos.

\subsubsection{Adaptive Action States Discovery}

As discussed in Chapter 4, the proposed DASD can learn different distributions of frame-level descriptors of one action category. The results in Section 4.3 show that for different action categories, the best performances are achieved by different number of action states. However, for DASD algorithm, the numbers of action states can only be pre-defined or obtained from cross validation. Therefore, in the future, we will work on how to automatically determine the optimal number of action states as well as learn the patterns for each action state.

\subsubsection{On-line Multi-person Action and Activity Recogni- tion}

In the real-world surveillance scenario, the videos always include multiple persons with different actions instead of focusing on one person. Thus the spatial-temporal action localization is required to detect the position of the performers. There are two main strategies to obtain the positions of actions in video. The first is

\footnotetext{
${ }^{1}$ S.W.A.T. (Special Weapons And Tactics) is a term for law enforcement units which use specialized or military equipment and tactics to deal with incidents beyond the capabilities of normal patrol officers such as hostage situations, high risk warrants and riot control.
} 
employing the spatial-temporal sliding windows of different size, which scan the video on three dimensions [70-73]. However, the scanning stage of these method is extremely time consuming. Another solution is action proposals [74-76], which show good performance and efficiency in recent years. To recognize multiple actions simultaneously, action proposals should be first generated in an on-line processing, followed by recognition stage for each extracted action proposal. 


\section{List of Publications}

[1] Bo Hu, Junsong Yuan, and Yuwei Wu "Discriminative Action States Discovery for On-line Action Recognition," in IEEE Signal Processing Letter (SPL), 2016. 



\section{Bibliography}

[1] D. Tran, L. Bourdev, R. Fergus, L. Torresani, and M. Paluri, "Learning spatiotemporal features with $3 \mathrm{~d}$ convolutional networks," in IEEE international Conference on Computer Vision (ICCV), 2015.

[2] H. Izadinia and M. Shah, "Recognizing complex events using large margin joint low-level event model," in European Conference on Computer Vision (ECCV). Springer, 2012, pp. 430-444.

[3] I. Kviatkovsky, E. Rivlin, and I. Shimshoni, "Online action recognition using covariance of shape and motion," Computer Vision and Image Understanding (CVIU), vol. 129, pp. 15-26, 2014.

[4] C. Lu, J. Shi, and J. Jia, "Abnormal event detection at 150 fps in matlab," in IEEE international Conference on Computer Vision (ICCV), 2013, pp. $2720-2727$.

[5] Y. Cong, J. Yuan, and J. Liu, "Abnormal event detection in crowded scenes using sparse representation," Pattern Recognition (PR), vol. 46, no. 7, pp. 1851-1864, 2013.

[6] R. R. Murphy, T. Nomura, A. Billard, and J. L. Burke, "Human-robot interaction," IEEE Robotics \&5 Automation Magazine, vol. 17, no. 2, pp. 85-89, 2010. 
[7] H. Wang, A. Klaser, C. Schmid, and C.-L. Liu, "Action recognition by dense trajectories," in IEEE Conference on Computer Vision and Pattern Recognition (CVPR), 2011, pp. 3169-3176.

[8] H. Wang and C. Schmid, "Action recognition with improved trajectories," in IEEE international Conference on Computer Vision (ICCV), 2013, pp. 35513558.

[9] H. Wang, A. Kläser, C. Schmid, and C.-L. Liu, "Dense trajectories and motion boundary descriptors for action recognition," International Journal of Computer Vision (IJCV), vol. 103, no. 1, pp. 60-79, 2013.

[10] I. Laptev, "On space-time interest points," International Journal of Computer Vision (IJCV), vol. 64, no. 2-3, pp. 107-123, 2005.

[11] G. Willems, T. Tuytelaars, and L. Van Gool, "An efficient dense and scaleinvariant spatio-temporal interest point detector," in European Conference on Computer Vision (ECCV). Springer, 2008, pp. 650-663.

[12] P. Dollár, V. Rabaud, G. Cottrell, and S. Belongie, "Behavior recognition via sparse spatio-temporal features," in Joint IEEE International Workshop on Visual Surveillance and Performance Evaluation of Tracking and Surveillance. IEEE, 2005, pp. 65-72.

[13] N. Dalal and B. Triggs, "Histograms of oriented gradients for human detection," in IEEE Conference on Computer Vision and Pattern Recognition (CVPR), 2005, pp. 886-893.

[14] N. Dalal, B. Triggs, and C. Schmid, "Human detection using oriented histograms of flow and appearance," in European Conference on Computer Vision (ECCV). Springer, 2006, pp. 428-441. 
[15] J. Sivic and A. Zisserman, "Video google: A text retrieval approach to object matching in videos," in IEEE international Conference on Computer Vision (ICCV), 2003, pp. 1470-1477.

[16] F. Perronnin and C. Dance, "Fisher kernels on visual vocabularies for image categorization," in IEEE Conference on Computer Vision and Pattern Recognition (CVPR), 2007, pp. 1-8.

[17] F. Perronnin, J. Sánchez, and T. Mensink, "Improving the fisher kernel for large-scale image classification," in European Conference on Computer Vision (ECCV). Springer, 2010, pp. 143-156.

[18] T. S. Jaakkola, D. Haussler et al., "Exploiting generative models in discriminative classifiers," in Conference on Neural Information Processing Systems (NIPS), 1999, pp. 487-493.

[19] H. Jégou, M. Douze, C. Schmid, and P. Pérez, "Aggregating local descriptors into a compact image representation," in IEEE Conference on Computer Vision and Pattern Recognition (CVPR), 2010, pp. 3304-3311.

[20] S. Sadanand and J. J. Corso, "Action bank: A high-level representation of activity in video," in IEEE Conference on Computer Vision and Pattern Recognition (CVPR), 2012, pp. 1234-1241.

[21] M. Raptis, I. Kokkinos, and S. Soatto, "Discovering discriminative action parts from mid-level video representations," in IEEE Conference on Computer Vision and Pattern Recognition (CVPR), 2012, pp. 1242-1249.

[22] A. Jain, A. Gupta, M. Rodriguez, and L. Davis, "Representing videos using mid-level discriminative patches," in IEEE Conference on Computer Vision and Pattern Recognition (CVPR), 2013, pp. 2571-2578. 
[23] S. Narayan and K. R. Ramakrishnan, "A cause and effect analysis of motion trajectories for modeling actions," in IEEE Conference on Computer Vision and Pattern Recognition (CVPR), 2014, pp. 2633-2640.

[24] B. Fernando, E. Gavves, J. Oramas, A. Ghodrati, and T. Tuytelaars, "Modeling video evolution for action recognition," in IEEE Conference on Computer Vision and Pattern Recognition (CVPR), 2015, pp. 5378-5387.

[25] S. Ma, L. Sigal, and S. Sclaroff, "Space-time tree ensemble for action recognition," in IEEE Conference on Computer Vision and Pattern Recognition (CVPR), 2015, pp. 5024-5032.

[26] P. Bilinski and F. Bremond, "Video covariance matrix logarithm for human action recognition in videos," in International Joint Conference on Artificial Intelligence (IJCAI), 2015.

[27] Y. Jia, E. Shelhamer, J. Donahue, S. Karayev, J. Long, R. Girshick, S. Guadarrama, and T. Darrell, "Caffe: Convolutional architecture for fast feature embedding," in ACM International Conference on Multimedia (MM). ACM, 2014, pp. 675-678.

[28] A. Krizhevsky, I. Sutskever, and G. E. Hinton, "Imagenet classification with deep convolutional neural networks," in Conference on Neural Information Processing Systems (NIPS), 2012, pp. 1097-1105.

[29] K. Simonyan and A. Zisserman, "Two-stream convolutional networks for action recognition in videos," in Conference on Neural Information Processing Systems (NIPS), Z. Ghahramani, M. Welling, C. Cortes, N. D. Lawrence, and K. Q. Weinberger, Eds., 2014, pp. 568-576.

[30] J. Yue-Hei Ng, M. Hausknecht, S. Vijayanarasimhan, O. Vinyals, R. Monga, and G. Toderici, "Beyond short snippets: Deep networks for video classifi- 
cation," in IEEE Conference on Computer Vision and Pattern Recognition (CVPR), 2015, pp. 1-8.

[31] W. Zhu, J. Hu, G. Sun, X. Cao, and Y. Qiao, "A key volume mining deep framework for action recognition," in IEEE Conference on Computer Vision and Pattern Recognition (CVPR), June 2016.

[32] H. Bilen, B. Fernando, E. Gavves, A. Vedaldi, and S. Gould, "Dynamic image networks for action recognition," in IEEE Conference on Computer Vision and Pattern Recognition (CVPR), June 2016.

[33] C. Feichtenhofer, A. Pinz, and A. Zisserman, "Convolutional two-stream network fusion for video action recognition," in IEEE Conference on Computer Vision and Pattern Recognition (CVPR), June 2016.

[34] A. Karpathy, G. Toderici, S. Shetty, T. Leung, R. Sukthankar, and L. Fei-Fei, "Large-scale video classification with convolutional neural networks," in IEEE Conference on Computer Vision and Pattern Recognition (CVPR), 2014, pp. $1725-1732$.

[35] A. Yao, J. Gall, and L. Van Gool, "A hough transform-based voting framework for action recognition," in IEEE Conference on Computer Vision and Pattern Recognition (CVPR), 2010, pp. 2061-2068.

[36] G. Yu, J. Yuan, and Z. Liu, "Propagative hough voting for human activity detection and recognition," IEEE Transactions on Circuits and Systems for Video Technology (T-CSVT), vol. 25, no. 1, pp. 87-98, 2015.

[37] M. Jain, H. Jégou, and P. Bouthemy, "Better exploiting motion for better action recognition," in IEEE Conference on Computer Vision and Pattern Recognition (CVPR), 2013, pp. 2555-2562. 
[38] K.-T. Lai, F. X. Yu, M.-S. Chen, and S.-F. Chang, "Video event detection by inferring temporal instance labels," in IEEE Conference on Computer Vision and Pattern Recognition (CVPR), 2014, pp. 2251-2258.

[39] W. Sultani and I. Saleemi, "Human action recognition across datasets by foreground-weighted histogram decomposition," in Proc. IEEE Conf. on Computer Vision and Pattern Recognition (CVPR). IEEE, 2014, pp. 764-771.

[40] M. Jain, J. C. van Gemert, and C. G. Snoek, "What do 15,000 object categories tell us about classifying and localizing actions?" in IEEE Conference on Computer Vision and Pattern Recognition (CVPR), 2015, pp. 46-55.

[41] Y. Song, Y.-T. Zheng, S. Tang, X. Zhou, Y. Zhang, S. Lin, and T.-S. Chua, "Localized multiple kernel learning for realistic human action recognition in videos," IEEE Transactions on Circuits and Systems for Video Technology (T-CSVT), vol. 21, no. 9, pp. 1193-1202, 2011.

[42] Y. Song, S. Liu, and J. Tang, "Describing trajectory of surface patch for human action recognition on rgb and depth videos," IEEE Signal Processing Letters (SPL), vol. 22, no. 4, pp. 426-429, 2015.

[43] Y. Li, W. Li, V. Mahadevan, and N. Vasconcelos, "Vlad3: Encoding dynamics of deep features for action recognition," in IEEE Conference on Computer Vision and Pattern Recognition (CVPR), June 2016.

[44] C. Ellis, S. Z. Masood, M. F. Tappen, J. J. Laviola Jr, and R. Sukthankar, "Exploring the trade-off between accuracy and observational latency in action recognition," International Journal of Computer Vision (IJCV), vol. 101, no. 3, pp. 420-436, 2013.

[45] G. Yu, Z. Liu, and J. Yuan, "Discriminative orderlet mining for real-time recognition of human-object interaction," in Asian Conference on Computer Vision (ACCV), 2014. 
[46] M. Albanese, R. Chellappa, N. Cuntoor, V. Moscato, A. Picariello, V. Subrahmanian, and O. Udrea, "Pads: A probabilistic activity detection framework for video data," IEEE Transactions on Pattern Analysis and Machine Intelligence (T-PAMI), vol. 32, no. 12, pp. 2246-2261, 2010.

[47] K. Schindler and L. Van Gool, "Action snippets: How many frames does human action recognition require?" in IEEE Conference on Computer Vision and Pattern Recognition (CVPR), 2008, pp. 1-8.

[48] B. Soran, A. Farhadi, and L. Shapiro, "Generating notifications for missing actions: Don't forget to turn the lights off!" in Proceedings of the IEEE International Conference on Computer Vision, 2015, pp. 4669-4677.

[49] S. R. Fanello, I. Gori, G. Metta, and F. Odone, "Keep it simple and sparse: Real-time action recognition," The Journal of Machine Learning Research (JMLR), vol. 14, no. 1, pp. 2617-2640, 2013.

[50] M. Ryoo, "Human activity prediction: Early recognition of ongoing activities from streaming videos," in IEEE international Conference on Computer Vision (ICCV), 2011, pp. 1036-1043.

[51] Y. Cao, D. Barrett, A. Barbu, S. Narayanaswamy, H. Yu, A. Michaux, Y. Lin, S. Dickinson, J. Siskind, and S. Wang, "Recognize human activities from partially observed videos," in IEEE Conference on Computer Vision and Pattern Recognition (CVPR), 2013, pp. 2658-2665.

[52] M. Hoai and F. De la Torre, "Max-margin early event detectors," International Journal of Computer Vision (IJCV), vol. 107, no. 2, pp. 191-202, 2014.

[53] G. Yu, J. Yuan, and Z. Liu, "Predicting human activities using spatiotemporal structure of interest points," in ACM International Conference on Multimedia (MM), 2012, pp. 1049-1052. 
[54] M. Brand and V. Kettnaker, "Discovery and segmentation of activities in video," IEEE Transactions on Pattern Analysis and Machine Intelligence (TPAMI), vol. 22, no. 8, pp. 844-851, 2000.

[55] E. Fox, E. B. Sudderth, M. I. Jordan, and A. S. Willsky, "Bayesian nonparametric inference of switching dynamic linear models," IEEE Transactions on Signal Processing (T-SP), vol. 59, no. 4, pp. 1569-1585, 2011.

[56] D. L. Vail, M. M. Veloso, and J. D. Lafferty, "Conditional random fields for activity recognition," in International joint conference on Autonomous agents and multiagent systems. ACM, 2007, p. 235.

[57] C. Sun and R. Nevatia, "Active: Activity concept transitions in video event classification," in IEEE Conference on Computer Vision and Pattern Recognition (CVPR), 2013, pp. 913-920.

[58] J. C. Niebles, C.-W. Chen, and L. Fei-Fei, "Modeling temporal structure of decomposable motion segments for activity classification," in European Conference on Computer Vision (ECCV). Springer, 2010, pp. 392-405.

[59] A. Richard and J. Gall, "Temporal action detection using a statistical language model," in IEEE Conference on Computer Vision and Pattern Recognition (CVPR), June 2016.

[60] A. Gupta, P. Srinivasan, J. Shi, and L. S. Davis, "Understanding videos, constructing plots learning a visually grounded storyline model from annotated videos," in IEEE Conference on Computer Vision and Pattern Recognition (CVPR), 2009, pp. 2012-2019.

[61] Y. Cheng, Q. Fan, S. Pankanti, and A. Choudhary, "Temporal sequence modeling for video event detection," in IEEE Conference on Computer Vision and Pattern Recognition (CVPR), 2014, pp. 2227-2234. 
[62] Y. Shi, Y. Huang, D. Minnen, A. Bobick, and I. Essa, "Propagation networks for recognition of partially ordered sequential action," in IEEE Conference on Computer Vision and Pattern Recognition (CVPR), 2004, pp. 1-8.

[63] S. Bhattacharya, M. M. Kalayeh, R. Sukthankar, and M. Shah, "Recognition of complex events: Exploiting temporal dynamics between underlying concepts," in IEEE Conference on Computer Vision and Pattern Recognition (CVPR), 2014, pp. 2235-2242.

[64] M. Hoai and A. Zisserman, "Discriminative sub-categorization," in IEEE Conference on Computer Vision and Pattern Recognition (CVPR), 2013, pp. $1666-1673$.

[65] R.-E. Fan, K.-W. Chang, C.-J. Hsieh, X.-R. Wang, and C.-J. Lin, "Liblinear: A library for large linear classification," The Journal of Machine Learning Research (JMLR), vol. 9, pp. 1871-1874, 2008.

[66] K. Soomro, A. R. Zamir, and M. Shah, "Ucf101: A dataset of 101 human actions classes from videos in the wild," arXiv preprint arXiv:1212.0402, 2012.

[67] M. S. Ryoo and J. Aggarwal, "Ut-interaction dataset, icpr contest on semantic description of human activities (sdha)," in IEEE International Conference on Pattern Recognition (ICPR) Workshops, 2010.

[68] A. McCallum, D. Freitag, and F. C. Pereira, "Maximum entropy markov models for information extraction and segmentation." in International Conference on Machine Learning (ICML), vol. 17, 2000, pp. 591-598.

[69] Y.-C. Su and K. Grauman, "Detecting engagement in egocentric video," in European Conference on Computer Vision (ECCV). Springer, 2016.

[70] J. Yuan, Z. Liu, and Y. Wu, "Discriminative video pattern search for efficient action detection," IEEE Transactions on Pattern Analysis and Machine Intelligence (T-PAMI), vol. 33, no. 9, pp. 1728-1743, 2011. 
[71] Y. Ke, R. Sukthankar, and M. Hebert, "Event detection in crowded videos," in International Conference on Computer Vision (ICCV). IEEE, 2007.

[72] P. Siva and T. Xiang, "Weakly supervised action detection." in British Machine Vision Conference (BMVC), 2011.

[73] D. Tran and J. Yuan, "Max-margin structured output regression for spatiotemporal action localization," in Conference on Neural Information Processing Systems (NIPS), 2012, pp. 350-358.

[74] G. Yu and J. Yuan, "Fast action proposals for human action detection and search," in IEEE Conference on Computer Vision and Pattern Recognition (CVPR), 2015, pp. 1302-1311.

[75] P. Weinzaepfel, Z. Harchaoui, and C. Schmid, "Learning to track for spatiotemporal action localization," in IEEE International Conference on Computer Vision (ICCV), 2015, pp. 3164-3172.

[76] J. van Gemert, M. Jain, E. Gati, and C. Snoek, "Apt: Action localization proposals from dense trajectories," in British Machine Vision Conference (BMVC), 2015. 